\title{
TRANSCENDENTAL JULIA SETS WITH FRACTIONAL PACKING DIMENSION
}

\author{
JACK BURKART
}

\begin{abstract}
We construct transcendental entire functions whose Julia sets have packing dimension in $(1,2)$. These are the first examples where the computed packing dimension is not 1 or 2 . Our analysis will allow us further show that the set of packing dimensions attained is dense in the interval $(1,2)$, and that the Hausdorff dimension of the Julia sets can be made arbitrarily close to the corresponding packing dimension.
\end{abstract}

\section{INTRODUCTION}

Let $f: \mathbb{C} \rightarrow \mathbb{C}$ be a transcendental (non-polynomial) entire function. We denote the $n$th iterate of $f$ by $f^{n}$. We define the Fatou set, $\mathcal{F}(f)$, to be the set of all points so that $\left\{f^{n}\right\}_{n=1}^{\infty}$ locally forms a normal family. Thus the Fatou set is the "stable" set for the dynamics of $f$. We define the Julia set, $\mathcal{J}(f)$, to be the complement of the Fatou set. This is the set where the dynamics of $f$ are chaotic. We refer the reader to [CG93] and Sch10] for an introduction to complex dynamics in the rational and transcendental setting, respectively.

One of the goals of complex dynamics is to understand the geometric and topological properties of the Julia set. In this paper we prove Theorem 1.1.

Theorem 1.1. There exists a transcendental entire function $f: \mathbb{C} \rightarrow \mathbb{C}$ such that the packing dimension of $\mathcal{J}(f)$ belongs to $(1,2)$.

Stallard asked in Sta08] if there exists a transcendental meromorphic (we consider entire functions as a special case of meromorphic functions) function for which the packing and Hausdorff dimensions of the Julia set are not integers and equal. Our techniques generate a family of entire functions, and we will actually prove the following stronger result which offers positive progress towards the construction of such a function. We denote the Hausdorff and packing dimensions (defined in Section 4) of a set $K$ by $\operatorname{dim}_{H}(K)$ and $\operatorname{dim}_{P}(K)$, respectively.

Theorem 1.2. Given $s \in(1,2)$ and $\epsilon_{0}>0$, there exists a transcendental entire $f$ so that

$$
s-\epsilon_{0} \leq \operatorname{dim}_{\mathrm{H}}(\mathcal{J}(f)) \leq \operatorname{dim}_{\mathrm{P}}(\mathcal{J}(f)) \leq s+\epsilon_{0} .
$$

In particular, the set of packing dimensions attained by Julia sets of transcendental entire functions is dense in $(1,2)$.

In Bak75], Baker proved that the Julia set of a transcendental entire function must always contain a non-trivial, compact, connected set, and it follows immediately that the Hausdorff dimension of the Julia set must always be greater than 2021.

Received by the editors August 26, 2019, and, in revised form, July 8, 2020, and March 9,

2020 Mathematics Subject Classification. Primary 37C45, 37F10. 
or equal to 1. In Mis81, Misiurewicz showed that the Julia set of $\exp (z)$ was the entire complex plane, and in McM87. McMullen showed that the Julia sets of the exponential and sine families of entire functions always have Hausdorff dimension 2 , but need not be all of $\mathbb{C}$. These examples can also have positive or zero area measure. Reducing the dimension of the Julia set is therefore the difficult task in the transcendental setting, and in Sta91, Stallard constructed examples in the Eremenko-Lyubich class that had Hausdorff dimension arbitrarily close to 1, and refined this result further in [Sta97] and Sta00] to include all values in $(1,2)$. Moreover, in Sta96], Stallard showed that in the Eremenko-Lyubich class the Hausdorff dimension must be strictly greater than 1. Recently, in Bis18, Bishop constructed a transcendental entire function with Julia set having Hausdorff dimension 1. This example demonstrates that all values of Hausdorff dimension in $[1,2]$ can be achieved.

Less is known about the packing dimension in the transcendental setting. In RS05, Rippon and Stallard show that if $f$ belongs to the Eremenko-Lyubich class, then the packing dimension of the Julia set of $f$ is 2. Bishop computed the packing dimension of the Julia set of his example above to be 1 . Our examples are the first of their kind where the computed packing dimension is strictly between 1 and 2. Packing dimension and other various dimensions relevant to the paper are defined in Section 4. Figure 1 summarizes what has been proven about the possible Hausdorff and packing dimension pairs attained by Julia sets of transcendental entire functions.

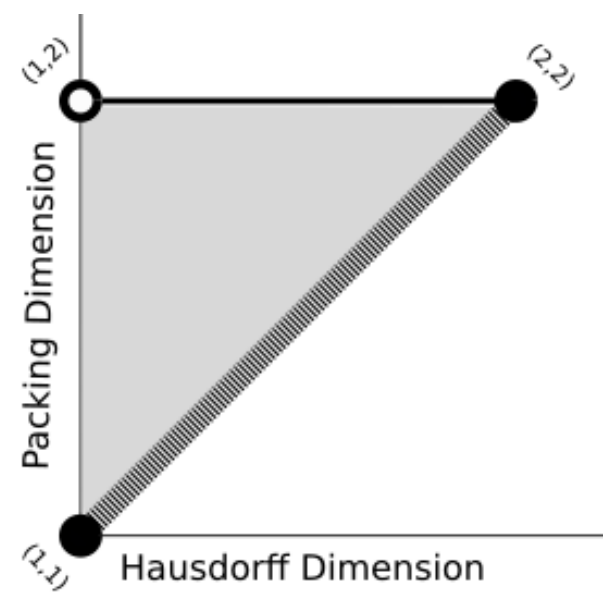

FIgURE 1. A graphic showing the possible and attained Hausdorff and packing dimension pairs for transcendental entire functions. All possible pairs are shaded in light gray, and all attained values are colored black. The point $(2,2)$ is attained by families of the exponential and sine functions. The upper segment is due to the work of Stallard, and the point $(1,1)$ is due to Bishop. Our contribution uses enlarged, dashed lines, to emphasize that a dense set of dimensions is attained very close but perhaps not on the diagonal. 
We would like to point out how our construction differs from the constructions cited above. Since Stallard's examples belong to the Eremenko-Lyubich class, the packing dimension of those Julia sets must be 2, even though the Hausdorff dimension can attain any value in $(1,2)$. In our examples, the packing and Hausdorff dimensions may be arranged to be arbitrarily close. The dynamical behavior of our examples is also much different; the functions we construct have multiply connected Fatou components which do not occur in the Eremenko-Lyubich class. Stallard uses a family of functions defined via a Cauchy integral, whereas we use an infinite product construction similar to Bishop. Our examples look very similar to Bishop's at first glance but there are many major differences. The most obvious difference is the dynamical behavior near the origin; our examples have an attracting basin with quasicircle boundary near the origin, whereas Bishop's contains a Cantor repeller. This difference introduces what we call 'wiggly' Fatou components, which we describe in Section 2. Another difference is that the Hausdorff and packing dimensions in Bishop's example are supported on the boundaries of Fatou components which escape quickly, and the dynamics are simple on these boundaries. In our examples, we will see that the dimension of the Julia set is supported on buried points that are not on the boundary of any Fatou component. In Bishop's example, the buried points have dimension close to zero. The dynamics on the buried points are more intricate; the buried points contain bounded orbit points, escaping points, and so-called bungee orbits; see Section 10.

\section{Outline of the PROOF}

We will construct a function $f: \mathbb{C} \rightarrow \mathbb{C}$ depending on parameters $N \in \mathbb{N}, R \in \mathbb{R}$, and $c$ in the main cardioid of the Mandelbrot set. Define $g(z)=z^{2}+c$. The function $f$ will be $g$ iterated $N$ times multiplied by an infinite product. As a formula,

$$
f(z)=g^{N}(z) \cdot \prod_{k=1}^{\infty}\left(1-\frac{1}{2}\left(\frac{z}{R_{k}}\right)^{n_{k}}\right)=g^{N}(z)(1+\epsilon(z)) .
$$

Here, $n_{k}=2^{N+k-1}$, and the sequence $\left\{R_{k}\right\}_{k=1}^{\infty}$ grows super-exponentially and is defined inductively starting from a large initial parameter $R$. The choices are made so that near the origin, the infinite product can be made uniformly close to the constant function 1 . We will sometimes write the infinite product as $(1+\epsilon(z))$ to emphasize this fact, where $\epsilon(z)$ is a holomorphic function uniformly close to 0 on a large neighborhood of the origin.

In Section 3 we discuss the facts we will need about conformal, quasiconformal, and polynomial-like mappings. In Section 4 we define and discuss what we call Whitney-type decompositions, a natural generalization of Whitney decompositions composed of dyadic squares and our main tool for calculating the packing dimension. In Section 5, we will carefully define $f$ and show it defines an entire function. In Section 6 we decompose a region of the plane far from the origin into alternating annuli $A_{k}$ and $B_{k}$, where the modulus of $A_{k}$ is fixed and the modulus of $B_{k}$ increases as $k \rightarrow \infty$. We will show that $f\left(B_{k}\right) \subset B_{k+1}$, and that if a point ever lands iterates into $B_{k}$, it diverges locally uniformly to $\infty$ under $f$. The existence of these "absorbing" annuli $B_{k}$ of increasing modulus is always true for functions with multiply connected wandering domains; see p.25 in [Zhe06] and also BRS13. for this and related results. In our example, we will additionally show that on $B_{k}$, $f$ is a small perturbation of a constant multiple of the monomial $z^{2 n_{k}}$. Therefore, 
all the interesting dynamical behavior happens in the annuli $A_{k}$. We will show that $A_{k} \subset f\left(A_{k-1}\right)$, and that far from the origin, all the zeros of $f$, critical points of $f$, and the Julia set of $f$ are inside the $A_{k}$ 's. To accomplish this, we will show (in a quantitative way) that $f$ is a small perturbation of the $k$ th term of the infinite product (2.1) on $A_{k}$.

Given any $s \in(1,2)$, we will choose $c$ so that $\operatorname{dim}_{\mathrm{H}}\left(\mathcal{J}\left(g^{N}\right)\right)=s$. In Section 7 , we will show that in a neighborhood of the origin, $f$ is a polynomial-like mapping which is a small perturbation of $g^{N}$. By some standard arguments using holomorphic motions, it will follow that the Julia set of the entire function $f$ will have Hausdorff dimension bounded below by a value arbitrarily close to $s$. From here, we will be able to prove that we can sort the Fatou components into two categories depending on if the component remains bounded or if the component escapes to infinity. The first type of Fatou component comes from the connected component containing the critical point 0 of $f(z)$. This component is an attracting basin which we denote by $B_{f}$. All connected components of the inverse images of $B_{f}$ are eventually mapped conformally with small distortion onto $B_{f}$ by some iterate of $f$.

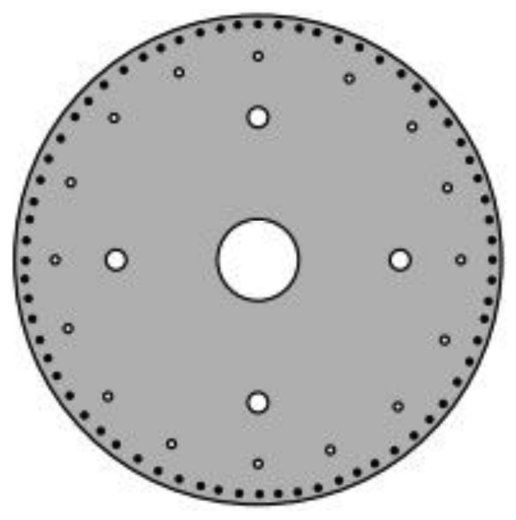

Figure 2. A round Fatou component. Such components are infinitely connected, and all the boundary components are $C^{1}$ and approximately circles. The circular boundary components accumulate onto the outermost boundary component and are arranged in layers that can be connected by approximately circular Jordan curves. This picture is not to scale; the diameters of the holes are actually much smaller than the diameter of the component.

In Sections 8 and 9 we discuss the second type of Fatou component. These are subsets of the escaping set $I(f)$, where

$$
I(f):=\left\{z: f^{n}(z) \rightarrow \infty\right\} .
$$

These components will be infinitely connected wandering domains, and their boundary components will be bounded by $C^{1}$ Jordan curves. These boundary curves will accumulate on the outermost boundary of each component. There is a distinguished central series $\left\{\Omega_{k}\right\}_{k \in \mathbb{Z}}$ of these Fatou components which surround the origin. We will split these components into two sub-categories. If $k \geq 1$, we will call $\Omega_{k}$ round since the inner and outer boundary of $\Omega_{k}$ will be $C^{1}$ Jordan curves which are approximately circles. See Figure 2. We will call $\Omega_{k}$ for $k \leq 0$ wiggly. The inner and 


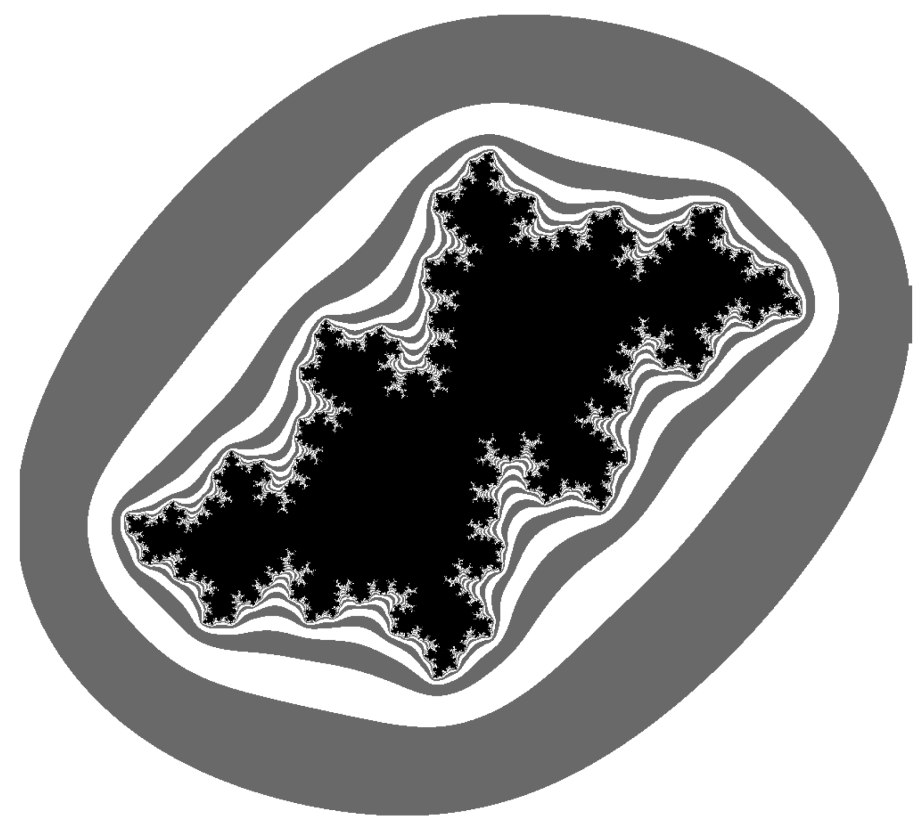

Figure 3. A schematic for a sequence of wiggly Fatou components, alternating between gray and white. The holes have been omitted to emphasize the wiggly shape of the inner and outer boundaries. These boundary components approximate level lines for the Green's function of the complement of the closure of the basin of attraction, and they surround and accumulate on the basin's fractal boundary.

outer boundary of wiggly components will be $C^{1}$ Jordan curves that approximate the fractal boundary of $B_{f}$ as $k \rightarrow-\infty$. If $\Omega_{k}$ is wiggly, then $f^{k+1}$ will map $\Omega_{k}$ to the round component $\Omega_{1}$ as a covering map. The action of $f^{k+1}$ on $\Omega_{k}$ can be thought of as first mapping $\Omega_{k}$ to a very thin annulus conformally, then to a thick annulus by a power mapping $z^{n_{1}(k+1)}$. This is similar to the dynamics on the basin of attraction containing infinity for a quadratic polynomial with connected Julia set. See Figure 3. We will see that the central series of Fatou components is the main building block for the Fatou and Julia set of $f$. Indeed, we will show all Fatou components of $f$ map conformally onto an element of the central series with small distortion.

The Julia set of $f$ will contain the boundaries of each of these two types of components. This is not the entire Julia set. Since $f$ has a multiply connected Fatou component, the work of Dominguez, Dom97, implies that the Julia set will also contain points that do not lie on the boundary of any of the Fatou components of $f$ we described above. We call these points in the Julia set buried points, and the orbits of buried points either remain bounded, belong to the bungee set, or escape slowly. In Sections 10 through 13, we will perform a detailed analysis of the Hausdorff dimension of the set of buried points. We will show that, for any small value $\epsilon_{0}$, we may define $f$ so that the Hausdorff dimension of the buried points of $f$ is at most $\epsilon_{0}$ larger than the Hausdorff dimension of the boundary of the fractal 
basin of attraction. So while the Hausdorff dimension of the buried points could possibly be strictly larger than the Hausdorff dimension of the boundary of the basin of attraction, we show that we can make this difference $\epsilon_{0}$ arbitrarily small.

To obtain an upper bound for the packing dimension, we will follow the strategy in Bis18 and study the critical exponent of a Whitney-type decomposition of the complement of the Julia set of $f$ in a bounded region. Since the Julia set of $f$ will have zero area, it turns out that this critical exponent coincides with the packing dimension, and we will show that this exponent is at most the Hausdorff dimension of the buried points. The key idea in this part of the proof is to iterate Fatou components, or pieces of Fatou components, conformally onto Fatou components where we can estimate the critical exponent directly. The trade-off is that this conformal rescaling procedure results in various corrective factors that we need to control. We do this with a combination of all the technical work done earlier in the paper.

We conclude with some notation we will use throughout the paper. We denote the complex plane by $\mathbb{C}$, we let $B(z, r)$ denote the open ball in $\mathbb{C}$ with center $z$ and radius $r$, and we set $\mathbb{D}=B(0,1)$. Likewise $C(z, r)$ will denote the circle of radius $r$ centered at $z$. We denote the closure of a set $A$ by $\bar{A}$. If $\Omega$ is a multiply connected domain in the plane, we will denote $\widehat{\Omega}$ as the union of $\Omega$ with all its bounded complementary components. In the literature, $\widehat{\Omega}$ is sometimes referred to as the polynomial hull of $\Omega$. We will say that a Jordan curve $\gamma \subset \mathbb{C}$ surrounds the origin if its bounded complementary component contains the origin. We will similarly say that a domain $\Omega$ with Jordan boundary components surrounds the origin if at least one of its boundary components surrounds the origin.

We will frequently use big-oh notation. If $\left\{x_{n}\right\}$ and $\left\{y_{n}\right\}$ are sequences in $\mathbb{C}$, then $x_{n}=O\left(y_{n}\right)$ means that there exists a constant $C$ so that $\left|x_{n}\right| \leq C\left|y_{n}\right|$ for all sufficiently large $n$. Similarly if $f(z)$ and $g(z)$ are functions, we say $f(z)=O(g(z))$ as $z \rightarrow a$ if there exists $C$ and a ball $B(a, r)$ so that $|f(z)| \leq C|g(z)|$ on $B(a, r)$. We will say that two quantities $A$ and $B$ are comparable if there exists a constant $C$ so that $C^{-1} A \leq B \leq C A$. In certain proofs, a constant $C$ may evolve throughout the proof.

\section{CONFORMAL, QUASICONFORMAL, AND POLYNOMIAL-LIKE MAPPINGS}

In this section, let $\Omega$ and $\Omega^{\prime}$ be planar domains. In this paper, we will call a mapping $f: \Omega \rightarrow \mathbb{C}$ conformal if and only if $f$ is both holomorphic and injective. If $f: \Omega \rightarrow \Omega^{\prime}$ is conformal, and $K$ is relatively compact in $\Omega$, the distortion of $f$ on $K$ is

$$
\left.D\right|_{K}:=\sup _{z, w \in K} \frac{\left|f^{\prime}(z)\right|}{\left|f^{\prime}(w)\right|} .
$$

We will often make use of the Koebe growth and distortion theorems for conformal mappings (see GM05] Theorem 4.5 p. 22) in the following form.

Lemma 3.1. Fix $r<1$, let $B=B(0, r) \subset \mathbb{D}$ be an open ball, and let $K \subset \bar{B}$ be $a$ compact set. Suppose that $f: \mathbb{D} \rightarrow \mathbb{C}$ is conformal. Then there exists a constant $C$ depending only on $r$ and not otherwise on $f$ such that

$$
C^{-1} \frac{\operatorname{diam}(K)}{\operatorname{diam}(B)} \leq \frac{\operatorname{diam}(f(K))}{\operatorname{diam}(f(B))} \leq C \frac{\operatorname{diam}(K)}{\operatorname{diam}(B)} .
$$


There exists a constant $C^{\prime}$ depending only on $r$ and not otherwise on $f$ such that

$$
\left.D\right|_{K} \leq C^{\prime}
$$

As $r \rightarrow 0$, the constants $C$ and $C^{\prime}$ tend to 1 .

In light of (3.1), when the hypotheses of Lemma 3.1 are met we will sometimes say that $f$ has bounded conformal distortion on $K$. When $r$ is close to 0 , we will sometimes say $f$ has small conformal distortion.

We call an orientation preserving homeomorphism $\varphi: \Omega \rightarrow \Omega^{\prime} K$-quasiconformal if $\varphi$ has locally square integrable distributional derivatives which satisfy

$$
\left|\varphi_{\bar{z}}(z)\right| \leq k\left|\varphi_{z}(z)\right|
$$

for almost every $z \in \Omega$, where $k=(K-1) /(K+1)<1$. We call the value $K>1$ the dilatation of $\varphi$, and when the specific value of $K$ is unimportant, we will refer to $f$ simply as quasiconformal.

We say a domain $\Omega$ is a multiply connected Jordan domain if $\Omega$ is not simply connected and all of its boundary components are Jordan curves. A domain $A \subset \mathbb{C}$ is a topological annulus if it has two complementary components, and $A$ is a Jordan annulus if its boundary components are Jordan curves. In particular, a Jordan annulus has one bounded and one unbounded complementary component. The boundary of the bounded complementary component is called the inner boundary of $A$, and the boundary of the unbounded complementary component is called the outer boundary of $A$. A round annulus is a Jordan annulus of the form $A=$ $A\left(r_{1}, r_{2}\right)=\left\{z: r_{1} \leq|z| \leq r_{2}\right\}$ where $r_{1}<r_{2}$. Given a Jordan annulus $A$, there exists a unique $r \in(1, \infty)$ and a conformal mapping

$$
\varphi: A \rightarrow \operatorname{int} A(1, r),
$$

where int $A(1, r)$ is defined to be the interior of $A(1, r)$. We define the modulus of $A$ to be $\bmod (A)=\frac{1}{2 \pi} \log (r)$. The modulus of an annulus is a quasi-invariant: if $\varphi: A \rightarrow A^{\prime}$ is a quasiconformal homeomorphism between two Jordan annuli, then

$$
\frac{1}{K} \bmod (A) \leq \bmod \left(A^{\prime}\right) \leq K \bmod (A) .
$$

Finally, we remark that using our definition of conformality, $f$ is conformal if and only if $f$ is 1-quasiconformal. In particular the modulus of an annulus is invariant under conformal mappings. This allows for the following invariant formulation of Lemma 3.1 .

Lemma 3.2. Let $\Omega$ be simply connected, let $U$ be open and compactly contained in $\Omega$, and let $K$ be a compact subset of $\bar{U}$. Suppose $f: \Omega \rightarrow \Omega^{\prime}$ is conformal. Then there is a constant $C$ which depends only on $\bmod (\Omega \backslash \bar{U})$ so that

$$
C^{-1} \frac{\operatorname{diam}(K)}{\operatorname{diam}(U)} \leq \frac{\operatorname{diam}(f(K))}{\operatorname{diam}(f(U))} \leq C \frac{\operatorname{diam}(K)}{\operatorname{diam}(U)} .
$$

There exists a constant $C^{\prime}$ which depends only on $\bmod (\Omega \backslash \bar{U})$ so that

$$
\left.D\right|_{\bar{U}} \leq C^{\prime}
$$

As $\bmod (\Omega \backslash \bar{U}) \rightarrow 0$, the constants $C$ and $C^{\prime}$ tend to 1 . 
A Jordan curve $\Gamma \subset \mathbb{C}$ is called a $\kappa$-quasicircle if $\kappa>1$, and for all points $z, w \in \Gamma$, if $\gamma$ denotes the subarc of $\Gamma$ of smallest diameter with endpoints $z$ and $w$, we have

$$
\operatorname{diam}(\gamma) \leq \kappa|z-w|
$$

$\Gamma$ is a quasicircle if and only if there exists a quasiconformal mapping $\varphi: \mathbb{C} \rightarrow \mathbb{C}$ that maps the unit circle onto $\Gamma$.

Douady and Hubbard introduced polynomial-like mappings in DH85. Recall that a continuous mapping $f: \Omega \rightarrow \Omega^{\prime}$ is called proper if the inverse image of every compact set $K \subset \Omega^{\prime}$ is compact in $\Omega$. A degree $d$ polynomial-like map is a triple $\left(f, \Omega, \Omega^{\prime}\right)$, where $f: \Omega \rightarrow \Omega^{\prime}$ is a proper holomorphic mapping of degree $d$, and $\Omega$ and $\Omega^{\prime}$ are bounded Jordan domains with $\Omega$ relatively compact in $\Omega^{\prime}$. We define the filled Julia set of $f$ by

$$
K_{f}:=\bigcap_{n \geq 0} f^{-n}(\Omega) .
$$

The filled Julia set is precisely the set of points that remain in $\Omega$ for all iterates of $f$. The Julia set of $f$ is defined to be the boundary $\partial K_{f}$, and we denote it by $J_{f}$. The straightening lemma of Douady and Hubbard is of great importance, and we will need the following simple formulation.

Theorem 3.3 (The straightening lemma). Let $\left(f, \Omega, \Omega^{\prime}\right)$ be a degree d polynomiallike mapping. Then there exists a quasiconformal mapping $\varphi: \mathbb{C} \rightarrow \mathbb{C}$ and a polynomial $p$ of degree $d$ so that for all $z \in \Omega$ we have

$$
f(z)=\varphi \circ p \circ \varphi^{-1}(z) .
$$

A polynomial-like mapping $\left(f, \Omega, \Omega^{\prime}\right)$ is called hyperbolic if every critical point of $f$ contained in $K_{f}$ is attracted to an attracting cycle. Equivalently, $\left(f, \Omega, \Omega^{\prime}\right)$ is hyperbolic if there exists $m \in \mathbb{N}$ so that $\left|\left(f^{m}\right)^{\prime}\right|>1$ on $J_{f}$. In this paper, the polynomial-like mappings will arise as the restriction of entire functions, and it will be important that we distinguish between hyperbolicity of polynomial-like mappings, versus hyperbolicity as a transcendental entire function (which our example cannot be, since we will see that it has an unbounded set of critical values. See [RGS17.)

\section{Dimension And Whitney-type Decompositions}

Given a set $A \subset \mathbb{C}$, we define its $\alpha$-Hausdorff measure to be the quantity

$H^{\alpha}(A):=\lim _{\delta \rightarrow 0} H_{\delta}^{\alpha}(A):=\lim _{\delta \rightarrow 0}\left(\inf \left\{\sum_{i=1}^{\infty} \operatorname{diam}\left(U_{i}\right)^{\alpha}: A \subset \bigcup_{i=1}^{\infty} U_{i}, \operatorname{diam}\left(U_{i}\right)<\delta\right\}\right)$.

The infimum is taken over all countable covers of $A$ by sets $\left\{U_{i}\right\}_{i=1}^{\infty}$. One can check that if $H^{t}(A)<\infty$, then $H^{s}(A)=0$ for all $s>t$, and similarly, if $H^{t}(A)>0$, then $H^{s}(A)=\infty$ for all $s<t$. It follows that the Hausdorff dimension

$$
\operatorname{dim}_{\mathrm{H}}(A):=\sup \left\{t: H^{t}(A)=\infty\right\}=\inf \left\{t: H^{t}(A)=0\right\}
$$

is uniquely defined.

Given a compact set $K \subset \mathbb{C}$, define $N(K, \epsilon)$ to be the minimal number of open balls of radius $\epsilon$ needed to cover $K$. Since $K$ is compact, this number exists and is finite. We define the upper Minkowski dimension of $K$ to be

$$
\overline{\operatorname{dim}_{\mathrm{M}}}(K)=\limsup _{\epsilon \rightarrow 0} \frac{\log (N(K, \epsilon))}{\log (1 / \epsilon)}=\sup \left\{s \geq 0: \limsup _{\epsilon \rightarrow 0} N(K, \epsilon) \epsilon^{s}=0\right\} .
$$


One obtains an equivalent definition using squares of side length $\epsilon$ to define $N(K, \epsilon)$. For this reason, upper Minkowski dimension is often referred to as upper box counting dimension in the literature.

We define the packing dimension of $K$ to be

$$
\operatorname{dim}_{\mathrm{P}}(K)=\inf \left\{\sup _{i \in \mathbb{N}}\left\{\overline{\operatorname{dim}_{\mathrm{M}}} K_{i}: K=\bigcup_{i=1}^{\infty} K_{i}\right\}\right\} .
$$

Here, the infimum is taken over all coverings of $K$ by countably many compact subsets $K_{i}$. Note that we do not require $K$ itself to be compact.

In this paper, we will investigate the upper Minkowski and packing dimension of unbounded Julia sets, so strictly speaking, Definition (4.2) does not make sense. We can instead consider the local upper Minkowski dimension of the Julia set, which is the upper Minkowski dimension of the Julia set intersected with an open neighborhood of finite diameter. In RS05, Rippon and Stallard show that the local upper Minkowski dimension of the Julia set of an entire function is constant and coincides with its packing dimension, except perhaps in a neighborhood of 1 point (a point with finite backward orbit; there is at most 1 by the Picard theorem). Our example will not have an exceptional value of this kind, so their result further implies that the packing dimension and local upper Minkowski dimension are the same, no matter where we compute the local upper Minkowski dimension. In light of this, we will abuse notation and refer to the local upper Minkowski dimension of $\mathcal{J}(f)$ by $\overline{\operatorname{dim}_{M}}(\mathcal{J}(f))$; the neighborhood we are using will always be made clear.

A detailed discussion of these dimensions can be found in Bis18. The survey Sta08. also contains a detailed discussion of the above definitions, along with an overview of many results about the dimension of Julia sets of transcendental entire functions. We focus instead on a detailed discussion of Whitney-type decompositions, which will be our primary tool in estimating the packing dimension.

An interval $I \subset \mathbb{R}$ is called dyadic if $I=\left[j / 2^{n},(j+1) / 2^{n}\right]$ for integers $j$ and $n$. We denote the set of all dyadic intervals by $\Delta$, and all the dyadic intervals of side length $2^{-n}$ by $\Delta_{n}$. A dyadic square $Q$ in the plane is the product of two dyadic intervals in $\Delta_{n}$.

Definition 4.1. Let $F \subset \mathbb{C}$ be a nonempty closed set, and let $\Omega=\mathbb{C} \backslash F$. A Whitney decomposition of $\Omega$ is a countable collection of dyadic squares $\left\{Q_{j}\right\}_{j=1}^{\infty}$ satisfying the following three properties:

(1) $\Omega=\bigcup_{j=1}^{\infty} \overline{Q_{j}}$.

(2) For all $j \neq k, Q_{j}$ and $Q_{k}$ have disjoint interior.

(3) There exists a constant $C>1$ so that for all $j \geq 1$,

$$
\frac{1}{C} \operatorname{dist}\left(Q_{j}, \partial \Omega\right) \leq \operatorname{diam}\left(Q_{j}\right) \leq C \operatorname{dist}\left(Q_{j}, \partial \Omega\right) .
$$

Whitney decompositions always exist when $F$ is nonempty, (see [Ste70], p. 167). We may always choose the constant $C=4$. For our purposes, the key feature of Whitney decompositions will be that the dyadic squares are approximately squares with unit area with respect to the hyperbolic metric on the connected components of $\Omega$. It is often advantageous to consider more abstract decompositions with similar properties where the elements will not necessarily be dyadic squares.

Definition 4.2. Let $F \subset \mathbb{C}$ be a nonempty closed set, $\Omega=\mathbb{C} \backslash F$, and let $C>1$ and $\lambda \geq 1$. A $(C, \lambda)$-Whitney-type decomposition of $\Omega=\mathbb{C} \backslash F$ is a countable collection 
of open sets $\left\{S_{j}\right\}_{j=1}^{\infty}$ whose boundaries are quasicircles that satisfy the following four properties:

(1) $\Omega=\bigcup_{j=1}^{\infty} \overline{S_{j}}$.

(2) For all $j \neq k, S_{j}$ and $S_{k}$ are disjoint.

(3) There exists a constant $C>1$ so that for all $j \geq 1$,

$$
\frac{1}{C} \operatorname{dist}\left(S_{j}, \partial \Omega\right) \leq \operatorname{diam}\left(S_{j}\right) \leq C \operatorname{dist}\left(S_{j}, \partial \Omega\right) .
$$

(4) There exists a constant $\lambda$ so that for all $j \geq 1$ we have

$$
\frac{\operatorname{diam}\left(S_{j}\right)^{2}}{\operatorname{Area}\left(S_{j}\right)} \leq \lambda \text {. }
$$

For convenience we will often omit the constants and refer to such collections as Whitney-type decompositions, and we will still refer to the elements of a Whitneytype decomposition as squares. Note that the Whitney decomposition of dyadic squares described above is a $(4,2)$-Whitney-type decomposition. Whenever we summon a Whitney-type decomposition in a proof, unless stated otherwise, we will assume it is the $(4,2)$-Whitney-type decomposition of dyadic squares. A Whitneytype decomposition of a given open set $\Omega$ is defined by taking $F=\mathbb{C} \backslash \Omega$.

Definition 4.3. Let $F \subset \mathbb{C}$ be a nonempty compact set and $\Omega=\mathbb{C} \backslash F$. A Whitney-type decomposition of a neighborhood of $F$ is a countable collection of open sets $\left\{S_{j}\right\}_{j=1}^{\infty}$ whose boundaries are quasicircles that satisfy the following four properties:

(1) The sets $\left\{S_{j}\right\}_{j=1}^{\infty}$ cover a neighborhood of $F$; there exists $d>0$ so that

$$
\{z: \operatorname{dist}(z, F)<d\} \subset \bigcup_{j=1}^{\infty} \overline{S_{j}} .
$$

(2) For all $j \neq k, S_{j}$ and $S_{k}$ are disjoint.

(3) There exists a constant $C>1$ so that for all $j \geq 1$,

$$
\frac{1}{C} \operatorname{dist}\left(S_{j}, \partial \Omega\right) \leq \operatorname{diam}\left(S_{j}\right) \leq C \operatorname{dist}\left(S_{j}, \partial \Omega\right) .
$$

(4) There exists a constant $\lambda$ so that for all $j \geq 1$ we have

$$
\frac{\operatorname{diam}\left(S_{j}\right)^{2}}{\operatorname{Area}\left(S_{j}\right)} \leq \lambda \text {. }
$$

Example 4.4. It is often useful to create Whitney-type decompositions welladapted to the dynamics of polynomial maps. Let $f(z)=z^{N}$, where $N \in \mathbb{N}$ and $N \geq 2$. We will create a Whitney-type decomposition of a neighborhood of $\overline{B(0,1)}$ using the dynamics of $f$.

Let $n \geq 0$ and $R>1$, and let $C_{n}$ denote the circle $C\left(0, R^{1 / N^{n}}\right)$. Then $f\left(C_{n}\right)=$ $C_{n-1}$ and $f^{n}\left(C_{n}\right)=C_{0}$ for all $n \geq 1$. For $n \geq 1$, let $A_{n}$ denote the open round annulus with inner boundary $C_{n}$ and outer boundary $C_{n-1}$. Then $A_{n}$ decomposes into $N^{n}$ many sets $S_{n, j}$, where

$$
f^{n}: S_{n, j} \rightarrow \operatorname{int} A\left(R^{N}, R\right) \backslash \mathbb{R}^{\geq 0}
$$

is conformal. See Figure 4 . 
There exists constants $C$ and $\lambda$ that depend only on $N$ and $R$ such that the collection $\left\{S_{j, n}\right\}$ is a $(C, \lambda)$-Whitney decomposition. The Whitney-type decomposition is dynamical in the sense that if $S_{j, n} \subset A_{n}$ is a component above, $f\left(S_{j, n}\right)=$ $S_{j^{\prime}, n-1} \subset A_{n-1}$ for some $j^{\prime}$.

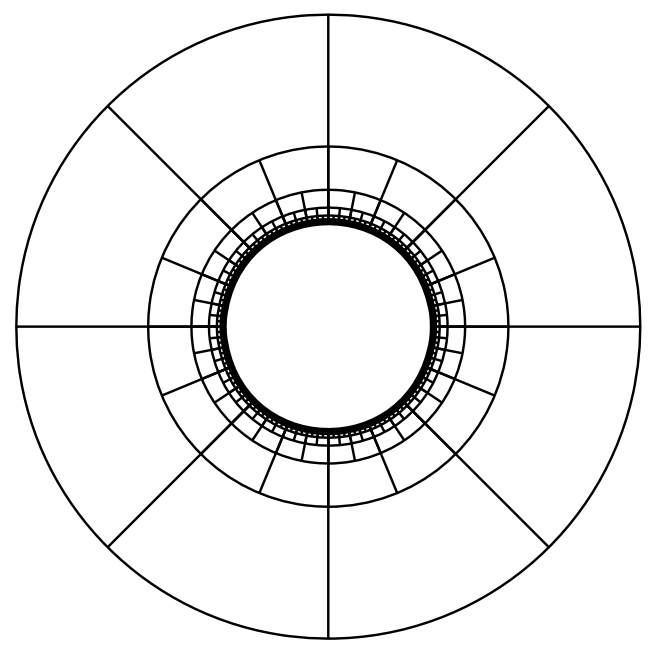

Figure 4. The Whitney-type decomposition in Example 4.4 for the case $N=2$. In this picture the "squares" form a Whitney-type decomposition for a neighborhood of $\overline{B(0,1)}$. Each square in the picture has the property $z^{2}$ maps a square in one ring of squares to one in the next ring, normalized so that one of the squares in each ring has a radial segment on the real axis.

The following is a simple but very useful geometric lemma.

Lemma 4.5. Let $W_{1}(\Omega)$ and $W_{2}(\Omega)$ be $\left(C_{j}, \lambda_{j}\right)$-Whitney-type decompositions of $\Omega$ for $j=1,2$. Then there exists a constant $L=L\left(C_{1}, C_{2}, \lambda_{1}, \lambda_{2}\right)$ such that if $Q \in W_{1}(\Omega)$, then $Q$ is covered by at most $L$ elements $S \in W_{2}(\Omega)$, and vice versa.

The key to the proof of Lemma 4.5 is comparing the area of a square $Q$ in one collection with the area of all the squares in the other collection that intersect $Q$. We omit the details.

Let $K \subset \mathbb{C}$ be compact, and let $\Omega=\mathbb{C} \backslash K$. Let $W(\Omega)$ be a Whitney-type decomposition of $\Omega$. We define the critical exponent of $K$ to be

$$
\alpha(K)=\inf \left\{\alpha \geq 0: \sum_{Q \in W(\Omega)} \operatorname{diam}(Q)^{\alpha}<\infty, \operatorname{diam}(Q)<1\right\} .
$$

The critical exponent does not depend on the Whitney-type decomposition.

Lemma 4.6. Suppose that $W(\Omega)$ and $W^{\prime}(\Omega)$ are Whitney-type decompositions of a domain $\Omega$. Then

$$
\sum_{Q \in W(\Omega)} \operatorname{diam}(Q)^{\alpha}<\infty \quad \text { if and only if } \sum_{Q \in W^{\prime}(\Omega)} \operatorname{diam}(Q)^{\alpha}<\infty .
$$

Proof. This follows immediately from Lemma 4.5. 
Given a Whitney-type decomposition, we will sometimes call the sum of diameters of the squares as in Lemma 4.6 an $\alpha$-Whitney sum. Thus $\alpha(K) \leq \alpha$ if and only if the $t$-Whitney sums for some Whitney-type decomposition of the complement of $K$ converge for all $t>\alpha$.

The critical exponent will is the main tool we will use to estimate the packing dimension. The following is Lemma 2.6.1 in BP17.

Lemma 4.7. Let $K \subset \mathbb{C}$ be compact. Then $\alpha(K) \leq \overline{\operatorname{dim}_{\mathrm{M}}}(K)$, and if $K$ has zero area, then $\alpha(K)=\overline{\operatorname{dim}_{\mathrm{M}}}(K)$.

In this paper, the fundamental Whitney-type decompositions we consider are decompositions with dyadic squares, and the decomposition in Example 4.4 It will also be important that these Whitney-type decompositions behave well under conformal and quasiconformal mappings.

Lemma 4.8. Let $f: \Omega \rightarrow \Omega^{\prime}$ be a K-quasiconformal homeomorphism of two domains $\Omega$ and $\Omega^{\prime}$. Let $W(\Omega)$ be a $(C, \lambda)$-Whitney-type decomposition for $\Omega$. Define

$$
f(W(\Omega)):=\left\{f\left(Q_{j}\right): Q_{j} \in W(\Omega)\right\} .
$$

Then $f(W(\Omega))$ is a $\left(C^{\prime}, \lambda^{\prime}\right)$-Whitney-type decomposition of $\Omega^{\prime}$, and the constants $C$ and $\lambda$ only depend on the constant $K$ and on the constants $C$ and $\lambda$.

It follows that conformal and quasiconformal mappings take Whitney-type decompositions to new Whitney-type decompositions. When $f$ is conformal, then Lemma 4.8 follows from the Lemma 3.2. When $f$ is $K$-quasiconformal, Lemma 4.8 follows from Theorem 11.14 in Hei01. One can also argue directly by applying standard modulus of path family arguments to the topological annuli $\Omega \backslash \bar{Q}_{j}$ for each square $Q_{j} \in W(\Omega)$.

Theorem 4.9. Let $f: \Omega \rightarrow \Omega^{\prime}$ be a K-quasiconformal homeomorphism. Let $W(\Omega)$ and $W\left(\Omega^{\prime}\right)$ be Whitney-type decompositions of $\Omega$ and $\Omega^{\prime}$, respectively. Then there exists a constant $L$ that depends only on $K$ and the constants defining the Whitneytype decompositions so that each $S \in f(W(\Omega))$ is covered by at most $L$ elements $Q \in W\left(\Omega^{\prime}\right)$.

Proof. This follows immediately by combining Lemma 4.5 and Lemma 4.8

We will use Corollary 4.10 often.

Corollary 4.10. Let $f: \Omega \rightarrow \Omega^{\prime}$ be $K$-quasiconformal. Then the $\alpha$-Whitney sums of $W\left(\Omega^{\prime}\right)$ and $f(W(\Omega))$ are comparable with constant depending only on $K$ and on the constants defining the Whitney-type decompositions.

\section{The Definition of $f$}

In this section, we specify the parameters defining $f$ and show that it is an entire function. Recall that the main cardioid of the Mandelbrot set is the region consisting of all parameters $c=\frac{\mu}{2}\left(1-\frac{\mu}{2}\right)$, where $\mu \in \mathbb{D}$. If $c$ is a parameter in the main cardioid, the Julia set of $z^{2}+c$ is a quasicircle with an attracting fixed point in its interior. For each $s \in(1,2)$, we may choose $c$ in the main cardioid so that $\operatorname{dim}_{\mathrm{H}}\left(\mathcal{J}\left(z^{2}+c\right)\right)=\operatorname{dim}_{\mathrm{P}}\left(\mathcal{J}\left(z^{2}+c\right)\right)=\overline{\operatorname{dim}_{\mathrm{M}}}\left(\mathcal{J}\left(z^{2}+c\right)\right)=s$ (see Shi98] p.232 and Sul83 p.742, along with Theorem 7.6.7 in PU10). 
Having chosen such a $c$, recall that we defined $g(z)=z^{2}+c$, and $g^{N}(z)$ denotes the $N$ th iterate of $g$. Since $g^{N}$ is a degree $2^{N}$ monic polynomial there exists some $R>0$ so that if $|z| \geq R$ we have

$$
\frac{1}{2} \leq\left|\frac{g^{N}(z)}{z^{2^{N}}}\right| \leq 2
$$

We will always assume $R$ is big enough so that (5.1) holds. by

Next, given some integer $N>0$, define a sequence of integers for $k=0,1,2 \ldots$

$$
n_{k}:=2^{N+k-1} .
$$

Note that when $k \neq 0$ we have $n_{k} \geq 2^{N}$, we have $n_{1}=2^{N}$, and for all $k$ we have $2 n_{k}=n_{k+1}$. Given the value $R$ above, define

$$
R_{1}=2 R
$$

We will construct our infinite product as a sequence of partial products inductively as follows. Given $R$ as above we can define

$$
\begin{aligned}
F_{1}(z):=\left(1-\frac{1}{2}\left(\frac{z}{R_{1}}\right)^{n_{1}}\right), \\
f_{1}(z):=g^{N}(z) \cdot F_{1}(z), \\
R_{2}:=M\left(f_{1}, 2 R_{1}\right):=\max \left\{\left|f_{1}(z)\right|:|z|=2 R_{1}\right\} .
\end{aligned}
$$

Next, assume that $f_{k-1}, F_{k-1}$ and $R_{k}$ have all been defined for some $k \geq 2$. From there, we may define

$$
\begin{gathered}
F_{k}(z):=\left(1-\frac{1}{2}\left(\frac{z}{R_{k}}\right)^{n_{k}}\right), \\
f_{k}(z):=g^{N}(z) \prod_{j=1}^{k} F_{j}(z)=f_{k-1}(z) \cdot F_{k}(z), \\
R_{k+1}:=M\left(f_{k}, 2 R_{k}\right)=\max \left\{\left|f_{k}(z)\right|:|z|=2 R_{k}\right\} .
\end{gathered}
$$

To prove that $f$ defines an entire function, we first must record some basic facts about the growth rate of $\left\{R_{k}\right\}_{k=1}^{\infty}$ and $\left\{n_{k}\right\}_{k=1}^{\infty}$. Lemma 5.1 is completely elementary but used often. We only remark that (3) below is just a restatement of (2).

Lemma 5.1 (The growth rate of $n_{k}$ ). For all $k=1,2, \ldots$, we have

(1) $n_{k}=2 n_{k-1}$, and $n_{k} \geq 2^{N}$.

(2) $2^{N}+\sum_{j=1}^{k} n_{j}=n_{k+1}$.

(3) $\operatorname{deg}\left(f_{k}\right)=2 \operatorname{deg}\left(F_{k}\right)$.

Lemma 5.2 (The growth rate of $R_{k}$ ). Suppose that $R$ is so large that (5.1) holds. Then if $N \geq 10$, for all $k \geq 1$ we have

$$
R_{k+1} \geq 2^{n_{k}} R_{k}^{2^{N-1}+n_{k-1}} \geq 2^{N} R_{k}^{2^{N}} .
$$


Proof. By (5.1), when $N \geq 10$ we have,

$$
\begin{aligned}
R_{2} & =\max _{|z|=2 R_{1}}\left|f_{1}(z)\right|=\max _{|z|=2 R_{1}}\left|g^{N}(z)\right| \cdot\left|\left(1-\frac{1}{2} \frac{z^{n_{1}}}{R_{1}^{n_{1}}}\right)\right| \\
& \geq \frac{1}{2} \cdot\left(2 R_{1}\right)^{2^{N}} \cdot \max _{|z|=2 R_{1}}\left|\left(1-\frac{1}{2} \frac{z^{n_{1}}}{R_{1}^{n_{1}}}\right)\right| \\
& \geq \frac{1}{2} \cdot 2^{n_{1}} \cdot R_{1}^{n_{1}} \cdot\left(2^{n_{1}-1}-1\right) \\
& \geq 2^{2 n_{1}-3} R_{1}^{2^{N}} \geq 2^{n_{1}} R_{1}^{2^{N-1}+n_{0}} .
\end{aligned}
$$

This is the base case for an induction. Suppose that for some $k \geq 3$, and for all $2 \leq j \leq k$, we have

$$
R_{j} \geq 2^{n_{j-1}} R_{j-1}^{2^{N-1}+n_{j-2}} \geq 2^{N} R_{j-1}^{2^{N}} \geq 4 R_{j-1}^{2} .
$$

This induction hypothesis implies that $R_{k}^{1 / 2} \geq R_{j}$ for all $j \leq k-1$. Therefore,

$$
\begin{aligned}
R_{k+1} & \geq \max _{|z|=2 R_{k}}\left|f_{k}(z)\right|=\max _{|z|=2 R_{k}}\left|g^{N}(z)\right| \cdot \prod_{j=1}^{k}\left|\left(1-\frac{1}{2} \frac{z^{n_{j}}}{R_{j}^{n_{j}}}\right)\right| \\
& \geq \frac{1}{2} \cdot 2^{2^{N}} \cdot R_{k}^{2^{N}} \cdot \prod_{j=1}^{k}\left(2^{n_{j}-1} \frac{R_{k}^{n_{j}}}{R_{j}^{n_{j}}}-1\right) \\
& \geq \cdot 2^{2^{N}-1} \cdot R_{k}^{2^{N}} \cdot \prod_{j=1}^{k}\left(2^{n_{j}-2} \frac{R_{k}^{n_{j}}}{R_{j}^{n_{j}}}\right) \\
& \geq 2^{2^{N}-1} \cdot R_{k}^{2^{N}} \cdot 2^{n_{k}-2} \prod_{j=1}^{k-1}\left(2^{n_{j}-2} R_{k}^{n_{j-1}}\right) \\
& \geq 2^{2^{N}-2 k-1+\sum_{j=1}^{k} n_{j}} \cdot R_{k}^{2^{N}+\sum_{j=1}^{k-1} n_{j-1}} .
\end{aligned}
$$

To conclude, we use Lemma 5.1 and the assumption that $N \geq 10$ to see that

$$
\begin{aligned}
R_{k+1} & \geq 2^{2^{N}-2 k+n_{k+1}} \cdot R_{k}^{2^{N-1}+n_{k-1}} \\
& \geq 2^{2^{N}+n_{k}} \cdot R_{k}^{2^{N-1}+n_{k-1}} .
\end{aligned}
$$

This completes the proof.

For the rest of the paper, we will always assume that $N \geq 10$, so that the conclusion of Lemma 5.2 is always valid. Lemma 5.2 also implies the following simpler inequalities that will often be sufficient for our purposes.

Corollary 5.3 (Other useful inequalities). For $k \geq 1$ we have

(1) $R_{k+1} \geq 4 R_{k}^{2}$.

(2) $R_{k+1} \geq(2 R)^{2^{k N}}$.

The proof of (1) is obvious, and the proof of (2) is a simple induction; see Corollary 8.3 of [Bis18]. Corollary 5.3 allows us to apply the same argument as Lemma 5.2 in Bis18 and conclude that $f(z)$ is a transcendental entire function. 
Corollary 5.4. The function

$$
f(z)=g^{N}(z) \cdot \prod_{k=1}^{\infty} F_{k}(z)
$$

converges uniformly on compact subsets of $\mathbb{C}$. In particular, $f(z)$ is a transcendental entire function.

We conclude this section by recording some useful estimates regarding the relative growth rates of $\left\{R_{k}\right\}_{k=1}^{\infty}$. The proof follows from Lemma 5.2 and a use of Taylor series approximations, and we refer the reader to Sections 6 and 8 of [Bis18] for the details.

Lemma 5.5. Suppose that $\left\{R_{k}\right\}_{k=1}^{\infty}$ has been defined as in this section, and $m \geq 1$. Then

$$
\begin{gathered}
\prod_{j=1}^{k-1}\left(1+\left(\frac{R_{j}}{R_{k}}\right)^{m}\right)=1+O\left(R_{k}^{-m / 2}\right), \\
\prod_{j=k+1}^{\infty}\left(1+\frac{R_{k}}{R_{j}}\right)=1+O\left(R_{k}^{-1}\right) .
\end{gathered}
$$

Finally, if $|z| \leq 4 R_{k}$, we have

$$
\prod_{j=k+1}^{\infty} F_{j}(z)=1+O\left(R_{k}^{-1}\right) .
$$

\section{THE MAPPING BEHAVIOR OF $f$ AWAY FROM THE ORIGIN}

We now move on to analyzing the function $f$ far away from the origin. The purpose of this section is to show that $f$ behaves like simpler functions on suitably defined regions of $\mathbb{C}$. To be more specific, recall that

$$
f(z):=g^{N}(z) \cdot \prod_{j=1}^{\infty} F_{j}(z) .
$$

We will show that we can decompose $\mathbb{C} \backslash B\left(0, R_{1} / 4\right)$ into regions where $f$ looks approximately like the $j$ th term of the infinite product multiplied by a monomial. The observations and estimates here are vital for understanding to precise dynamical behavior of $f$.

We define

$$
H_{m}(z)=z^{m}\left(2-z^{m}\right) .
$$

A detailed description of the conformal mapping behavior of $H_{m}$ can be found in Section 9 of Bis18. For our purposes, we will need to consider the connected components of $\mathbb{C} \backslash\left\{\left|H_{m}(z)\right|=1\right\}$. This set has $m+2$ connected components, one unbounded, one containing the origin, and $m$ simply connected regions which we call petals. We denote a single petal by $\Omega_{m}^{p}$. Then $H_{m}: \Omega_{m}^{p} \rightarrow \mathbb{D}$ is a conformal mapping, and $\operatorname{diam}\left(\Omega_{m}^{p}\right)=O(1 / m)$. See Figure 5 

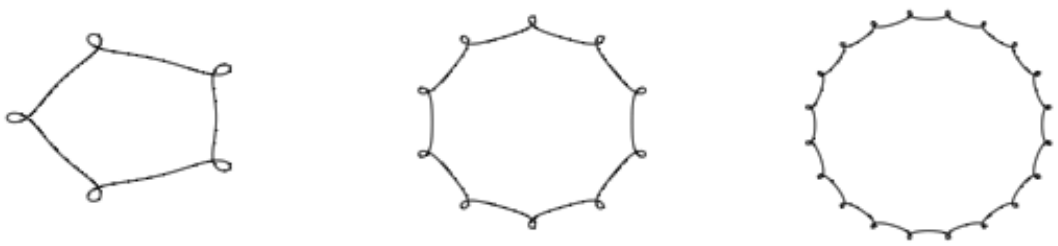

Figure 5. An illustration of the level sets of $\left\{\left|H_{m}(z)\right|=1\right\}$ for $m=5,10$ and 20 . There are $m$ petals where $H_{m}$ is a conformal mapping to the disk, and as $m$ grows, the diameter of the petals shrinks. All the points on $\left\{\left|H_{m}(z)\right|=1\right\}$ are distance $O(1 / m)$ from the unit circle $|z|=1$.

Next, we decompose $\mathbb{C} \backslash B\left(0, R_{1} / 4\right)$ into annuli as follows. For $k \geq 1$, define

$$
\begin{aligned}
A_{k}:=\left\{z: \frac{1}{4} R_{k} \leq|z| \leq 4 R_{k}\right\}, & B_{k}:=\left\{z: 4 R_{k} \leq|z| \leq \frac{1}{4} R_{k+1}\right\}, \\
V_{k}:=\left\{z: \frac{3}{2} R_{k} \leq|z| \leq \frac{5}{2} R_{k}\right\}, & U_{k}:=\left\{z: \frac{5}{4} R_{k} \leq|z| \leq 3 R_{k}\right\} .
\end{aligned}
$$

Note that $V_{k}$ is compactly contained inside of $U_{k}$. See Figure 6 .

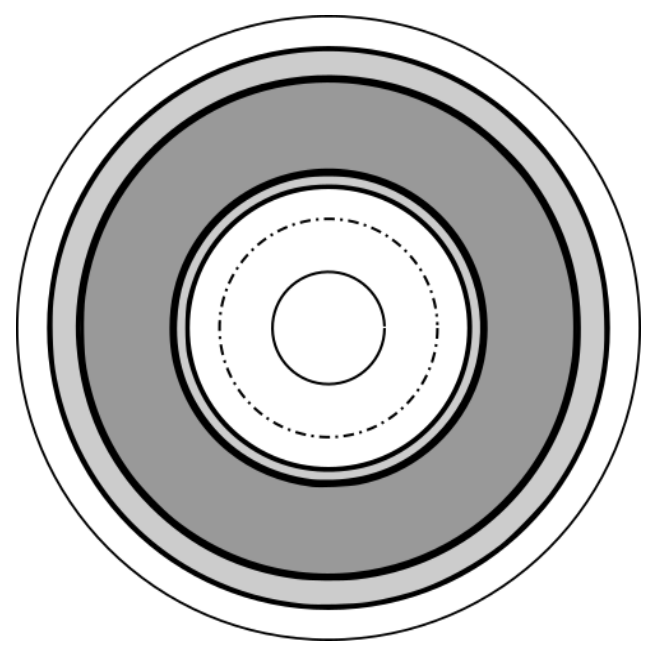

Figure 6. A schematic for $A_{k}, k \geq 1$. The innermost circle and outermost circle form the boundary of $A_{k}$. The dashed line is the circle $|z|=R_{k}$. The lightly shaded region is $U_{k}$, and the darker region is $V_{k}$, which is compactly contained in $U_{k}$. In the upcoming sections, we will see that the Julia set of $f$ is contained near the circle $|z|=R_{k}$ and in $V_{k}$.

The following is Lemma 10.1 in Bis18. We include its simple proof. 
Lemma 6.1. With $H_{m}$ defined above, for all integers $k \geq 1$ we have

$$
F_{k}(z)=\frac{1}{2}\left(\frac{R_{k}}{z}\right)^{n_{k}} H_{n_{k}}\left(\frac{z}{R_{k}}\right) .
$$

Proof. We compute this directly by factoring as follows,

$$
\begin{aligned}
\frac{1}{2}\left(\frac{R_{k}}{z}\right)^{n_{k}} H_{n_{k}}\left(\frac{z}{R_{k}}\right) & =\frac{1}{2}\left(\frac{R_{k}}{z}\right)^{n_{k}}\left(\frac{z}{R_{k}}\right)^{n_{k}}\left(2-\left(\frac{z}{R_{k}}\right)^{n_{k}}\right) \\
& =\left(\frac{R_{k}}{z}\right)^{n_{k}}\left(\frac{z}{R_{k}}\right)^{n_{k}}\left(1-\frac{1}{2}\left(\frac{z}{R_{k}}\right)^{n_{k}}\right) \\
& =F_{k}(z) .
\end{aligned}
$$

This is exactly what we wanted.

Lemma 6.2 says that $f$ looks like a slightly perturbed multiple of $H_{n_{k}}$ on the annuli $A_{k}$.

Lemma 6.2. Let $k \geq 1$. If $z \in A_{k}$, there is a constant $C_{k}$ so that

$$
f(z)=C_{k} H_{n_{k}}\left(\frac{z}{R_{k}}\right)\left(1+O\left(R_{k}^{-1}\right)\right) .
$$

For $k \geq 2$, the constant $C_{k}$ is given by the formula

$$
C_{k}=(-1)^{k-1} 2^{-k} R_{k}^{n_{k}} \prod_{j=1}^{k-1} R_{j}^{-n_{j}} .
$$

For $k=1$ the constant is given by

$$
C_{1}=\frac{1}{2} R_{1}^{n_{1}}
$$

The proof is almost exactly the same as Lemma 10.2 in [Bis18. The idea is very simple. We break $f$ into the product of three pieces:

$$
f(z)=\left(g^{N}(z) \cdot \prod_{j=1}^{k-1} F_{j}(z)\right) \cdot F_{k}(z) \cdot\left(\prod_{j=k+1}^{\infty} F_{j}(z)\right) .
$$

The first term of (6.1) is $g^{N}$ followed by the the first $k-1$ terms of the infinite product. That is estimated by some factoring and applying (5.3) from Lemma 5.5. The second piece of (6.1) is just $F_{k}$, which we rewrite using Lemma 6.1. The third piece of (6.1) is the tail of the infinite product, which we estimate using (5.5) from Lemma 5.5.

Lemma 6.3 says that $f$ looks like a power function on $B_{k}$. This fact is used but not proved directly in [Bis18, so we include it for completeness.

Lemma 6.3. Let $k \geq 1$. Then for all $z \in B_{k}$, we have

$$
f(z)=-C_{k}\left(\frac{z}{R_{k}}\right)^{2 n_{k}}\left(1+O\left(R_{k+1}^{-1}\right)\right) \cdot\left(1+O\left(4^{-n_{k+1}}\right)\right) \cdot\left(1+O\left(4^{-n_{k}}\right)\right) .
$$

Proof. The reasoning above and a similar decomposition to (6.1) allow us to conclude that for all $z \in B_{k}$,

$$
f(z)=C_{k} H_{n_{k}}\left(\frac{z}{R_{k}}\right) F_{k+1}(z)\left(1+O\left(R_{k+1}^{-1}\right)\right) .
$$


The only change is that we keep the $F_{k+1}(z)$ term. However, when $z \in B_{k}$, we have $4 R_{k} \leq|z| \leq R_{k+1} / 4$, so that,

$$
\begin{aligned}
H_{n_{k}}\left(\frac{z}{R_{k}}\right) & =\left(\frac{z}{R_{k}}\right)^{n_{k}}\left(2-\left(\frac{z}{R_{k}}\right)^{n_{k}}\right) \\
& =\left(\frac{z}{R_{k}}\right)^{2 n_{k}}\left(2\left(\frac{R_{k}}{z}\right)^{n_{k}}-1\right) \\
& =-\left(\frac{z}{R_{k}}\right)^{2 n_{k}}\left(1+O\left(4^{-n_{k}}\right)\right) .
\end{aligned}
$$

A similar computation yields

$$
F_{k+1}(z)=\left(1-\frac{1}{2}\left(\frac{z}{R_{k+1}}\right)^{n_{k+1}}\right)=\left(1+O\left(4^{-n_{k+1}}\right)\right),
$$

as desired.

Corollary 6.4. For all $k \geq 1, f$ has no zeros contained in $B_{k}$.

Next, we have the following estimates on the size of the coefficients $C_{k}$.

Lemma 6.5. Let $R>8, N>3$.

(1) When $k \geq 2$, we have $\left|C_{k}\right| \geq R_{k}^{n_{k-1}} / 2^{k} \geq 8 R_{k}$.

(2) When $k=1$, we have $\left|C_{1}\right|=R_{1}^{n_{1}} / 2 \geq 8 R_{1}$.

(3) For all $k \geq 1$, we have $\left|C_{k+1}\right| \geq\left|C_{k}\right|>1$.

Proof. The $k=1$ case is verified directly using Lemma 5.2 and the value of $C_{1}$ given in Lemma 6.1. For $k \geq 2$, we can compute, using the fact that $R_{j} \leq R_{k}^{1 / 2}$ whenever $j \leq k-1$, that

$$
\begin{aligned}
\left|C_{k}\right| & =\frac{1}{2^{k}} R_{k}^{n_{k}} \prod_{j=1}^{k-1} R_{j}^{-n_{j}} \geq \frac{1}{2^{k}} R_{k}^{n_{k}} \prod_{j=1}^{k-1} R_{k}^{-n_{j} / 2} \\
& =\frac{1}{2^{k}} R_{k}^{n_{k}-n_{k-1}}=\frac{1}{2^{k}} R_{k}^{n_{k-1}} .
\end{aligned}
$$

So in this case we see that it easily follows from Lemma 5.2 that

$$
\left|C_{k}\right| \geq \frac{1}{2^{k}} R_{k}^{n_{k-1}}>8 R_{k}
$$

Part (3) is easily checked by computing and estimating $\left|C_{k+1}\right| /\left|C_{k}\right|$; see Lemma 10.4 in Bis18.

The next two proofs are Lemmas 10.5 and 10.6 of Bis18. They are proved using Lemma 6.2 and factoring techniques similar to the proof of Lemma 6.3. They are quantitative statements that say that far enough away from the set of points where $\left|H_{m}(z)\right|=1, H_{m}$ looks like $z^{2 m}$ and near the origin, $H_{m}$ looks like $z^{m}$. Lemma 10.5 in Bis18 actually has an misprint in the statement with a missing factor of $R_{k}^{-n_{k}}$, although the proof is correct. The correct statements are below.

Lemma 6.6. For all $k \geq 1$, and for $z$ satisfying $5 R_{k} / 4 \leq|z| \leq 4 R_{k}$, we have

$$
f(z)=C_{k}\left(\frac{z}{R_{k}}\right)^{2 n_{k}}\left(1+O\left(\left(\frac{4}{5}\right)^{n_{k}}\right)\right)\left(1+O\left(R_{k}^{-1}\right)\right) .
$$


Lemma 6.7. For $k \geq 1$, and $R_{k} / 4 \leq|z| \leq 4 R_{k} / 5$, we have

$$
f(z)=2 C_{k}\left(\frac{z}{R_{k}}\right)^{n_{k}} \cdot\left(1+O\left(\left(\frac{4}{5}\right)^{n_{k}}\right)\right)\left(1+O\left(R_{k}^{-1}\right)\right) .
$$

We will see that most of the interesting mapping behavior for $f$ happens near $|z|=R_{k}$, where $f$ "interpolates" from being a perturbed degree $n_{k}$ power mapping to a perturbed degree $n_{k+1}$ power mapping.

The conformal mapping behavior of $f$ and its iterates will be very important later on, so we will need to control of the critical values and critical points of $f$. The statement below is Corollary 10.7 of [Bis18.

Theorem 6.8. For all $k \geq 1, f^{\prime}(z)$ is non-zero on $V_{k}$.

The proof of Theorem 6.8 requires Lemma 6.9

Lemma 6.9. Let $k \geq 1$. Then for all $z \in U_{k}$, there exists a holomorphic function $h_{k}(z)$ defined on $U_{k}$ such that

$$
f(z)=C_{k}\left(\frac{z}{R_{k}}\right)^{2 n_{k}}\left(1+h_{k}(z)\right) .
$$

The function $h_{k}(z)$ satisfies

$$
\left|h_{k}(z)\right|=O\left(\left(\frac{4}{5}\right)^{n_{k}}+R_{k}^{-1}\right) .
$$

Lemma 6.9 follows immediately from Lemma 6.6. For integers $k \geq 1$, define

$$
\epsilon_{k}=C\left(\left(\frac{4}{5}\right)^{n_{k}}+R_{k}^{-1}\right)
$$

The constant $C>0$ is chosen so that $\left|h_{k}(z)\right| \leq \epsilon_{k}$ on the annuli $U_{k}$. It follows that $\sum_{k=1}^{\infty} \epsilon_{k}$ can be made arbitrarily small, for $N$ and $R$ are sufficiently large. We state this carefully below.

Lemma 6.10. Let $C, h_{k}(z)$, and $\epsilon_{k}$ be defined as above. Let $\delta>0$ be given. Then for all $N$ and $R$ sufficiently large, we have

$$
\sum_{k=1}^{\infty} \epsilon_{k}<\delta
$$

Theorem 6.8 now follows from the Cauchy estimates applied to $h_{k}^{\prime}(z)$ and a direct estimate of $f^{\prime}(z)$ on $U_{k}$ using Lemma 6.9.

Lemma 6.11] is equation (10.5) on p. 435 of [Bis18], which contains a misprint due to the corresponding misprint of Lemma 10.5, and the proof is omitted. We include a corrected version along with a proof.

Lemma 6.11. For all $k \geq 1$ we have

$$
\frac{1}{4} \leq \frac{R_{k+1}}{\left|C_{k}\right| \cdot 2^{n_{k+1}}} \leq 4 .
$$

Proof. By (5.5), if $z \in A_{k}$ we have

$$
f(z)=f_{k}(z)\left(1+O\left(R_{k}^{-1}\right)\right) .
$$


Recall that $f_{k}$ was the $k$ th partial product of the infinite product defining $f$. By (5.1) it follows that if $R$ is sufficiently large, then

$$
\frac{1}{2} \leq \frac{M\left(f, 2 R_{k}\right)}{R_{k+1}} \leq 2
$$

Next note that Lemma 6.6 applies to $f$ on $|z|=2 R_{k}$ as well, and we also have

$$
\frac{1}{2} \leq \frac{M\left(f, 2 R_{k}\right)}{\left|C_{k}\right| \cdot 2^{n_{k+1}}} \leq 2 .
$$

The conclusion follows immediately.

We can now prove Theorem 6.12

Theorem 6.12. For all $N>10$ and for all sufficiently large $R$, we have that $A_{k+1} \subset f\left(V_{k}\right) \subset f\left(A_{k}\right)$ and $f\left(B_{k}\right) \subset B_{k+1}$. Moreover, $f$ maps the outer boundary component of $V_{k}$ into $B_{k+1}$ and the inner boundary component of $V_{k}$ into $B_{k}$.

For example, by Lemma 6.3 and Lemma 6.11, if $|z|=4 R_{k}$, we have

$$
|f(z)| \geq \frac{1}{4}\left|C_{k}\right| 4^{n_{k+1}} \geq \frac{1}{16} 2^{n_{k+1}} R_{k+1} .
$$

In a similar way, we can use Lemma 5.2 to see that if $|z|=4 R_{k}$

$$
|f(z)| \leq 4\left|C_{k}\right| 4^{n_{k+1}} \leq 16 \cdot 2^{n_{k+1}} R_{k+1} \leq \frac{16}{R_{k+1}^{2^{N}-1}} R_{k+2} .
$$

We can conclude that the outermost boundary component of $B_{k}$ is mapped well inside $B_{k+1}$ by $f$. Observations like this allow us to deduce Theorem 6.12, whose straightforward but somewhat tedious proof can be found in Sections 11 and 12 of Bis18.

As a result we can obtain our first dynamical corollaries.

Corollary 6.13. For every $k \geq 1, B_{k}$ is in the Fatou set of $f$.

Proof. Since $f\left(B_{k}\right) \subset B_{k+1}$, we know that if $z \in B_{k}$, then the iterates $f^{n}(z)$ tend to infinity locally uniformly.

Recall that an asymptotic value of an entire function $g$ is a point $w \in \mathbb{C}$ such that there exists a curve $\gamma:[0, \infty) \rightarrow \mathbb{C}$ such that $\gamma(t) \rightarrow \infty$ and $g(\gamma(t)) \rightarrow w$ as $t \rightarrow \infty$.

Corollary 6.14. $f$ has no finite asymptotic values.

Proof. If $\gamma:[0, \infty) \rightarrow \mathbb{C}$ satisfies $\gamma(t) \rightarrow \infty$ as $t \rightarrow \infty$, then there exists a sequence $\left\{t_{k}\right\}_{k=1}^{\infty}$ with $t_{k}<t_{k+1}, t_{k} \rightarrow \infty$ and $\gamma\left(t_{k}\right) \in B_{k}$. Therefore by Theorem 6.12 $f\left(\gamma\left(t_{k}\right)\right) \in B_{k+1}$, so that $f\left(\gamma\left(t_{k}\right)\right) \rightarrow \infty$. Therefore $f$ has no finite asymptotic values.

\section{BeHAVIOR OF $f$ NEAR THE ORIGIN}

Having analyzed the behavior of $f$ away from the origin, we now analyze $f$ near the origin. The primary goal of this section is to show that the Fatou set of $f$ contains an attracting basin containing the origin, and that the boundary of this fractal basin of attraction moves holomorphically with respect to some suitable one-parameter family of polynomial-like mappings. This will allow us to control the Hausdorff dimension of the boundary of the basin of attraction. 
We begin with a general discussion about holomorphic perturbations of the polynomial $g^{N}(z)=\left(z^{2}+c\right)^{\circ N}$. In this section, we will assume that $R>100$, define $r=100$ and define $B=B(0, r)$. With this notation, $\left(g^{N},\left(g^{N}\right)^{-1}(B), B\right)$ is a degree $2^{N}$ polynomial-like mapping. The Julia set and filled Julia set of the polynomiallike mapping $\left(g^{N},\left(g^{N}\right)^{-1}(B), B\right)$ are the same as the Julia set and filled Julia set of the polynomial $g^{N}(z)$, respectively. $g^{N}$ is also hyperbolic on its quasicircle Julia set. If we fix a value $\mu \in(1,2)$ and choose some value of $N$ sufficiently large, this implies that there exists a Jordan annulus $A$ containing $\mathcal{J}\left(g^{N}\right)$ so that

$$
\left|\left(g^{N}\right)^{\prime}(z)\right| \geq \mu>1,
$$

for all $z \in A$. The value of $N$ depends only on $\mu$ and the value $c$ defining $g^{N}(z)$.

Lemma 7.1. Let $\mu$ be as defined in (7.1) and let $\epsilon(z): B(0,2 r) \rightarrow \mathbb{C}$ be a holomorphic function. Then for every $\eta<\frac{\mu-1}{2}$, there exists a constant $\delta>0$ so that if $|\epsilon(z)| \leq \delta$ for all $z \in B(0,2 r)$, then the function $h(z)=g^{N}(z)(1+\epsilon(z))$ satisfies

$$
\sup _{z \in B(0, r)}\left|h^{\prime}(z)-\left(g^{N}\right)^{\prime}(z)\right|<\eta
$$

and

$$
\left|h^{\prime}(z)\right| \geq \mu_{0}=\left(\frac{1+\mu}{2}\right)>1,
$$

for all $z \in A$.

Proof. Let $K=\sup _{z \in B(0,2 r)}\left|g^{N}(z)\right|$ and $K^{\prime}=\sup _{z \in B(0, r)}\left|\left(g^{N}\right)^{\prime}(z)\right|$. By the Cauchy estimates,

$$
\max _{z \in B(0, r)}\left|\epsilon^{\prime}(z)\right| \leq \frac{\delta}{r}
$$

Therefore,

$$
\begin{aligned}
\sup _{z \in B(0, r)}\left|h^{\prime}(z)-\left(g^{N}\right)^{\prime}(z)\right| & =\sup _{z \in B(0, r)}\left|\left(g^{N}\right)^{\prime}(z) \epsilon(z)+g^{N}(z) \epsilon^{\prime}(z)\right| \\
& \leq K^{\prime} \cdot \delta+K \cdot \frac{\delta}{r} \\
& =O(\delta) .
\end{aligned}
$$

Therefore, for sufficiently small $\delta$, (7.2) holds, and (7.3) follows immediately.

Remark 7.2. An analysis of the proof of Lemma 7.1 shows that $\delta$ depends on the fixed value $c$, the fixed value $r$, the value $\eta$ in (7.2) and the positive integer $N$.

Lemma 7.3. Fix a positive integer $N$, let $\mu$ be defined as in (17.1), and let $\eta<$ $(\mu-1) / 2$ be given. Choose $\delta>0$ so that Lemma 7.1 holds for $h(z)=g^{N}(z)(1+\epsilon(z))$ for the given values $\eta$ and $N$. Define $U=h^{-1}(B)$. Then $(h, U, B)$ is degree $2^{N}$ polynomial-like mapping.

Proof. By perhaps choosing $\delta$ smaller, we conclude that $h^{-1}(A)$ and $\left(g^{N}\right)^{-1}(A)$ are Jordan annuli compactly contained in $A$. By (7.3), $h$ has no critical points in $h^{-1}(A)$, and by (7.2), $h$ has no critical points in the unbounded complementary component of $h^{-1}(A)$. Therefore, all critical points of $h$ are contained in the bounded complementary component of $h^{-1}(A)$. Let $D$ denote the bounded complementary component of $h^{-1}(A)$. Then by (7.3) we know $h$ expands $h^{-1}(A)$ onto $A$ and we have $\overline{(h(D))} \subset D$, so the critical values of $h$ are also contained in $D$. It follows that $(h, U, B)$ is a degree $2^{N}$ polynomial-like mapping. 
For the rest of the section, we will assume that $\delta$ is small enough so that conclusions Lemma 7.1 and Lemma 7.3 hold.

Lemma 7.4. For all $z \in B\left(0, \frac{1}{4} R_{1}\right)$, we have

$$
f(z)=g^{N}(z)\left(1+O\left(4^{-n_{1}}\right)\right)\left(1+O\left(R_{2}^{-1}\right)\right) .
$$

Assume that $R$ is large enough so that $R_{1}^{1 / 2}<\frac{1}{4} R_{1}$. Then by perhaps choosing $R$ larger, for all $z \in B\left(0, R_{1}^{1 / n_{2}}\right)$ we also have

$$
f(z)=g^{N}(z) \cdot\left(1+O\left(R_{1}^{\frac{1}{2}-n_{1}}\right)\right) .
$$

Proof. The techniques are similar to the previous section. Indeed, when $|z| \leq R_{1} / 4$ we have by (5.5) that

$$
f(z)=g^{N}(z)\left(1-\frac{1}{2}\left(\frac{z}{R_{1}}\right)^{n_{1}}\right) \prod_{k=2}^{\infty} F_{k}(z)=g^{N}(z)\left(1+O\left(4^{-n_{1}}\right)\right)\left(1+O\left(R_{2}^{-1}\right)\right) .
$$

The proof of (7.5) is similar.

When $z \in B\left(0, R_{1}^{1 / n_{2}}\right)$ we will write $f(z)=g^{N}(z) \cdot(1+\epsilon(z))$, where $|\epsilon(z)|=$ $O\left(R_{1}^{\frac{1}{2}-n_{1}}\right)$ as $R \rightarrow \infty$ by (7.5). It follows that may choose $R$ sufficiently large so that $|\epsilon(z)|<\delta$ for all $z \in B(0, r)$. We define

$$
t:=\sup _{z \in B(0, r)}|\epsilon(z)| .
$$

We remark that $t \rightarrow 0$ as $R \rightarrow \infty$.

For the rest of this section, we will study the following one parameter family of functions. Fix $R$ so that (7.6) holds for $t<\delta$. Let $\lambda \in \mathbb{D}$ and define

$$
F_{\lambda}(z):=g^{N}(z)\left(1+\lambda \cdot \frac{\epsilon(z)}{t}\right) .
$$

Note that $F_{0}(z)=g^{N}(z)$. We also remark that when $|\lambda|=1$, we have $\left|t^{-1} \epsilon(z)\right| \leq \delta$ for all $z \in B(0, r)$. When $\lambda=t$, we have $F_{t}(z)=f(z)$.

Definition 7.5. A holomorphic motion of a set $E \subset \mathbb{C}$ is a family of injective maps

$$
\varphi_{\lambda}: E \rightarrow \mathbb{C}
$$

where $\lambda \in \mathbb{D}$, such that $\lambda \mapsto \varphi_{\lambda}(z)$ is holomorphic for fixed $z \in E$, and $\varphi_{0}$ is the identity.

For the rest of the section, we will define $F_{\lambda}^{-1}(B)=U_{\lambda}$. Our goal is to show that the Julia sets $J_{F_{\lambda}}$ of the polynomial-like mappings $\left(F_{\lambda}, U_{\lambda}, B\right)$ move holomorphically. That is, we want a holomorphic motion that satisfies

$$
\varphi_{\lambda}: J_{F_{0}} \rightarrow J_{F_{\lambda}},
$$

and satisfies the equivariance condition

$$
F_{\lambda} \circ \varphi_{\lambda}(z)=\varphi_{\lambda} \circ F_{0}(z) .
$$

We need the following two fundamental facts about holomorphic motions. First, we have the $\lambda$-lemma (Theorem 4.1 of [McM94]). 
Theorem 7.6 (The $\lambda$-lemma). A holomorphic motion of a set $E$ has a unique extension to a holomorphic motion of $\bar{E}$. The extended motion is a continuous mapping $\varphi: \mathbb{D} \times \bar{E} \rightarrow \mathbb{C}$ for $\varphi(\lambda, z):=\varphi_{\lambda}(z)$. For each $\lambda \in \mathbb{D}, \varphi_{\lambda}$ extends to a quasiconformal map of $\mathbb{C}$ to $\mathbb{C}$.

The $\lambda$-lemma implies that to show the Julia sets of the polynomial-like mappings $\left(F_{\lambda}, U_{\lambda}, B\right)$ move holomorphically, it suffices to construct the holomorphic motion of the repelling periodic points, since these points are dense in the Julia set. Next, the following result of Bers and Royden, [BR86], shows that the dilatation of holomorphic motions can be controlled in the following precise way:

Theorem 7.7. If $\varphi: \mathbb{D} \times E \rightarrow \mathbb{C}$ for $\varphi(\lambda, z):=\varphi_{\lambda}(z)$ is a holomorphic motion, then for all $\lambda \in \mathbb{D}, \varphi_{\lambda}$ is the restriction to $E$ of a $K$-quasiconformal map $H_{\lambda}: \mathbb{C} \rightarrow \mathbb{C}$, where

$$
K \leq \frac{1+|\lambda|}{1-|\lambda|}
$$

Finally, we will use the following version of the holomorphic implicit function theorem (see, for example, Var11, Theorem 2.3.10).

Theorem 7.8. Let $U \subset \mathbb{C} \times \mathbb{C}$ be open with $\left(z_{0}, w_{0}\right) \in U$. Let $F: U \rightarrow \mathbb{C}$ be continuous and holomorphic in each variable separately. Suppose that $F\left(z_{0}, w_{0}\right)=0$ and $\frac{\partial F}{\partial z}\left(z_{0}, w_{0}\right) \neq 0 . \quad$ Let $\Pi_{2}: \mathbb{C} \times \mathbb{C} \rightarrow \mathbb{C}$ denote projection onto the second coordinate. Then there exists an open set $V$ containing $\left(z_{0}, w_{0}\right)$ so that for all $w \in \Pi_{2}(V)$, the equation $F(z, w)=0$ has a unique solution $z=\varphi(w)$ so that $(\varphi(w), w) \in V$. Moreover, the function $w \mapsto \varphi(w)$ is holomorphic.

Now we move onto the family of functions $F_{\lambda}$.

Lemma 7.9. For each $\lambda \in \mathbb{D}$, the Julia set of $\left(F_{\lambda}, U_{\lambda}, B\right)$ is the closure of exactly one basin of attraction.

Proof. By Lemma 7.1, we have $\left|F_{\lambda}^{\prime}\right|>\mu_{0}>1$ on the Jordan annulus $A$ (defined via (7.1)) for all $\lambda \in \mathbb{D}$. Let $D_{\lambda}$ be the bounded complementary component of the Jordan annulus $F_{\lambda}^{-1}(A)$. Then $\overline{F_{\lambda}\left(D_{\lambda}\right)} \subset D_{\lambda}$, and there exists a unique attracting fixed point $c_{\lambda} \in D_{\lambda}$ for $F_{\lambda}$. This fixed point is the only possible fixed point or periodic cycle contained in $D_{\lambda}$.

Any other periodic cycles for $\left(F_{\lambda}, U_{\lambda}, B\right)$ must be contained in $A$. Since $\left|F_{\lambda}^{\prime}\right|>$ $\mu_{0}>1$ on $A$, such a cycle must be repelling. Therefore, $F_{\lambda}$ has exactly one attracting fixed point, and all other periodic cycles are repelling. It follows that $K_{F_{\lambda}}$, the filled Julia set of $\left(F_{\lambda}, U_{\lambda}, B\right)$, is the closure of exactly one basin of attraction.

We will denote the basin of attraction of $\left(F_{\lambda}, U_{\lambda}, B\right)$ by $B_{F_{\lambda}}$. Note that $\partial B_{F_{\lambda}}=$ $J_{F_{\lambda}} \subset A$.

Lemma 7.10. The repelling periodic cycles of $\left(F_{\lambda}, U_{\lambda}, B\right)$ depend holomorphically on $\lambda \in \mathbb{D}$.

Proof. For integers $m \geq 1$, we study the equation

$$
Q(z, \lambda)=F_{\lambda}^{m}(z)-z=0 .
$$

The solutions to this equation are exactly the periodic points of $F_{\lambda}$ of period dividing $m$. Let $z_{0}$ be a repelling periodic point of period $m$ for $F_{0}$, and note that 
$z_{0}$ must remain in $A$ for all iterates. Let $\nu$ be the multiplier of $F_{0}$ at $z_{0}$. Then $z_{0} \in J_{F_{0}},|\nu| \geq \mu_{0}>1$, and

$$
\left|\frac{\partial Q}{\partial z}\left(z_{0}, 0\right)\right|=|\nu-1|>0 .
$$

By the holomorphic implicit function theorem, the periodic point $z_{0}$ moves holomorphically. Since the filled Julia set of $\left(F_{\lambda}, U_{\lambda}, B\right)$ is the closure of exactly one basin of attraction, the point remains repelling as we vary $\lambda$.

For a repelling periodic point $z$ of $F_{0}$ we define $h_{\lambda}(z)$ to be the corresponding holomorphic motion of that point. If $E$ is the set of all repelling periodic cycles of $F_{0}$, this defines a mapping

$$
h_{\lambda}: \mathbb{D} \times E \rightarrow E_{\lambda},
$$

where $E_{\lambda}$ is the set of repelling periodic cycles for $\left(F_{\lambda}, U_{\lambda}, B\right)$.

Lemma 7.11. The mapping $h: \mathbb{D} \times E \rightarrow \mathbb{C}$ for $h(\lambda, z)=h_{\lambda}(z)$ is a holomorphic motion.

Proof. It only remains to check injectivity. Again, for some given $\lambda$, a periodic point $z_{\lambda}$ of period diving $m$ is a solution of

$$
Q(z, \lambda)=F_{\lambda}^{m}(z)-z=0 .
$$

By the implicit function theorem, there exists a neighborhood of $\left(z_{\lambda}, \lambda\right)$ such that for all choices of $\lambda^{\prime}$ in this neighborhood there is a unique $z_{\lambda^{\prime}}$ so that $Q\left(z_{\lambda^{\prime}}, \lambda^{\prime}\right)=0$. So by the uniqueness statement of the implicit function theorem, we must have injectivity.

Corollary 7.12. The Julia sets of $\left(F_{\lambda}, U_{\lambda}, B\right)$ move holomorphically.

Proof. By the $\lambda$-lemma, the holomorphic motion $h_{\lambda}$ extends to the closures of the repelling periodic points. We just need to check that (7.8) holds for all $z \in J_{F_{0}}$. Since $h_{\lambda}$ maps periodic points of period $m$ onto distinct periodic points of period $m$, if $z$ is periodic with period $m$, we have

$$
h_{\lambda}\left(F_{0}^{m}(z)\right)=h_{\lambda}(z)=F_{\lambda}^{m}\left(h_{\lambda}(z)\right) .
$$

It follows that $F_{\lambda}\left(\left(h_{\lambda}\right)(z)\right)$ and $h_{\lambda}\left(F_{0}(z)\right)$ must belong to the same periodic cycle and that they must be equal. Therefore, (7.8) holds on the repelling periodic points of $J_{F_{0}}$, and by density of the repelling periodic points, (7.8) extends to hold on the associated Julia sets of the polynomial-like mappings.

Corollary 7.13. For each $\lambda \in \mathbb{D}$, the Julia set of $\left(F_{\lambda}, U_{\lambda}, B\right)$ is a quasicircle which coincides with $\partial B_{F_{\lambda}}$.

Proof. The Julia set of $\left(F_{0}, U_{0}, B\right)$ coincides with $\mathcal{J}\left(g^{N}\right)$, which is a quasicircle. The result now follows from Corollary 7.12 and the $\lambda$-Lemma, since quasiconformal mappings map quasicircles to quasicircles.

Corollary 7.14. We have

$$
\lim _{|\lambda| \rightarrow 0} \operatorname{dim}_{H}\left(J_{F_{\lambda}}\right)=\operatorname{dim}_{H}\left(J_{F_{0}}\right) .
$$


Proof. Theorem 7.7 shows that the $h_{\lambda}$ extends to a $K$-quasiconformal self map of $\mathbb{C}$, where $K \leq \frac{1+|\lambda|}{1-|\lambda|}$. Since $K$-quasiconformal mappings are locally $1 / K$-Holder continuous, for $\lambda \in \mathbb{D}$ we have

$$
\frac{1-|\lambda|}{1+|\lambda|} \operatorname{dim}_{H}\left(J_{F_{0}}\right) \leq \operatorname{dim}_{H}\left(J_{F_{\lambda}}\right) \leq \frac{1+|\lambda|}{1-|\lambda|} \operatorname{dim}_{H}\left(J_{F_{0}}\right) .
$$

Equation (7.9) follows immediately.

Lemma 7.15. Let $t$ be defined as in (7.6). Then we have the inclusion $J_{F_{t}} \subset \mathcal{J}(f)$. In other words, the Julia set of the polynomial-like mapping $\left(F_{t}, U_{\lambda}, B\right)$ is a subset of the Julia set of the entire function $f$.

Proof. The repelling periodic cycles for $\left(F_{t}, U_{t}, B\right)$ are by definition also repelling periodic cycles for the entire function $f(z)$. The inclusion $J_{F_{t}} \subset \mathcal{J}(f)$ follows immediately.

Suppose that $s=\operatorname{dim}_{\mathrm{H}}\left(\mathcal{J}\left(g^{N}\right)\right)$ and $\epsilon_{0}>0$ is given. It follows from Corollary 7.14 and Lemma 7.15 that for all sufficiently large values of $R$, we have

$$
s-\epsilon_{0} \leq \operatorname{dim}_{\mathrm{H}}(\mathcal{J}(f)) \leq s+\epsilon_{0} .
$$

\section{Conformal mapping properties of the Fatou and Julia set}

Let $k \geq 0$. Define,

$$
\begin{gathered}
A_{0}:=\left\{|z| \leq \frac{R_{1}}{4}: f(z) \in A_{1}\right\}, \\
A_{-k}:=\left\{|z| \leq \frac{R_{1}}{4}:\left|f^{j}(z)\right| \leq \frac{R_{1}}{4}, j=1,2, \ldots, k, \text { and } f^{k+1}(z) \in A_{1}\right\} .
\end{gathered}
$$

After $k+1$ iterates, $f$ maps $A_{-k}$ into $A_{1}$. We make similar definitions for $V_{k}, U_{k}$, and $B_{k}, k \leq 0$. While these definitions are the same as in Bis18, it is important to note a key difference for the functions we consider is that for each $k \leq 0, A_{k}$, $V_{k}, U_{k}$, and $B_{k}$ consist of exactly one connected component. This is because the functions considered in Bis18 have critical points near the origin which escape under iteration; the functions we consider have critical points near the origin that remain in a basin of attraction. See Lemma 8.1 and Lemma 8.4 .

Let $\mathcal{C}$ denote the set of all points which eventually get mapped into the filled Julia set $K_{f}$ of $f$ viewed as a polynomial-like mapping. Then

$$
\mathcal{C}=\bigcup_{k=1}^{\infty} f^{-k}\left(K_{f}\right)
$$

Now define

$$
A:=\bigcup_{k \in \mathbb{Z}} A_{k}
$$

It follows from Theorem 6.12 that $f^{-1}(A) \subset A$.

The first lemma of this section characterizes the dynamics of the critical points of $f$ contained in $A_{k}$ for $k \geq 1$. We denote $B_{f}$ as the basin of attraction for $f$ when viewed as a polynomial-like mapping. Since $f$ has no asymptotic values, it's postcritical set is $P(f)=\left\{f^{n}(z): n \geq 1, z\right.$ a critical point $\}$. 
Lemma 8.1. For sufficiently large $R$, the critical points of $f$ are either contained in $B_{f}$ or $A_{k}$ for some $k \geq 1$. If $z$ is a critical point contained in $A_{k}$, then $f(z) \in B_{k}$. In both cases, $z$ is in the Fatou set.

This lemma is proved by studying the size of $\left|H_{n_{k}}\right|$ at a critical point of $f$. Since $f$ is close to a multiple of $H_{n_{k}}$ on $A_{k}$, the critical points of $f$ contained in $A_{k}$ are very close to the critical points of $H_{n_{k}}$. However, $\left|H_{n_{k}}\right|=1$ at any of its critical points, so we expect that $H_{n_{k}}$ should have modulus close to 1 at a critical point for $f$. This calculation is done explicitly in Lemma 14.2 in Bis18, where at a critical point it is shown that

$$
\left|1-H_{n_{k}}(z)\right| \leq n_{k}^{-2}
$$

It follows from Lemma 6.2 and Lemma 6.5 that the distance between a critical value and the circle $C\left(0,4 R_{k}\right)$ is approximately

$$
\left|C_{k}\right|-4 R_{k} \geq R_{k}^{n_{k-1}} / 2^{k}-4 R_{k},
$$

when $k \geq 2$ and approximately

$$
R_{1}^{n_{1}} / 2-4 R_{1}
$$

when $k=1$. It follows that the distance between $P(f)$ and $\mathcal{J}(f)$ is strictly larger than 0 .

Lemma 8.2 of this section tells us where the Julia set is located in each $A_{k}$, $k \geq 1$. Recall that $\Omega_{n_{k}}^{p}$ is a "petal" region where $H_{n_{k}}(z)=z^{n_{k}}\left(2-z^{n_{k}}\right)$ restricts to a conformal mapping onto the disk. Let $\mathcal{P}_{n_{k}}$ denote the union of all the petals of $H_{n_{k}}$. Then $R_{k} \cdot \Omega_{n_{k}}^{p}$ is a petal of $H_{n_{k}}\left(z / R_{k}\right)$, so we will let $R_{k} \cdot \mathcal{P}_{n_{k}}$ denote the set of all $n_{k}$ petals of $H_{n_{k}}\left(z / R_{k}\right)$.

Lemma 8.2. Let $k \geq 1$. Then $\left(\mathcal{J}(f) \cap A_{k}\right) \subset V_{k} \cup\left(R_{k} \cdot \mathcal{P}_{n_{k}}\right)$. Let $R_{k} \cdot \Omega_{n_{k}}^{p}$ be $a$ petal. Then

$$
\operatorname{diam}\left(\mathcal{J}(f) \cap R_{k} \cdot \Omega_{n_{k}}^{p}\right)=O\left(R_{k}^{-2}\right) .
$$

To prove this lemma, one uses Lemma 6.6 to conclude that for all $k \geq 1$, the closed annulus $\left\{z: 5 R_{k} / 2 \leq|z| \leq 4 R_{k}\right\}$ gets mapped by $f$ into $B_{k+1}$, and then similarly uses Lemma 6.7 to show that for all $k \geq 1,\left\{z: R_{k} / 4 \leq|z| \leq 4 R_{k} / 5\right\}$ gets mapped by $f$ into $B_{k}$. It follows from Corollary 6.13 that these closed annuli belong in the Fatou set of $f$. To deal with the points in $\left\{z: 4 R_{k} / 5 \leq|z| \leq 3 R_{k} / 2\right\}$, we first observe that the zeros of $f$ in $A_{k}$ belong to this sub-annulus, and for any $\eta \in(0,1 / 2)$ we define

$$
T_{k}^{\eta}=\left\{z: 4 R_{k} / 5 \leq|z| \leq 3 R_{k} / 2 \text { and }\left|H_{n_{k}}\left(z / R_{k}\right)\right|>\eta\right\} .
$$

The points where $4 R_{k} / 5 \leq|z| \leq 3 R_{k} / 2$ and $\left|H_{n_{k}}\left(z / R_{k}\right)\right| \leq \eta$ are contained in $R_{n_{k}} \cdot \mathcal{P}_{n_{k}}$. One can show that for all $k \geq 1$, if $\eta=R_{k}^{-3}$, then we must have $f\left(T_{k}^{\eta}\right) \subset B_{k}$, so that $T_{k}^{\eta}$ is in the Fatou set. The conclusion of Lemma 8.2 now follows, and equation (8.5) follows from Lemma 3.2 and (8.2). The details for all of these arguments follow similarly as in Sections 11-13 of [Bis18].

Recall that for a set $A, \widehat{A}$ denotes the union of $A$ and all its bounded complementary components. Lemma 8.1 implies the following.

Lemma 8.3. For an integer $k$, let $D_{k-1}$ denote the connected component of $f^{-1}\left(\widehat{A}_{k}\right)$ containing the origin. Then when $k \geq 1$,

$$
f: D_{k-1} \rightarrow \widehat{A}_{k}
$$


is a degree $n_{k}$ branched covering map. When $k \leq 0$, then

$$
f: D_{k-1} \rightarrow \widehat{A}_{k}
$$

is a degree $2^{N}$ branched covering map.

The basic covering map lemma above can be refined as follows:

Lemma 8.4. Let $W$ be a connected component of $f^{-1}\left(A_{k}\right)$ for $k \in \mathbb{Z}$. Then we have the following possibilities.

(1) For $k \geq 1$, we have $W \subset V_{k-1}$, and $f: W \rightarrow A_{k}$ is a degree $n_{k}$ covering map. For $k \leq 0$, we have $W=A_{k-1}$ and $f: W \rightarrow A_{k}$ is a degree $2^{N}$ covering map.

(2) There exists some $j \geq \max \{1, k\}$ so that $W \subset A_{j}$, and $W$ is contained in some petal in $R_{j} \cdot \mathcal{P}_{n_{j}}$. Conversely, for all such $j$ and every petal $R_{n_{j}} \cdot \Omega_{n_{j}} \in$ $R_{n_{j}} \cdot \mathcal{P}_{n_{j}}$, there exists exactly one component $W=f^{-1}\left(A_{k}\right) \subset R_{n_{j}} \cdot \Omega_{n_{j}}$. In both cases, $f: W \rightarrow A_{k}$ is a conformal mapping.

Moreover, these are the only possibilities.

Proof. (1) By Theorem 6.12, a component $W$ certainly exists. When $k \geq 1$, by Lemma 6.9 and Lemma 8.1 each point $z \in A_{k}$ is evenly covered; there is a ball $B(z, r)$ so that $f^{-1}(B(z, r)) \cap W$ is the disjoint union of $n_{k}$ simply connected topological disks in $W$, and $f$ is conformal on each of these disks. When $k \leq 0$, we argue in a similar way using Lemma 7.4 and Lemma 8.1 .

(2) The fact that all possible $j \geq k$ occurs follows from the fact that all the zeros of $f$ are in the annuli $A_{j}$ for $j \geq 1$, and the fact that $f$ is continuous. $W$ must also lie completely in some petal region. Indeed, by Lemma 8.2, $W$ must contain elements of the Julia set, but if $W \subset V_{j}$, then $f(W)$ could not be a subset of $A_{k}$ by Lemma 6.12. So $W$ must be strict subset of a petal and cannot intersect the boundary of a petal, or else the proof of Lemma 8.2 would show that there are points in $W$ that map to $B_{k}$. The fact that there is at least one component per petal again follows from the continuity of $f$.

It remains to show the desired conformal mapping behavior occurs. Let $z \in A_{k}$, and view $f$ as a mapping $f: W \rightarrow A_{k}$. Again Lemma 8.1 shows that each $z$ in $A_{k}$ is evenly covered. We want to further show that $f$ is actually one-to-one on $W$. To do this, notice that when we view $f: \mathbb{C} \rightarrow \mathbb{C}, z$ has $n_{k}$ many preimages in $V_{k-1}$, and $z$ has at least $n_{l}$ many preimages in each $A_{l}$ (one for each petal), for $l=k, k+1, \ldots, j$. This gives

$$
n_{k}+\left(n_{k}+\cdots+n_{j}\right)=n_{j+1}
$$

total preimages in $\widehat{A}_{j}$. But $f: D_{j} \rightarrow \widehat{A}_{j+1}$ is a degree $n_{j+1}$ branched covering map, so $f: W \rightarrow A_{k}$ must be one-to-one.

We would like to remark on the following important consequence. If $W \subset A_{j}$ is a component of $f^{-1}\left(A_{k}\right)$ for $j \geq k$, then $f: \widehat{W} \rightarrow \widehat{A}_{k}$ is conformal. Since $f$ is injective on the Jordan annulus $W$, it follows from the argument principle that it is injective on $\widehat{W}$.

Let $W \subset A_{j}$ be a component of $f^{-1}\left(A_{k}\right)$ for $j \geq k$. $f: W \rightarrow A_{k}$ is conformal, and the distortion of this conformal mapping can be controlled to be as small as we would like. If $B=B(z, r)$ is a ball of radius $r$, we denote $\lambda B=B(z, \lambda r)$. 
Lemma 8.5. Let $\lambda>2$ be given and let $W$ be as above. Then for all sufficiently large choices of $R$, there exists a ball $B=B(z, r)$ containing $W$ so that $f$ restricted to $\lambda B$ is conformal.

Proof. Let $\left\{z_{1}, \ldots, z_{n_{k}}\right\}$ denote the critical points of $f$ contained in $A_{k}$ and let $\left\{f\left(z_{1}\right), \ldots, f\left(z_{n_{k}}\right)\right\}$ denote the corresponding critical values. Let

$$
\rho_{k}=\min \left\{\left|f\left(z_{1}\right)\right|, \ldots,\left|f\left(z_{n_{k}}\right)\right|\right\} .
$$

By (8.3) and (8.4), for $R$ sufficiently large, we can make the ratio $\rho_{k} / R_{k}$ as large as we would like, independent of $k$. Let $A_{k}^{*}$ denote the annulus $\left\{z: \frac{1}{4} R_{k} \leq|z| \leq \frac{1}{2} \rho_{k}\right\}$.

Let $W^{*}$ be the connected component of $f^{-1}\left(A_{k}^{*}\right)$ containing $W$. Then as we argued above, $f: W^{*} \rightarrow A_{k}^{*}$ is a conformal mapping. By Lemma 8.2, there exists a ball $B(z, r)$ containing $W$ with $\operatorname{diam}(B(z, r))=O\left(R_{k}^{-2}\right)$. The modulus of $W^{*} \backslash$ $B(z, r)$ can be made as large as we would like, independent of $k$, so for sufficiently large $R$ it follows that $\lambda B(z, r) \subset W^{*}$ as well.

\section{9. $C^{1}$ BOUNDARY COMPONENTS}

The purpose of this section is to show that the boundaries of Fatou components of $f$ are $C^{1}$, and in many cases these boundary components are close to being circles.

Let $\epsilon>0$. We will say a set $C$ is an $\epsilon$-approximate circle if, for some translation $C^{\prime}$ of $C$, there exists a circle $C(0, r)$ and a mapping $h: C(0, r) \rightarrow C^{\prime}$ which has the form $h\left(r e^{i \theta}\right)=(r(\theta), \theta)$ in polar coordinates where $r:[0,2 \pi] \rightarrow \mathbb{R}$ is $\epsilon$-Lipschitz. We record the following geometric lemma for future reference, which follows from the definition above and (3.2) of Lemma 3.2.

Lemma 9.1. Let $0<r^{\prime}<r$ be given. Let $\Omega$ be a Jordan domain and suppose that $f: B(0, r) \rightarrow \Omega$ is conformal. Suppose that $C \subset B\left(0, r^{\prime}\right)$ is an $\eta$-approximate circle. Then there exists a constant $\mu>0$ such that if $r / r^{\prime}>\mu$, then $f(C)$ is an $\epsilon$-approximate circle, where $|\epsilon-\eta|$ depends only on $\mu$. Moreover, as $r / r^{\prime} \rightarrow \infty$, $|\epsilon-\eta| \rightarrow 0$.

Let $\Gamma$ be a Jordan curve in the plane, with $\gamma:[0,1] \rightarrow \Gamma$ as its parameterization. We say that $\Gamma$ has a tangent at $z_{0}=\gamma\left(t_{0}\right)$ if

$$
\lim _{t \rightarrow t_{0}^{+}} \frac{\gamma(t)-\gamma\left(t_{0}\right)}{\left|\gamma(t)-\gamma\left(t_{0}\right)\right|}=e^{i \tau}
$$

and

$$
\lim _{t \rightarrow t_{0}^{-}} \frac{\gamma(t)-\gamma\left(t_{0}\right)}{\left|\gamma(t)-\gamma\left(t_{0}\right)\right|}=-e^{i \tau}
$$

We say $\Gamma$ has a unit tangent vector $e^{i \tau}$ at $z_{0}$. We say that $\Gamma$ is a $C^{1}$-smooth curve if it has a tangent at each point $z \in \Gamma$ if if the unit tangent vector changes continuously with $z \in \Gamma$.

We will need Lemma 9.2, which is Lemma 18.1 in Bis18.

Lemma 9.2. Suppose $h$ is a holomorphic function on $U=\{z: 1<|z|<4\}$ and suppose that $|h|$ is bounded by $\epsilon$ on $U$. Let $H(z)=z^{m}(1+h(z))$. For any fixed $\theta$ the radial segment $S(\theta)=\left\{r e^{i \theta}: 3 / 2 \leq r \leq 5 / 2\right\}$ is mapped by $H$ to a curve that makes angle at most $O(\epsilon / m)$ with any radial ray it meets. 
The lemma is proved by applying the Cauchy estimate to $z \frac{H^{\prime}(z)}{H(z)}$; the argument of this expression measures the angle. Lemma 9.2 also implies the following stronger result. If $\Gamma$ is an analytic Jordan arc in $U$, and $\tau_{z}$ is the unit tangent vector to $\Gamma$ at $z$, then the difference between the angle between $\tau_{z}$ and $S(\theta)$ and the angle between $\tau_{f(z)}$ and any ray it meets is $O(\epsilon / m)$. This follows immediately from the angle preserving property of holomorphic maps with non-zero derivative combined with Lemma 9.2 .

Recall that by Theorem 6.12 for all $k \geq 1$ we have $A_{k+1} \subset f\left(V_{k}\right)$. It follows from Lemma 9.2 and Lemma 8.1 that $W=f^{-1}\left(V_{k+1}\right) \subset V_{k}$ is a topological annulus, and the boundary components of $W$ are $O\left(\epsilon_{k}\right)$-approximate circles, where $\epsilon_{k}$ for $k \geq 1$ was defined in (6.3). From here, with the additional help of Lemma 6.6, we can also deduce that the width of $W$ is approximately $R_{k} / 2 n_{k}$. It turns out that this contracting, small angle distorting behavior is precisely what we need to prove the following theorem. See also Section 18 of [Bis18.

Theorem 9.3. Let $\epsilon>0$ be given. Then there exists $R$ sufficiently large so that for all $k \geq 1$, there exists a $C^{1}$-smooth, $\epsilon$-approximate circle $\Gamma_{k} \subset V_{k}$ contained in $\mathcal{J}(f)$ that surrounds the origin.

Proof. Fix some $k \geq 1$ and define

$$
\Gamma_{k, n}=\left\{z \in V_{k}: f^{j}(z) \in A_{k+j}, j=1, \ldots, n\right\} .
$$

Since $A_{k+n}$ is a round annulus, it has a natural foliation $\mathcal{U}_{k+n}$ of closed circles centered at the origin. $\Gamma_{k, n}$ has an induced foliation $\mathcal{U}_{k+n}^{\prime}$ of closed analytic Jordan curves obtained by pulling back each element of $\mathcal{U}_{k+n}$ by $f^{n}$. The fact that these curves are indeed Jordan curves follows from Lemma 8.1 and the covering map properties of $f^{n}: \Gamma_{k, n} \rightarrow A_{k+n}$.

Define $\Gamma_{k}=\bigcap_{n=1}^{\infty} \Gamma_{k, n}$. We claim that the compact connected set $\Gamma_{k}$ satisfies the conditions of the theorem. We first produce candidate tangents for each $z \in \Gamma_{k}$. For each $z \in \Gamma_{k}$, let $\gamma_{k, n}(z)$ be the element of $\mathcal{U}_{k+n}^{\prime}$ that contains $z$. Let $\tau_{n}(z)$ denote the positively oriented unit tangent vector at $z$ to $\gamma_{k, n}(z)$. Then by Lemma 9.2 and (6.3), for $m \geq n$,

$$
\left|\tau_{n}(z)-\tau_{m}(z)\right|=O\left(\sum_{l=n}^{m} \epsilon_{l}\right) .
$$

It follows from Lemma 6.4 that $\left\{\tau_{n}(z)\right\}_{n=1}^{\infty}$ is a Cauchy sequence, and $\tau_{n}(z)$ converges to some unit vector which we denote by $\tau(z)$. Then $\tau(z)$ is a unit tangent vector based at $z$ to $\Gamma_{k}$. This follows from the fact that curves $\gamma_{n, k}$ are analytic, and therefore $C^{1}$, and the fact that $\tau(z)$ is defined to be the limit of the tangent vectors $\tau_{n}(z)$. By (9.2), the functions $\tau_{n}$ converge uniformly to $\tau$, so we just need to verify that each function $\tau_{n}$ is continuous to see that $\tau$ is continuous.

Choose $z, w \in \Gamma_{k}$, and let $\gamma_{n, k}(z)$ and $\gamma_{n, k}(w)$ denote the elements of $\mathcal{U}_{n+k}^{\prime}$ that intersect $z$ and $w$, respectively. Let $L$ denote the ray based at the origin that passes through $z$. Then $L$ passes through $\gamma_{k, n}(w)$ at exactly one point, which we denote by $z^{\prime}$. We call the corresponding unit tangent vector $\tau_{n}\left(z^{\prime}\right)$. Therefore

$$
\left|\tau_{n}(z)-\tau_{n}(w)\right| \leq\left|\tau_{n}(z)-\tau_{n}\left(z^{\prime}\right)\right|+\left|\tau_{n}\left(z^{\prime}\right)-\tau_{n}(w)\right| .
$$

The distance between $z$ and $z^{\prime}$ tends to 0 as $|z-w|$ tends to 0 . So the fact that $\gamma_{k, n}(w)$ is analytic shows that $\left|\tau_{n}\left(z^{\prime}\right)-\tau_{n}(w)\right|$ can be made arbitrarily small if $|z-w|$ is sufficiently small. For the other term, the line $L$ passes through each element of 
$\mathcal{U}_{n+k}^{\prime}$ once, and the corresponding unit tangent vectors vary continuously along $L$, which shows that $\left|\tau_{n}\left(z^{\prime}\right)-\tau_{n}(z)\right|$ tends to 0 as $|z-w|$ tends to 0 as well. Putting this all together, we see that $\tau_{n}(z)$ is continuous.

Finally we show that $\Gamma_{k} \subset \mathcal{J}(f)$. For each $n, f^{n}: \Gamma_{k, n} \rightarrow A_{k+n} . \Gamma_{k, n}$ decomposes into connected components of $\left(f^{n}\right)^{-1}\left(A_{k+n} \backslash \mathbb{R}^{+}\right)$. We denote these components by $S_{n, k}$. If $z \in \Gamma_{k}$, then $z$ is contained in some set $\overline{S_{n, k}}$, and $f^{n}$ is one-to-one on the interior of $S_{n, k}$ and $f^{n}: \overline{S_{n, k}} \rightarrow A_{k+n}$ is onto. Note that $\operatorname{diam}\left(S_{n, k}\right) \rightarrow 0$ as $n \rightarrow \infty$. By definition of $\Gamma_{k}$, we have $f^{n}(z) \rightarrow \infty$ as $n \rightarrow \infty$. Let $w \in A_{k+n} \backslash \mathbb{R} \geq 0$ be a zero of $f$. Such a $w$ exists, for example, by Lemma 6.2. Then there exists an element of $f^{-n}(w)$ contained in $S_{n, k}$, and $f^{n+1}(w) \in B_{f}$, the basin of attraction containing the origin. It follows that in any neighborhood of $z,\left\{f^{n}\right\}$ cannot be an equicontinuous family.

Now we turn to a systematic labeling of the Fatou components of $f$. For $k \geq 1$, define $\Omega_{k}$ to be the Fatou component containing $B_{k-1}$. Let $D$ be the bounded complementary component of the Jordan annulus $B_{0}$. For $k \geq 0$, we may define $\Omega_{-k}$ by taking appropriate preimages, namely,

$$
\Omega_{-k}=\left\{z \in D: f^{j}(z) \in D, j=1, \ldots, k, f^{k+1}(z) \in \Omega_{1}\right\} .
$$

Note that $\Omega_{k}$ consists of exactly one connected component, since $B_{k}$ has exactly one connected component for all $k \leq 0$. By Theorem 9.3 each $\Omega_{k}$ is a distinct Fatou component for all integers $k$.

Lemma 9.4. For all $k, f\left(\Omega_{k}\right)=\Omega_{k+1}$. Each $\Omega_{k}$ is a multiply connected wandering domain.

Proof. If $k \leq 0, f\left(\Omega_{k}\right)=\Omega_{k+1}$ is true by definition. For $k \geq 1$, we know that $f\left(B_{k-1}\right) \subset B_{k} \subset \Omega_{k+1}$ by Theorem 6.12, Since $\Omega_{k+1}$ is a connected component of the Fatou set it follows that $f\left(\Omega_{k}\right) \subset \Omega_{k+1}$. Since $f$ has no asymptotic values and $\Omega_{k}$ is also a connected component of the Fatou set, we get $f\left(\Omega_{k}\right)=\Omega_{k+1}$. Each component is multiply connected since for all integers $k, B_{k} \subset \Omega_{k}$ surrounds the basin of attraction $B_{f}$ containing the origin.

Lemma 9.5. Each $\Gamma_{k}$ is a connected component of $\mathcal{J}(f)$. In fact, $\Gamma_{k}$ is simultaneously the boundary component of $\Omega_{k+1}$ which separates $\Omega_{k+1}$ from the origin and the outermost boundary component of $\Omega_{k}$.

Proof. Let $S_{n, k}$ be the sets from the proof of Theorem 9.3. By Lemma 9.2, for sufficiently large $n$, since $\Gamma_{k}$ is $C^{1}$ and an approximate circle, $\Gamma$ splits $\overline{S_{n, k}}$ into exactly two connected components. Given an $S_{n, k}$ let $S_{o}$ denote the component that is a subset of the unbounded complementary component of $\Gamma_{k}$, and we let $S_{i}$ denote the component that is a subset of the bounded complementary component $\Gamma_{k}$.

Since $f^{n}\left(\overline{S_{n, k}}\right)=A_{n+k}$, there exists a point $z \in S_{o}$ so that $\left|f^{n}(z)\right|=\frac{5}{2} R_{k+n}$. Then $f^{n+1}(z) \in B_{k+n+1}$ by Theorem 6.12. It follows that $f^{n+1}(z) \in \Omega_{k+n+2}$, and therefore $z \in \Omega_{k+1}$ by Lemma 9.4. So $\Gamma_{k}$ is a subset of the boundary of $\Omega_{k+1}$.

There also exists a point $z \in S_{i}$ so that $\left|f^{n}(z)\right|=\frac{3}{2} R_{k+n}$. Then $f^{n+1}(z) \in B_{k+n}$, so that $f^{n+1} \in \Omega_{k+n+1}$ and $z \in \Omega_{k}$. So $\Gamma_{k}$ is a subset of the boundary of $\Omega_{k}$.

Let $\Gamma$ be the connected component of the Julia set containing $\Gamma_{k}$, and suppose that $\Gamma \backslash \Gamma_{k}$ was nonempty and contained some connected component $K$. Then since $K$ is not contained in $\Gamma_{k}$, then there exists a smallest positive integer $n$ such that 
$K$ is not a subset of $\Gamma_{k, n}$. Choose a point $z \in K, z \notin \Gamma_{k, n}$, so that $f^{n}(z) \notin A_{k+n}$. Then by Lemma 6.12 we must have $z \in B_{k+n}$ or $z \in B_{k+n+1}$. This cannot happen, since $\Gamma$ is assumed to be a connected component of the Julia set.

By Lemma 8.2 and Lemma 9.4 , we know that each $\Omega_{k}$ is multiply connected. The proof of Theorem 9.3 actually shows that each $\Omega_{k}$ is infinitely connected, since the sets $\Gamma_{n, k}$ contain preimages of petals (defined in Section 6) contained in $A_{n+k}$, and these petals contain preimages of the basin of attraction containing the origin. We can decompose the complement of $\Omega_{k}$ into three types of closed regions $\Omega_{k}^{a}, \Omega_{k}^{0}$, and $\Omega_{k}^{\infty}$. $\Omega_{k}^{0}$ is the complementary component containing the origin and $\Omega_{k}^{\infty}$ is the unbounded complementary component. The remaining complementary components $\Omega_{k}^{a}$ lie between the innermost and outermost boundary components of $\Omega_{k}$. We define $\Omega_{k}^{A}$ to be the union of $\Omega_{k}$ and all the closed regions $\Omega_{k}^{a}$, so that $\Omega_{k}^{A}$ is a Jordan annulus. With this notation, $\widehat{\Omega}_{k}$ is the union of $\Omega_{k}^{0}$ and $\Omega_{k}^{A}$.

Next, for $k \geq 1$, consider a closed region $\Omega_{k}^{a}$ such that $\Omega_{k}^{a} \subset R_{k} \cdot \Omega_{n_{k}}^{p}$, for some petal $R_{k} \cdot \Omega_{n_{k}}^{p}$. The boundary of $\Omega_{k}^{a}$ is in the Julia set, and $\Omega_{k}^{a}$ contains a zero of $f$. We claim that the boundary of $\Omega_{k}^{a}$ is conformally mapped by $f$ onto the outermost boundary of $\Omega_{k}$.

Indeed, first we recall that for $\eta=R_{k}^{-3}$, the set $T_{k}^{\eta}$, defined in 8.6), gets mapped by $f$ into $B_{k}$. Therefore, we have

$$
\Omega_{k}^{a} \subset\left\{z: 4 R_{k} / 5 \leq|z| \leq 3 R_{k} / 2,\left|H_{n_{k}}\left(z / R_{k}\right)\right| \leq R_{k}^{-3}\right\} \cap R_{k} \cdot \Omega_{n_{k}}^{p} .
$$

By Lemma 8.5, some ball containing $\Omega_{k}^{a}$ gets mapped conformally onto a Jordan domain that contains $\widehat{\Omega}_{k}$. It follows that $f$ maps $\Omega_{k}^{a}$ conformally onto $\widehat{\Omega}_{k}$, so that the boundary of $\Omega_{k}^{a}$ is mapped onto the outermost boundary of $\Omega_{k}$. It further follows that inside of $\Omega_{k}^{a}$, there are conformal copies of $\Omega_{j}$ for $j \leq k$, and one conformal copy of $B_{f}$. This motivates Definition 9.6

Definition 9.6. Let $k$ be an integer. We say Fatou component $\omega$ is of $k$-type if there exists an integer $m \geq 0$ so that $f^{m}: \omega \rightarrow \Omega_{k}$ is a conformal mapping.

Such a value for $k$ is unique, since conformal mappings as we defined them are injective. Since the orbits of all points in $\Omega_{k}$ tend to $\infty$ for all $k$, the same is true for the orbits of all points in a component of $k$-type. Later, we will prove that every Fatou component that escapes is of $k$-type for some $k$. Given this universality of $\Omega_{k}$ in the Fatou set of $f$, we create Definition 9.7. Recall that $B_{f}$ is the basin of attraction for $f$ viewed as a polynomial-like mapping.

Definition 9.7. The central series of Fatou components is the union of $B_{f}$ and all components $\Omega_{k}$, where $k \in \mathbb{Z}$. The central series of Fatou components truncated at $m$ is the union of $B_{f}$ and all components $\Omega_{k}$ with $k \in \mathbb{Z}$ satisfying $k \leq m$.

As we will start to see in Section 10, the central series of Fatou components truncated at some $m$ is the primary building block for the global Fatou set of $f$. That said, there are points in the Julia set that are not in the boundary of any Fatou component. Such points are called buried points. Recall that we defined $A:=\bigcup_{k \in \mathbb{Z}} A_{k}$. Define

$$
X=\left\{z \in A: f^{n}(z) \in A \text { for all } n \geq 0\right\} .
$$

Suppose that $z \in X$. Then there exists integers $k_{n}$ so that $f^{n}(z) \in A_{k_{n}}$ for all $n \geq 0$ (we interpret $f^{0}(z)=z$ ). Then we may define the orbit sequence of $z$ to be 
$\alpha(z)=\left(k_{0}, k_{1}, k_{2}, \ldots\right)$. Notice that by Lemma 8.4, orbit sequences always satisfy the inequality $k_{j+1} \leq k_{j}+1$ for all $j \geq 0$.

We will say that a point $z \in X$ moves backwards if there exists $j \geq 0$ so that $\alpha(z)$ satisfies $k_{j+1} \leq k_{j}$. A point $z \in X$ moves backwards finitely or infinitely often if there exists finite or infinitely many values $j$ so that $\alpha(z)$ satisfies $k_{j+1} \leq k_{j}$. We will say a set $W \subset X$ moves backwards finitely often if the following two conditions hold:

(1) All $z \in W$ move backwards $m$ times for some $m \geq 1$.

(2) Let $z \in W$ and let $k_{j}$ be the $m$ th entry of $\alpha(z)$ so that $k_{j+1} \leq k_{j}$. Then if $w \in W$, then $\alpha(z)$ and $\alpha(w)$ are equal up to the $j+1$-st index.

Informally, a set moves backwards finitely often if and only if all its elements move backwards finitely often at the exact same iterates.

If $z \in A \backslash X$, then there exists a smallest integer $n$ so that $f^{n}(z) \notin A$. We may still write a finite orbit sequence for $z, \alpha(z)=\left(k_{0}, k_{1}, \ldots, k_{n-1}\right)$. Since we only require an orbit sequence to define points and sets moving backwards, we will sometimes still speak of points and sets not contained in $X$ moving backwards when the associated finite orbit sequences are defined and allow us to do so.

Lemma 9.8. Let $z \in X$ be given. If $z$ is buried then it moves backwards infinitely often.

Proof. We prove the contrapositive. Suppose that $z \in X$ moves backwards finitely often. This is true if and only if there exists integer $l \geq 0$ such that $f^{l}(z) \in X$ never moves backwards. By perhaps choosing $l$ larger, we may assume that $f^{l}(z) \in A_{k}$ for some $k \geq 1$. By Lemma 8.2, we must have $f^{l}(z) \in V_{k}$ or $f^{l}(z) \in R_{k} \cdot \mathcal{P}_{k}$. If $f^{l}(z) \in R_{k} \cdot \mathcal{P}_{k}$, then from the discussion following Lemma 8.2 we must have

$$
f^{l}(z) \in\left\{z: 4 R_{k} / 5 \leq|z| \leq 3 R_{k} / 2,\left|H_{n_{k}}\left(z / R_{k}\right)\right| \leq R_{k}^{-3}\right\} \subset\left\{z:|z| \leq 3 R_{k} / 2\right\} .
$$

By Lemma 6.12 the inner boundary component of $V_{k}, C\left(0,3 R_{k} / 2\right)$, gets mapped inside of $B_{k}$. Therefore, by the maximum principle for holomorphic functions, $f^{l+1}(z) \in\left\{z:|z|<R_{k+1} / 4\right\}$. Therefore, if we let $\alpha\left(f^{l}(z)\right)=\left(k_{0}, k_{1}, \ldots\right)$ denote the orbit sequence of $f^{l}(z)$, we must have $k_{1} \leq k_{0}$. But this contradicts the fact that we assumed that $f^{l}(z)$ never moves backwards. Therefore, we must have $f^{l}(z) \in V_{k}$. By similar reasoning, we conclude that for all $j \geq 1, f^{l+j}(z) \in V_{k+j}$, and it follows that $f^{l}(z) \in \Gamma_{k}$, the outermost boundary of $\Omega_{k}$. It follows that $z$ is on the boundary of some Fatou component for $f$.

We will see later that $z$ is buried if and only if $z$ moves backwards infinitely often. For the rest of the paper, we will refer to the points that move backwards infinitely often as $Y$.

\section{A DETAILED DESCRIPTION OF THE DYNAMiCs OF $f$}

We can now offer a complete description of the Fatou and Julia set, along with several other dynamical consequences. These will follow Theorem 10.1, which we will prove in Sections 11 13, For convenience, we will often refer to a sum of diameters of the form below as an $\left(s+\epsilon_{0}\right)$-sum. In this section and future sections, we will let $s=\operatorname{dim}_{H}\left(\partial B_{f}\right)$, where $B_{f}$ is the basin of attraction of $f$ containing the origin. 
Theorem 10.1. Let $\epsilon_{0}>0$ be such that $s+\epsilon_{0}<2$, and $k \in \mathbb{Z}$ be given. Then $R$ may be chosen large enough so that

$$
\sum_{\omega \subset \Omega_{k}^{A}} \operatorname{diam}(\omega)^{s+\epsilon_{0}}<\infty
$$

where the sum is taken over every Fatou component $\omega \subset \Omega_{k}^{A}$ which is of $j$-type for some $j \geq 1$.

We would like to emphasize that we are summing over all Fatou components contained inside of $\Omega_{k}^{A}$ of $j$-type for any $j \geq 1$, not just one fixed $j$. This theorem cannot be significantly strengthened to include all Fatou components of $j$-type for $j \in \mathbb{Z}$. Indeed, we will see that if $B_{f}^{\prime}$ is a connected component of $\left(f^{m}\right)^{-1}\left(B_{f}\right)$ for some $m \geq 1$ contained inside of $\Omega_{k}^{A}$, there exists infinitely many Fatou components surrounding $B_{f}^{\prime}$ with diameter larger than the diameter of $B_{f}^{\prime}$. Compare to (20.1) in Bis18. n. 455.

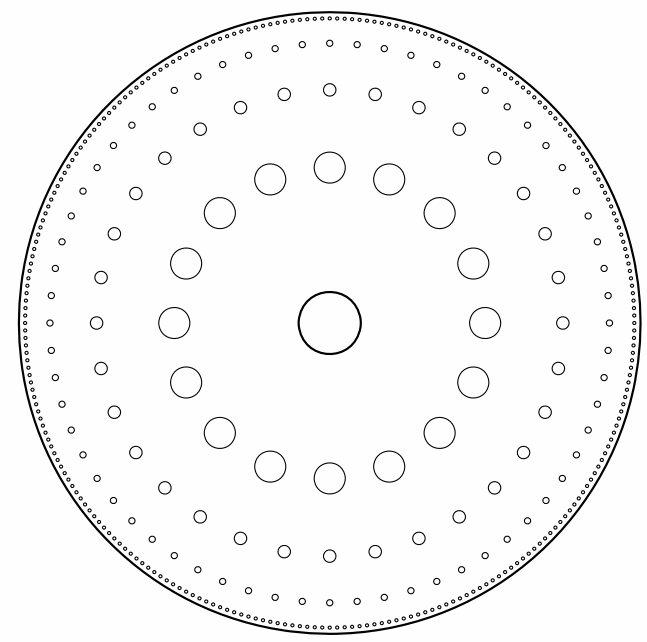

Figure 7. A schematic for a Fatou component $\Omega_{k}, k \geq 1$. Every hole in $\Omega_{k}^{A}$ is bounded by the outermost boundary of a Fatou component of $j$-type, $j \geq k$. This forms the first layer of holes, which we see pictured above. Every hole contains a copy of the central series of Fatou components truncated at some $j \geq k$; the holes in all of these copies induce the second layer of holes in $\Omega_{k}^{A}$. This introduces a third layer of holes, and the process continues inductively. The set $Y$ of points that move backwards infinitely often coincides with the set of all points that are contained inside of a hole in every layer.

Let's first geometrically interpret what this sum means. Figure 7 shows a schematic diagram for the Fatou component $\Omega_{k}, k \geq 1$. As discussed in Section [9] $\Omega_{k}$ is infinitely connected. The 'holes' in Figure 7 correspond to the components $\Omega_{k}^{a}$. The innermost ring of holes is contained inside of the collection of petals $R_{k} \cdot \mathcal{P}_{n_{k}}$, defined in Section 8 . There is exactly one hole for each individual petal $R_{k} \cdot \Omega_{n_{k}}^{p}$, and $f$ maps the outermost boundary of each of these holes conformally 
onto the outermost boundary component of $\Omega_{k}$ with small distortion by Lemma 8.5. Therefore, each hole contained in a petal contains a preimage or copy of the central series of Fatou components truncated at $k$, and Lemma 8.5 says that this preimage is almost an affine rescaling of the central series truncated at $k$.

When $k \geq 1$, the other rings of holes of $\Omega_{k}$ are mapped to rings of holes in $\Omega_{k+1}$. The second ring of holes maps into $R_{k+1} \cdot \mathcal{P}_{n_{k+1}}$, and in general, each ring of holes moves one ring inward under iteration by $f$. Therefore, each element of the other rings of holes in Figure 7 is eventually mapped conformally into $R_{j} \cdot \mathcal{P}_{n_{j}}$ for some $j>k$, and therefore the same reasoning shows that those holes contain a preimage or copy of the central series of Fatou components truncated at $j$.

The discussion above also applies to $\Omega_{k}$ for $k \leq 0$, with some minor adjustments. In this case, recall that $\Omega_{k}$ is defined to be the connected component of $\left(f^{1-k}\right)^{-1}\left(\Omega_{1}\right)$ that surrounds the origin. Each complementary component $\Omega_{k}^{a}$ gets mapped conformally by $f^{1-k}$ onto some component $\Omega_{1}^{a}$, so it also contains a preimage of the central series of Fatou components truncated at some $j \geq 1$. From this we deduce that the boundary of every complementary component of $\Omega_{k}$ contained in $\Omega_{k}^{A}$ is the outermost boundary of some Fatou component of $j$-type, where $j \geq 1$.

To summarize the above discussion using the informal language of holes, we have replaced all of the holes in $\Omega_{k}$ by appropriate truncated copies of the central series of Fatou components. These truncated copies introduce a new second layer of holes contained in $\Omega_{k}$, introduced by the corresponding copies of the truncated central series of Fatou components. The reasoning above shows that every hole in this second layer contains a truncated copy of the central series of Fatou components, which introduces a third layer of holes in $\Omega_{k}$, and so on.

From this discussion we see that the truncated copies of the central series of Fatou components serve as the main building block for the Fatou and Julia set of $f$. We fill all the holes in the central series by the appropriate truncated copies of the central series, and repeat this procedure for all the new, smaller holes that appear. Theorem 10.1 can now be interpreted as saying that the $\left(s+\epsilon_{0}\right)$-sum of diameters of every hole in every layer of holes contained in $\Omega_{k}^{A}$ is finite (in fact, it is slightly stronger, since many Fatou components may be contained inside of the same hole).

We can also see from this discussion that every hole in the $m$ th layer described above moves backwards $m$ many times. Therefore, we see that the set $Y$ of points that move backwards infinitely often coincides with the set of points that are contained inside a hole for every layer of holes in $\Omega_{k}^{A}$. For each $m$, the $m$ th layer of holes is a covering of $Y \cap \Omega_{k}^{A}$, so since the sum in Theorem 10.1 converges we will be able to deduce the following consequence.

Corollary 10.2. Let $\epsilon_{0}>0$ be given, and suppose that $R$ is chosen so that (10.1) converges. Then for all $k$, $\operatorname{dim}_{\mathrm{H}}\left(Y \cap \Omega_{k}^{A}\right) \leq s+\epsilon_{0}$, so that $\operatorname{dim}_{\mathrm{H}}(Y) \leq s+\epsilon_{0}<2$.

For the remainder of this section we will describe the dynamical consequences of Theorem 10.1 and Corollary 10.2

Theorem 10.3. Let $\mathcal{C}$ be defined as in (8.1). Suppose that $\omega$ is a Fatou component of $f$ and $\omega$ is not contained in $\mathcal{C}$. Then there exists a unique integer $k \in \mathbb{Z}$ such that $\omega$ is of $k$-type.

Proof. Let $\omega$ be a Fatou component of $f$ which is not a subset of $\mathcal{C}$. First, observe that for any integer $k$, we must have $B_{k} \cap \omega=\emptyset$ or $B_{k} \cap \omega=\Omega_{k}$ since $B_{k}$ is a subset 
of the Fatou component $\Omega_{k}$. In the latter case, the $\omega=\Omega_{k}$, which is of $k$-type. Therefore, $\omega \subset A:=\bigcup_{k \in \mathbb{Z}} A_{k}$.

Choose $j$ so that $\omega \subset A_{j}$. If $j \leq 0$, then $f^{1-j}(\omega) \subset A_{1}$, so we may assume without loss of generality that $j \geq 1$. Since $\partial \omega \subset \mathcal{J}(f)$, then by Lemma 8.2, we either have $\omega \subset R_{j} \cdot \Omega_{n_{j}}^{p}$ or we have $\omega \subset V_{j}$. If $\omega \subset R_{j} \cdot \Omega_{n_{j}}^{p}$, then $\omega$ moves backwards once. If $\omega \subset V_{j}$, then there is an integer $l \geq 1$ so that $f^{l}(\omega) \subset R_{j+l} \cdot \Omega_{n_{j+l}}^{p}$. Otherwise, by (9.1), $\omega \subset \Gamma_{j, l}$ for all $l \geq 1$, which would imply that $\omega$ is a subset of the outermost boundary of $\Omega_{j}$, which is impossible since $\omega$ is open.

Therefore, there exists $l \geq 0$ so that $f^{l}(\omega) \subset R_{j+l} \cdot \Omega_{n_{j+l}}^{p}$. Then for any $k \in \mathbb{Z}$, we either have $f^{l+1}(\omega) \cap B_{k}=\Omega_{k}$ or $f^{l+1}(\omega) \cap B_{k}=\emptyset$. If there exists a $k$ such that $f^{l+1}(\omega) \cap B_{k}=\Omega_{k}$, then $\omega$ is of $k$-type by Lemma 8.5. Otherwise, $f^{l+1}(\omega) \subset A$.

It follows from the reasoning above that if $\omega$ is not of $k$-type for some integer $k$, then $\omega \subset X$, where $X$ is the set of points defined in (9.3) that remain in $A$ under all iterates of $f$. In fact, the reasoning above shows that $\omega$ would have to move backwards infinitely often, which would imply that $\omega \subset Y$, which is impossible by Corollary 10.2

Corollary 10.4. We have the following:

(1) $\mathcal{J}(f) \backslash \mathcal{C} \subset X$.

(2) For each $z \in \mathcal{J}(f) \cap X$, the point $z$ is buried if and only if $z \in Y$.

Proof. To verify the first statement, suppose that $z \in \mathcal{J}(f) \backslash \mathcal{C}$. Every $z \in \mathbb{C}$ belongs to exactly one of $K_{f}$, int $B_{k}$ for some $k \in \mathbb{Z}$, or $A_{k}$ for some $k \in \mathbb{Z}$. If there exists an $l \geq 0$ so that $f^{l}(z) \in K_{f}$, then $z \in \mathcal{C}$ by definition. If there exists an $l \geq 0$ such that $f^{l}(z) \in \operatorname{int} B_{k}$ for some $k \in \mathbb{Z}$, then $z$ belongs to the Fatou set of $f$ by Corollary 6.13. Thus if $z \in \mathcal{J}(f) \backslash \mathcal{C}$, for each $l \geq 0$ we have $f^{l}(z) \in A_{k}$ for some integer $k$. Therefore, $z \in X$.

To verify the second claim, recall that we have shown that if $z \in X$ is buried then it moves backwards infinitely often. If $z$ is not buried, then by definition it is the boundary of some Fatou component $\omega$. If $\omega \subset \mathcal{C}$, then $z \notin X$. So $\omega$ is not contained in $\mathcal{C}$, and by Theorem 10.3 , there exists $m \geq 0$ and $k \in \mathbb{Z}$ so that $f^{m}$ is conformal on $\omega$ and $f^{m}(\omega)=\Omega_{k}$. Since $f\left(\Omega_{k}\right)=\Omega_{k+1}, z$ moves backwards only finitely often.

We have shown that if a Fatou component is not a subset of $\mathcal{C}$, then that component belongs to the escaping set of $f$. Recall that for an entire function $f$, we define the escaping set as

$$
I(f)=\left\{z:\left|f^{n}(z)\right| \rightarrow \infty\right\} .
$$

Choose some number $S_{0}$ so that there exists $z$ with $|z|=S_{0}$ so that $z \in I(f)$ (for example, choose $S_{0}$ so that $|z|=S_{0} \subset B_{1}$ ). Then define inductively

$$
S_{n+1}=\max _{|z|=S_{n}}|f(z)| \text {. }
$$

We define the fast escaping set as

$$
A(f)=\left\{z: \text { there exists } k \geq 0 \text { so that }\left|f^{n+k}(z)\right| \geq S_{n} \text { for all } n \geq 0\right\} .
$$

The fast escaping set does not depend on the initial choice of $S_{0}$, as long as $|z|=S_{0}$ contains a point $z \in I(f)$. For more on the fast escaping set, we refer the reader to RS12. 
Lemma 10.5. Let $\mathcal{C}$ be defined as in (8.1). Suppose that $\omega$ is a Fatou component of $f$ and $\omega$ is not contained in $\mathcal{C}$. Then $\omega \subset A(f)$.

Proof. We begin by defining $\left\{S_{n}\right\}_{n=0}^{\infty}$. Let $S_{0}=\frac{1}{4} R_{2}$. Such a choice of $S_{0}$ is valid by Theorem 6.12 , for if $|z|=S_{0}$, then $z$ is on the outer boundary of $B_{1}$ and therefore in the escaping set. It also follows from Theorem 6.12 that for all $n \geq 0$ we have the inequality

$$
S_{n} \leq \frac{1}{4} R_{n+2}
$$

For any integer $k \geq 3$, we first claim that the Fatou component $\Omega_{k} \subset A(f)$. For such $k$, observe that if $z \in \Omega_{k}$, then $|z| \geq R_{k-1} / 4$. Indeed, the Fatou component $\Omega_{k-1}$ contains the annulus $B_{k-2}$, and $|z|=R_{k-1} / 4$ is the outer boundary of $B_{k-2}$. Therefore, if $z \in \Omega_{k}$ for $k \geq 3$, then $|z|=\left|f^{0}(z)\right| \geq S_{0}$. Since $f^{n}(z) \in \Omega_{k+n}$ for $n \geq 1$ and $k \geq 3$, we have $\left|f^{n}(z)\right| \geq R_{k+n-1} / 4 \geq R_{n+2} / 4 \geq S_{n}$. Therefore, $\Omega_{k} \subset A(f)$.

By Theorem 2.2 of [RS12], $A(f)$ is completely invariant under $f$. Therefore, if $k \leq 2$, we have $\Omega_{k} \subset A(f)$ as well. Similarly, if $\omega$ is a Fatou component which is not a connceted component of $\mathcal{C}, \omega$ is of $k$-type for some integer $k$, and belongs to $A(f)$ as well.

We will sometimes call Fatou components in $A(f)$ escaping Fatou components. Next, we define the bungee set as

$B U(f)=\left\{z:\right.$ there exists $n_{k}$ and $n_{j}$ so that $\left|f^{n_{k}}(z)\right| \rightarrow \infty$ and $f^{n_{j}}(z)$ is bounded $\}$.

We define the bounded orbit set as

$$
B O(f)=\left\{z: f^{n}(z) \text { is bounded }\right\} .
$$

Every point $z \in \mathbb{C}$ is contained in exactly one of $I(f), B U(f)$, or $B O(f)$. All of the elements of $B U(f)$ belong to $Y$, and every point in $B O(f)$ that does not eventually map into $K_{f}$, the filled Julia set of the polynomial-like mapping $f$, also belongs to $Y$. $Y$ also contains points in $I(f)$. However, since these points move backwards infinitely often, they cannot belong to $A(f)$ for our function $f$.

Corollary 10.6. The sets $I(f) \backslash A(f), B U(f)$, and $B O(f) \backslash \mathcal{C}$ all have Hausdorff dimension $\leq s+\epsilon_{0}<2$.

Proof. $B U(f) \subset Y$ and $B O(f) \backslash \mathcal{C} \subset Y$, and $Y$ has Hausdorff dimension $\leq s+\epsilon_{0}$ by Corollary 10.2. If $z \in I(f) \backslash A(f)$, then $z \notin \mathcal{C}$, nor can we have $z$ inside an escaping Fatou component or the boundary of a Fatou component of $k$-type for any integer $k$, since these sets belong to $A(f)$. Therefore $z$ must be in $Y$, and $I(f) \backslash A(f) \subset Y$, and the result follows.

We now know that Fatou components are either inverse images of the basin of attraction $B_{f}$ of $f$ viewed as a polynomial-like mapping, or are of $k$-type for some integer $k$.

Definition 10.7. Let $\omega$ be a Fatou component of $f$.

(1) If $\omega \subset \mathcal{C}$ is a Fatou component, then there is an integer $m \geq 1$ such that $f^{m}(\omega)=B_{f}$. In this case, we call $\omega$ a basin or basin component.

(2) If $\omega$ is $k$-type for $k \geq 1$, we call $\omega$ round.

(3) If $\omega$ is $k$-type for $k \leq 0$ we call $\omega$ wiggly. 
The reason for the naming convention above is as follows. As $k \rightarrow-\infty$, the innermost and outermost boundaries of components $\left\{\Omega_{k}\right\}_{k \leq 0}$ 'wiggle' and approximate the fractal boundary of the basin of attraction $B_{f}$. Round components are far enough away from the fractal boundary to still have approximately circular innermost and outermost boundaries.

Recall the sequence $\left\{\epsilon_{k}\right\}_{k=1}^{\infty}$ defined in (6.3). In Section 9 we showed that the $\epsilon_{k}$ 's determined the deviation from round circles of the innermost and outermost boundary components of $\Omega_{k}, k \geq 1$. For any $\delta>0$, we could define $f$ so that $\sum_{k=1}^{\infty} \epsilon_{k}<\delta$.

Corollary 10.8. Let $\left\{\epsilon_{k}\right\}_{k=1}^{\infty}$ be defined as in (6.3), and suppose $\sum_{k=1}^{\infty} \epsilon_{k}=\delta$ for some $\delta>0$. The boundary of any escaping Fatou component is the union of $C^{1}$ curves. If the Fatou component is round, the all of the boundary components are $O(\delta)$-approximate circles.

Proof. All escaping components are of $k$-type for some $k$, so it suffices to show this for $\Omega_{k}$ by Lemma 8.5 and Lemma 9.1 . Since all the boundary components of $\Omega_{k}$ are escaping by Corollary 10.4, they are the boundaries of Fatou components of $j$-type for $j \geq 1$. By Lemma 8.5. Fatou components of $j$-type map conformally onto $\Omega_{j}$ and their boundaries are also $C^{1}$. By Lemma 9.1, they are $O(\delta)$-approximate circles when $k \geq 1$.

We can now offer a full description of the Julia set.

Theorem 10.9. The Julia set can be decomposed into three sets:

(1) The set $Y$ of points which move backwards infinitely often.

(2) The $C^{1}$ components that escape to $\infty$. These components are always the outermost boundary component of some Fatou component of $k$-type.

(3) Inverse images of $J_{f}$, the Julia set of the polynomial-like map $f$.

Corollary 10.10. $J(f)$ has zero Lebesgue measure.

Proof. The Julia set is the disjoint union of the the set of points that move backwards infinitely often which has Hausdorff dimension less than 2 by Corollary 10.2 . countably many $C^{1}$ curves, and countably many quasicircles with Hausdorff dimension strictly less than 2 .

We conclude the section by recording a lemma describing the nice geometry of the round Fatou components $\Omega_{k}, k \geq 1$; we refer the reader again to Figure 7 . The proof follows from some basic calculations using the fact that $f$ looks like a power mapping on some portions of $\Omega_{k}$, along with using Lemma 8.5. A similar discussion is found in Section 19 of [Bis18, and we omit the details.

Lemma 10.11 (The shape of round Fatou components). Choose some Fatou component $\Omega_{k}$ for some fixed $k \geq 1$. Define $d_{j}=2\left(n_{k}+\cdots+n_{j-1}\right)$ for $j>k$. Then $\Omega_{k}$ has the following geometric properties

(1) For all $j \geq k$, there are $n_{j} \cdot 2^{d_{j}}$ many boundary components of $\Omega_{k}$ which lie distance approximately $R_{k} \cdot 2^{-d_{j}}$ from the outermost boundary component of $\Omega_{k}$. We call these components the jth ring of $\Omega_{k}$.

(2) The boundary components in the $j$ th ring of $\Omega_{k}$ are approximately distance $R_{k} n_{j}^{-1} 2^{-d_{j}}$ apart from each other and lie on a $O(\delta)$-approximately round circle.

(3) All boundary components of $\Omega_{k}$ arise in this manner for some $j \geq k$. 


\section{The Proof of Theorem 10.1] A labeling system for holes}

In this section, we formally construct a sequence of coverings $\mathcal{C}_{m}$ of $Y \cap A_{1}$ which correspond to the $m$ th layer of holes in $\Omega_{k}^{A}$ described in the previous section. We will focus on the case in Theorem 10.1 with $k=1$. It is straightforward to modify our techniques to other integers $k$.

Our initial covering $\mathcal{C}_{0}$ will have exactly one element, $A_{1}$, the annulus defined in Section 6. Notice that by the proof of Theorem 9.3. $A_{1}$ contains the outermost boundary component of $\Omega_{1}$, so that $\widehat{A}_{1}$ contains $\widehat{\Omega}_{1}$. $A_{1}$ and $\Omega_{1}$ both have diameter comparable to $R_{1}$. We first describe how to construct $\mathcal{C}_{1}$ from $\mathcal{C}_{0}$. For each $z \in$ $A_{1} \cap Y$, by definition, there is a smallest positive integer $n$ so that $f^{n}(z) \in A_{k}$ for $k \leq n$. It is possible that $k \leq n$ is a negative integer. Therefore $z$ belongs to one connected component of $f^{-n}\left(A_{k}\right)$, a Jordan annulus which is a proper subset of $A_{1}$. We denote such a component by $W_{k}^{n}$. The collection of all distinct components obtained by doing this procedure for all $z \in Y$ is denoted by $\mathcal{C}_{1}$.

Before proceeding further, we would like to remark on some potentially confusing notation. The convention of referring to elements in $\mathcal{C}_{1}$ as $W_{k}^{n}$ is ambiguous. Indeed, there are $2^{N}$ many elements of $\mathcal{C}_{1}$ that could be called $W_{1}^{1}$, one for each petal $R_{1} \cdot \Omega_{n_{1}}^{p}$. With this ambiguity in mind, we will adopt the convention that the notation $W_{k}^{n} \in \mathcal{C}_{1}$ always refers to a single element. We will say that an element $X \in \mathcal{C}_{1}$ is of the form $W_{k}^{n}$ if it could be denoted by $W_{k}^{n}$ using the procedure above for some $z \in X$. Therefore every element of $\mathcal{C}_{1}$ is of the form $W_{k}^{n}$ for some positive integer $n$ and some integer $k \leq n$. This slight abuse of notation will not be an issue in what follows; we will exclusively refer to either single elements $W_{k}^{n}$ or the collection of all elements of the form $W_{k}^{n}$. We will make it clear in each context if we are referring to a single element versus a collection of elements.

With this in mind we now describe how to obtain $\mathcal{C}_{2}$ from $\mathcal{C}_{1}$. Let $W_{k}^{n} \in \mathcal{C}_{1}$ be given, and choose $z \in Y \cap W_{k}^{n}$. Then $f^{n}(z) \in A_{k}$ for $k \leq n$. But since $z \in Y$, there exists a smallest $q$ so that $f^{n+q}(z) \in A_{j}$ for $j \leq k+q-1$. $z$ is contained in a component of $\left(f^{n+q}\right)^{-1}\left(A_{j}\right) \subset A_{1}$, which we denote by $W_{j}^{n+q}$. Therefore, for all $z \in Y \cap W_{k}^{n}$ there is a Jordan annulus $W_{j}^{n+q} \subset W_{k}^{n}$ containing $z$ which moves backwards twice. We define $\mathcal{C}_{2}$ to be the collection of all distinct Jordan annuli obtained by applying this procedure to each element of $\mathcal{C}_{1}$.

We proceed inductively to construct $\mathcal{C}_{m+1}$ from $\mathcal{C}_{m}$. Let $z \in W_{k_{m}}^{n_{m}} \cap Y$ for some element $W_{k_{m}}^{n_{m}} \in \mathcal{C}_{m}$. Then since $z \in Y$, there exists a smallest $q$ so that $f^{n_{m}+q}(z) \in$ $A_{j}$ for $j \leq k_{m}+q-1$. Therefore $z$ is an element of some connected component of $\left(f^{n_{m}+q}\right)^{-1}\left(A_{j}\right)$, and we denote this component by $W_{j}^{n_{m}+q}$. Therefore, for all $z \in Y \cap W_{k_{m}}^{n_{m}}$, there is a Jordan annulus $W_{j}^{n_{m}+q} \subset W_{k_{m}}^{n_{m}}$ which moves backwards $m+1$ many times. The collection $\mathcal{C}_{m+1}$ is the collection of all distinct components obtained by applying this procedure to each element of $\mathcal{C}_{m}$.

We summarize several properties of the coverings $\mathcal{C}_{m}$ in Lemma 11.1

Lemma 11.1. Let $m \geq 0$. The collection of sets $\mathcal{C}_{m}$, has the following properties.

(1) $\mathcal{C}_{m}$ is a countable union of Jordan annuli which cover $Y \cap \Omega_{1}^{A}$.

(2) $\mathcal{C}_{m+1}$ is a refinement of $\mathcal{C}_{m}$, i.e, every element in $\mathcal{C}_{m+1}$ is a subset of an element in $\mathcal{C}_{m}$.

(3) Let $W_{k}^{n} \in \mathcal{C}_{m}$. Then $W_{k}^{n}$ moves backwards $m$ times. 
(4) If $W_{k}^{n} \in \mathcal{C}_{m}$, then $W_{k}^{n}$ contains the outermost boundary component of exactly one Fatou component of $k$-type that moves backwards $m$ times, but not $m+1$ times.

(5) Suppose $\omega$ is a Fatou component of $k$-type contained in $\Omega_{1}^{A}$. Then there is a positive integer $m$ so that $\omega$ moves backwards $m$ times, but $\omega$ does not move backwards $m+1$ times. Moreover, there exists a unique element $W_{k}^{n} \in \mathcal{C}_{m}$ so that $W_{k}^{n}$ contains the outermost boundary of $\omega$.

Proof. We only need to discuss (4) and (5). For (4), if $W_{k}^{n} \in \mathcal{C}_{m}$, then by definition $f^{n}\left(W_{k}^{n}\right)=A_{k}$ and $W_{k}^{n}$ moves backwards $m$ many times. $A_{k}$ contains the outermost boundary of $\Omega_{k}$, so $W_{k}^{n}$ must contain a Fatou component of $k$-type that moves backwards $m$ times. Since $f\left(\Omega_{k}\right)=\Omega_{k+1}$, this component does not move backwards again, which gives uniqueness, since all other Fatou components whose outermost boundary is contained in $W_{k}^{n}$ will move backwards more than $m$ many times.

For (5), the existence of such an $m$ is guaranteed by the definition of $k$-type and the fact that $f\left(\Omega_{k}\right)=\Omega_{k+1}$. Therefore, the outermost boundary component of $\omega$ moves backwards $m$ times. Suppose $l \geq 0$ is such that $f^{l}(\omega)=\Omega_{k}$ and $f^{l}$ is conformal on $\omega$. The existence of $W_{k}^{n}$ follows from the fact that outermost boundary of $f^{l}(\omega)$ is contained in $A_{k}$, so the outermost boundary of $\omega$ will be contained in some element $W_{k}^{n}$ in $\mathcal{C}_{m}$.

We move on to describing how to change the covering $\mathcal{C}_{m}$ by topological annuli into a simpler covering $\widehat{\mathcal{C}}_{m}$ by topological balls. The elements of $\widehat{\mathcal{C}}_{m}$ are going to be topological disks that cover the holes described in Sections 9 and 10, Let $W_{k}^{n} \in \mathcal{C}_{1}$ be given. Then $\widehat{W}_{k}^{n}$ is a topological disk with the same diameter as $W_{k}^{n}$. $\widehat{W}_{k}^{n}$ is contained inside of exactly one element $\widehat{W}_{n}^{n}$, with $W_{n}^{n} \in \mathcal{C}_{1}$. Indeed, suppose $W_{k}^{n} \in \mathcal{C}_{1}$. Then $f^{n-1}\left(W_{k}^{n}\right)$ is a subset of some petal contained in $\mathcal{P}_{n}$. From the discussion of the proof of Lemma 8.2 , we know that

$$
f^{n-1}\left(W_{k}^{n}\right) \subset\left\{z: 4 R_{n} / 5 \leq|z| \leq 3 R_{n} / 2, H_{n_{n}}\left(z / R_{n}\right) \leq R_{k}^{-3}\right\}=S .
$$

(We clarify that $n_{n}=2^{N+n-1}$ ). We also know that $f$ conformally maps $S$ onto some topological disk whose boundary is contained in $B_{n}$, so that $S$ contains a connected component of $f^{-1}\left(A_{n}\right)$. It follows that $\widehat{W}_{k}^{n}$ is contained inside of exactly one element $\widehat{W}_{n}^{n}$, with $W_{n}^{n} \in \mathcal{C}_{1}$.

We define $\widehat{\mathcal{C}}_{1}$ to be the collection of all topological disks of the form $\widehat{W}_{n}^{n}, n \geq$ 1. Observe that Theorem 6.12 and Lemma 8.2 imply that $\widehat{\mathcal{C}}_{1}$ has the following maximality property: If $\widehat{W}_{n}^{n} \in \widehat{\mathcal{C}}_{1}$, there is no $W_{k}^{n} \in \mathcal{C}_{1}$ so that $\widehat{W}_{n}^{n}$ is a proper subset of $\widehat{W}_{k}^{n}$. This implies that $\widehat{\mathcal{C}}_{1}$ covers all the points that $\mathcal{C}_{1}$ does. We will continue to use the convention that $\widehat{W}_{k}^{n}$ refers to a single element, and refer to the collection of all elements that could be labeled the same way as the elements of the form $\widehat{W}_{k}^{n}$.

To inductively obtain $\widehat{\mathcal{C}_{m+1}}$, assume that $\widehat{\mathcal{C}_{m}}$ has been constructed and satisfies the maximality property that no element $\widehat{W}_{k}^{n} \in \widehat{\mathcal{C}}_{m}$ is a proper subset of $\widehat{W}_{j}^{n}$ where $W_{j}^{n} \in \mathcal{C}_{m}$. Start with take $\widehat{W}_{k}^{n} \in \widehat{\mathcal{C}}_{m}$. Then $k \geq 1$; otherwise $\widehat{W}_{k}^{n}$ is a proper subset of $\widehat{W}_{k+1}^{n}$ for some $W_{k+1}^{n} \in \mathcal{C}_{m}$. Observe that $\widehat{W}_{k}^{n}$ contains a sequence of components $W_{j}^{n} \in \mathcal{C}_{m}$ for $j \leq k$. Fix $W_{j}^{n}$, and consider the elements of $\mathcal{C}_{m+1}$ contained inside of $W_{j}^{n}$ of the form $W_{j+q-1}^{n+q}$. If $j \geq 1$, then all $q \geq 1$ occur. If $j \leq 0$, then $q$ must 
satisfy $q \geq 2-j$. Either way, for each valid choice of $q$, the polynomial hulls of the components of the form $W_{k+q-1}^{n+q}$ determine the elements of $\widehat{\mathcal{C}}_{m+1}$ inside of $W_{j}^{n}$. Doing this for all $j \leq k$, we obtain all of the elements of $\widehat{\mathcal{C}}_{m+1}$ contained in $\widehat{W}_{k}^{n}$. $\widehat{\mathcal{C}}_{m+1}$ is the collection of all elements obtained in this way for each $\widehat{W}_{k}^{n} \in \mathcal{C}_{m}$.

We summarize the properties of the coverings $\widehat{\mathcal{C}_{m}}$ below.

Lemma 11.2. The collection of sets $\widehat{\mathcal{C}}_{m}, m \geq 0$, has the following properties.

(1) $\widehat{\mathcal{C}}_{m}$ is a countable collection of topological disks which cover $Y \cap \Omega_{1}^{A}$.

(2) $\widehat{\mathcal{C}}_{m+1}$ is a refinement of $\widehat{\mathcal{C}}_{m}$.

(3) Let $\widehat{W}_{k}^{n} \in \widehat{\mathcal{C}}_{m}$. Then $\widehat{W}_{k}^{n}$ moves backwards m many times.

(4) If $\widehat{W}_{k}^{n} \in \widehat{\mathcal{C}}_{m}$. Then $k \geq 1$, and $\widehat{W}_{k}^{n}$ contains the outermost boundary of exactly one Fatou component of $k$-type that moves backwards $m$ many times, but not $m+1$ many times.

(5) Maximality: if $\widehat{W}_{k}^{n} \in \widehat{\mathcal{C}}_{m}$, then there does not exist a different element $W_{j}^{n} \in \mathcal{C}_{m}$ so that $\widehat{W}_{k}^{n}$ is a proper subset of $\widehat{W}_{j}^{n}$.

Proof. The reasoning for (4) follows similarly as it does in Lemma 11.1. The only thing left to be checked is maximality. But this follows from the fact that inside of each $W_{j}^{n} \subset \widehat{W}_{k}^{n}$ in the construction above, we refined by using components of the form $W_{j+q-1}^{n+q}$.

Example 11.3. We would like to illustrate what happens when we refine $\widehat{W}_{n}^{n} \in \widehat{\mathcal{C}}_{1}$ into components that belong to $\widehat{\mathcal{C}_{2}}$. This example is actually quite universal and will motivate the technical lemmas in the next two sections. The idea behind this construction, pulling back a construction on the central series of Fatou components to all other Fatou components of $k$-type, will be a important theme in the following sections.

When $k \geq 1$, we may refine $A_{k} \cap Y$ similarly to how we constructed $\mathcal{C}_{1}$ and $\widehat{\mathcal{C}_{1}}$. We call this new collection of topological disks $\mathcal{V}_{k}$, and we denote individual elements of $\mathcal{V}_{k}$ by $V_{k}^{n}$, where $n$ is the largest integer so that $V_{k}^{n}$ contains a copy of the central series of Fatou components truncated at $n$ which moves backwards exactly once. Similar to our conventions above, we denote a single element of $\mathcal{V}_{k}$ as $V_{k}^{n}$ despite the ambiguity of the notation, and refer to a collection of elements of $\mathcal{V}_{k}$ as being of the form $V_{k}^{n}$. Figure 8 gives a schematic illustration of $\mathcal{V}_{k}$ 's for some $k \geq 1$.

When $k \leq 0$ and $z \in A_{k} \cap Y$, then $f^{1-k}(z) \in A_{1} \cap Y$, and therefore $z \in \widehat{W}_{n}^{n} \subset A_{1}$ for some $\widehat{W}_{n}^{n} \in \widehat{\mathcal{C}}_{1}$. In this case, define $V_{k}^{n}$ to be the component of $\left(f^{1-k}\right)^{-1}\left(\widehat{W}_{n}^{n}\right)$ that contains $z$. Doing this for all $z$, we obtain a countable collection of topological disks which we denote by $\mathcal{V}_{k}$. We make the same notational conventions as we do when $k \geq 1$; individual elements are referred to as $V_{k}^{n}$ and a collection of components is of the form $V_{k}^{n}$ if each individual component could be labeled as $V_{k}^{n}$. Figure 9 gives a schematic illustration of $\mathcal{V}_{k}$ 's for some $k$ much smaller than 0.

Let's turn our attention back to $\widehat{W}_{n}^{n} \in \widehat{\mathcal{C}}_{1}, \widehat{W}_{n}^{n} \subset \Omega_{1}^{A} . \widehat{W}_{n}^{n}$ corresponds to one complementary component of $\Omega_{1}$ in $\Omega_{1}^{A}$ that contains a central series of Fatou components truncated at $n$. Let $\omega_{j}, j \leq n$ denote the Fatou components of $j$-type that make up this copy of the central series. The $n$th iterate of $f$ conformally maps this copy of the central series inside $\widehat{W}_{n}^{n}$ to the actual central series of Fatou 
components truncated at $n$. Therefore elements of $\widehat{\mathcal{C}}_{2}$ contained inside of $\widehat{W}_{n}^{n}$ are precisely the preimages of the elements of $\mathcal{V}_{j}$ for $j \leq n$.

\section{The $s$-SUm of the FATOU COMPonents: REFINING AWAY FROM $B_{f}$}

In the next two sections, we show that our refining and polynomial hull taking refinements to the coverings in the previous section result in a decreased $\left(s+\epsilon_{0}\right)$ sum of the diameters of the elements in the coverings. First we need an estimate comparing the diameter of $W_{k}^{n}$ to the diameter of the component $W_{k-1}^{n}$ that it surrounds whenever $k \geq 1$. See Figure 10. The following is inequality (17.1) in Bis18. We include the proof to emphasize an important detail, and because it is the simplest situation that illustrates how Lemma 3.2 will be used in the next two sections. Define $R_{0}:=\operatorname{diam}\left(\Omega_{0}\right)$.

Lemma 12.1. Let $k \geq 1, \alpha \geq s$. Suppose that $W_{k-1}^{n}, W_{k}^{n}$ are both elements of $\mathcal{C}_{m}$ for some $m$, and suppose that $W_{k-1}^{n} \subset \widehat{W}_{k}^{n}$. Then $R$ may be chosen large enough so that

$$
\operatorname{diam}\left(W_{k-1}^{n}\right)^{\alpha} \leq \frac{1}{8} \operatorname{diam}\left(W_{k}^{n}\right)^{\alpha} .
$$

Proof. Observe that we have $f^{n}\left(W_{k}^{n}\right)=A_{k}$ and $f^{n}\left(W_{k-1}^{n}\right)=A_{k-1}$, and $f^{n}$ is conformal on a topological ball $B$ containing $W_{k}^{n}$. Indeed, if $n>1$, then $f^{n-1}\left(W_{k}^{n}\right)$ is contained in some petal $R_{j} \cdot \Omega_{n_{j}}^{p}$, and the proof of Lemma 8.5] says that we may take $f$ to be conformal on some ball $B^{\prime} \subset R_{j} \cdot \Omega_{n_{j}}^{p}$ which contains $f^{n-1}\left(\widehat{W}_{k}^{n}\right)$ such that the modulus of $B^{\prime} \backslash f^{n-1}\left(\widehat{W}_{k}^{n}\right)$ is bounded below by some universal constant. Then we can take $B$ to be the appropriate connected component of $\left(f^{n-1}\right)^{-1}\left(B^{\prime}\right)$. If $n=1$, we may take $B^{\prime}=B$ directly from $R_{1} \cdot \Omega_{n_{1}}^{p}$. In either case, by Lemma 3.2 there exists a constant $C$ so that

$$
\frac{\operatorname{diam}\left(W_{k-1}^{n}\right)^{\alpha}}{\operatorname{diam}\left(W_{k}^{n}\right)^{\alpha}} \leq C \frac{\operatorname{diam}\left(A_{k-1}\right)^{\alpha}}{\operatorname{diam}\left(A_{k}\right)^{\alpha}} \leq C \frac{R_{k-1}^{\alpha}}{R_{k}^{\alpha}} \leq C \frac{1}{\sqrt{R_{1}^{\alpha}}}
$$

When $R$ is sufficiently large, this proves the lemma.

Lemma 12.2 is more complicated. It says that at any stage, when we refine a component $W_{k}^{n}$ for $k \geq 1$, we can control the sum of the diameters of the refined components inside $W_{k}^{n}$ in terms of the diameter of $W_{k}^{n}$. See Figure10. This lemma corresponds to inequality (17.2) in Bis18. We will clarify the part of the proof with an estimate related to the "petal map"; the rest of the proof is the same.

Lemma 12.2. Let $k \geq 1, \alpha \geq s$. Suppose that $W_{k}^{n} \in \mathcal{C}_{m}$ with $k \geq 1$ for some $m$. Let $\widehat{W}_{k+q-1}^{n+q}$ be components of $\widehat{\mathcal{C}}_{m+1}$ contained inside of $W_{k}^{n}$. Let $W_{k}^{n}(q)$ denote the components of the form $\widehat{W}_{k+q-1}^{n+q}$ for a fixed $q \geq 1$. Then

$$
\sum_{q \geq 1} \sum_{W_{k}^{n}(q)} \operatorname{diam}\left(\widehat{W}_{k+q-1}^{n+q}\right)^{\alpha} \leq \frac{1}{8} \operatorname{diam}\left(W_{k}^{n}\right)^{\alpha} .
$$

Proof. We have $\widehat{W}_{k+q-1}^{n+q} \subset W_{k}^{n} \subset A_{1}$. Then $f^{n}\left(\widehat{W}_{k+q-1}^{n+q}\right) \subset B \subset A_{k}$, where $B$ is a ball where $f^{q}$ is conformal, constructed similarly by a pullback of some ball $B^{\prime}$ of 


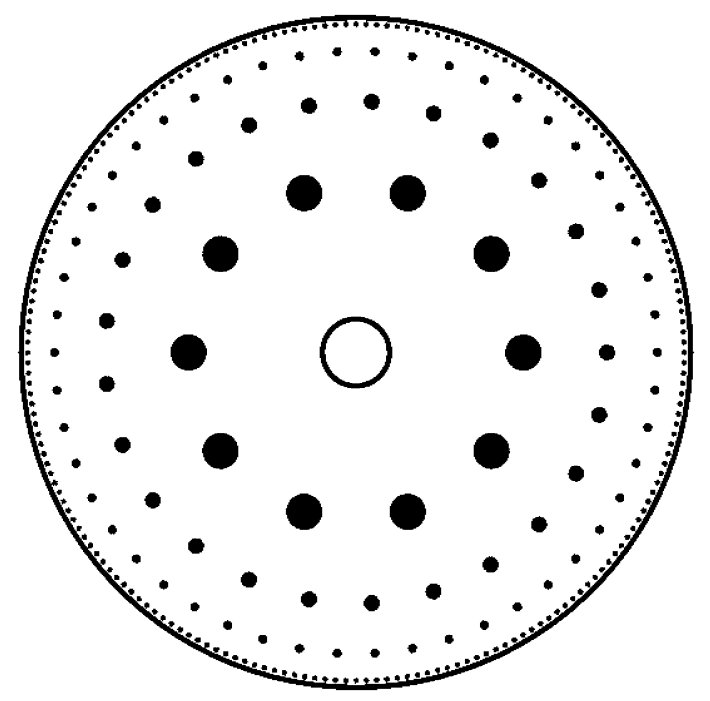

Figure 8. A schematic for Lemma 12.2. $W_{k}^{n}$ belongs to the cover$\operatorname{ing} \mathcal{C}_{m}$ and is the annulus bounded by the innermost and outermost boundary curves. The filled components correspond to elements $\widehat{W}_{k+q-1}^{n+q}$ of $\widehat{\mathcal{C}}_{m+1}$ for $q=1,2,3,4$. Lemma 12.2 says when $R$ is large enough and $k \geq 1$, the $\alpha$-sum of these components in $\widehat{\mathcal{C}}_{m+1}$ can be controlled in terms of $\operatorname{diam}\left(W_{k}^{n}\right)^{\alpha}$.

as in the proof of Lemma 12.1. By one application of Lemma 3.2 for all $q \geq 1$ we have

$$
\frac{\operatorname{diam}\left(\widehat{W}_{k+q-1}^{n+q}\right)^{\alpha}}{\operatorname{diam}\left(W_{k}^{n}\right)^{\alpha}} \leq C \frac{\operatorname{diam}\left(f^{n}\left(\widehat{W}_{k+q-1}^{n+q}\right)\right)^{\alpha}}{R_{k}^{\alpha}} .
$$

If $q=1$, then $f^{n}\left(\widehat{W}_{k}^{n+1}\right)$ belongs to a petal $R_{k} \cdot \Omega_{n_{k}}^{p}$ and $\operatorname{diam}\left(f^{n}\left(\widehat{W}_{k}^{n+1}\right)\right)=$ $O\left(R_{k}^{-2}\right)$ by Lemma 8.2. Therefore for sufficiently large $R$, (12.1) yields

$$
\frac{\operatorname{diam}\left(\widehat{W}_{k}^{n+1}\right)^{\alpha}}{\operatorname{diam}\left(W_{k}^{n}\right)^{\alpha}} \leq \frac{C}{R_{k}^{\alpha}}
$$

If $q \geq 2$, we need to be a little more careful. Another application of Lemma 3.2 with $B$ as above gives

$$
\frac{\operatorname{diam}\left(f^{n}\left(\widehat{W}_{k+q-1}^{n+q}\right)\right)^{\alpha}}{\operatorname{diam}(B)^{\alpha}} \leq C \frac{\operatorname{diam}\left(f^{n+q-1}\left(\widehat{W}_{k+q-1}^{n+q}\right)\right)^{\alpha}}{\operatorname{diam}\left(B^{\prime}\right)^{\alpha}} .
$$

Then $\operatorname{diam}\left(f^{n+q-1}\left(\widehat{W}_{k+q-1}^{n+q}\right)\right)=O\left(R_{k+q-1}^{-2}\right)$ by Lemma 8.2. If $R$ is large enough, we combine (12.2) with (12.1) to obtain

$$
\frac{\operatorname{diam}\left(\widehat{W}_{k+q-1}^{n+q}\right)^{\alpha}}{\operatorname{diam}\left(W_{k}^{n}\right)^{\alpha}} \leq \frac{C}{R_{k}^{\alpha}} .
$$

The rest of the lemma follows similarly to the proof of Lemma 16.3 on page 449 in Bis18. 


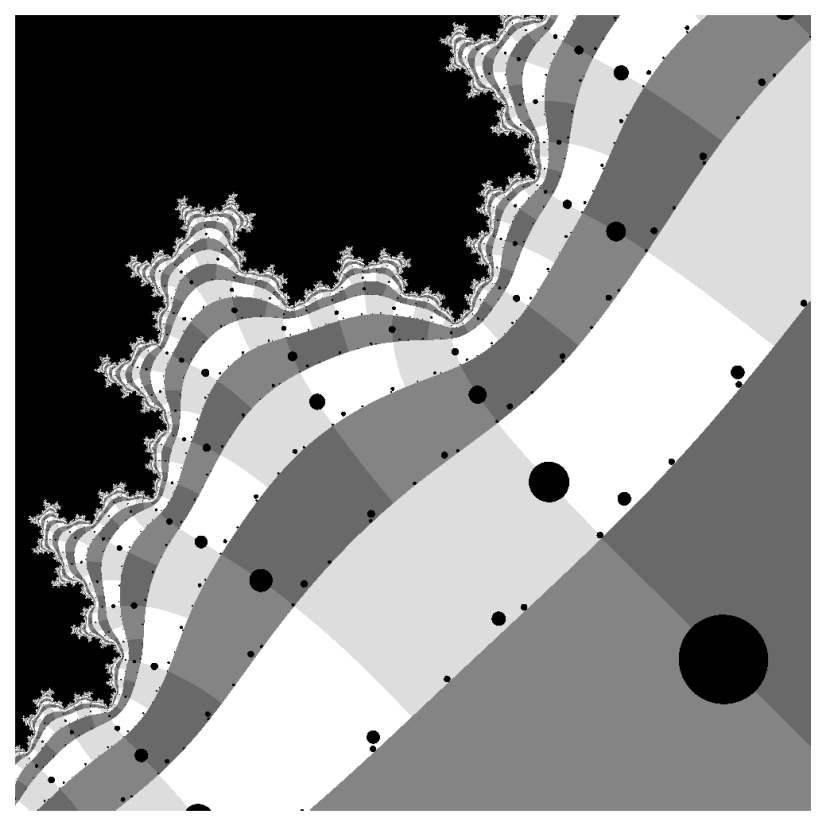

FiguRe 9. A schematic for Lemma 13.1. We see a portion of $B_{f}$, and we see annular regions representing the innermost and outermost boundaries of Fatou components $\Omega_{k}$ for $k \leq 0$. The complimentary components of $\Omega_{k}$ contained in $\Omega_{k}^{A}$ are filled in and correspond to the elements in $\mathcal{V}_{k}$. Inside each annular region, we have depicted the pieces of $\Omega_{k}$ that make up the collection $\mathcal{Q}$, which is a Whitney-type decomposition of a neighborhood of the unbounded complementary component of $J_{f}$.

\section{The $s$-Sum of the Fatou Components: REFINING NEAR $B_{f}$ AND CONCLUSIONS}

Let $\widehat{W}_{k}^{n} \in \widehat{\mathcal{C}}_{m}$ be given. Lemma 12.2 only works for components in $\widehat{\mathcal{C}}_{m+1}$ contained in the Jordan annuli $W_{j}^{n} \in \mathcal{C}_{m}$ with $W_{j}^{n} \subset \widehat{W}_{k}^{n}$ and $j \geq 1$. However, there are also components of $\mathcal{C}_{m+1}$ contained in $W_{j}^{n} \in \mathcal{C}_{m}$ with $W_{j}^{n} \subset \widehat{W}_{k}^{n}$ for $j<1$ that we must consider as well. The methods of the above two lemmas do not work as $j \rightarrow-\infty$. We handle this difficulty by using a Whitney-type decomposition.

First, we need to recall some notation from Section 11 Let $A_{1}$ be as above and $k \leq 0$. Then $A_{k}$ is the component of $\left(f^{1-k}\right)^{-1}\left(A_{1}\right)$ that surrounds the origin. Choose $z \in Y \cap A_{k}$. Then $f^{1-k}(z) \in A_{1} \cap Y$, and therefore $z \in \widehat{W}_{n}^{n} \subset A_{1}$ for some $\widehat{W}_{n}^{n} \in \widehat{\mathcal{C}}_{1}$. Then define $V_{k}^{n}$ to be the component of $\left(f^{1-k}\right)^{-1}\left(\widehat{W}_{n}^{n}\right)$ that contains $z$. Let $\mathcal{V}_{k}$ denote the set of all components $V_{k}^{n}$ contained in $A_{-k}$ obtained in this way. Let $\mathcal{V}=\bigcup_{k \leq 0} \mathcal{V}_{k}$. See Figure 9 
Lemma 13.1. Fix $\epsilon_{0}>0$, and let $\alpha \geq s+\epsilon_{0}$ and $R_{0}=\operatorname{diam}\left(\Omega_{0}\right)$. With the notation as above, there exists a constant $C$ (depending on $\epsilon_{0}$ ) so that for sufficiently large $R$,

$$
\sum_{V_{k}^{n} \in \mathcal{V}} \operatorname{diam}\left(V_{k}^{n}\right)^{\alpha} \leq C 2^{N} R_{0}^{\alpha}
$$

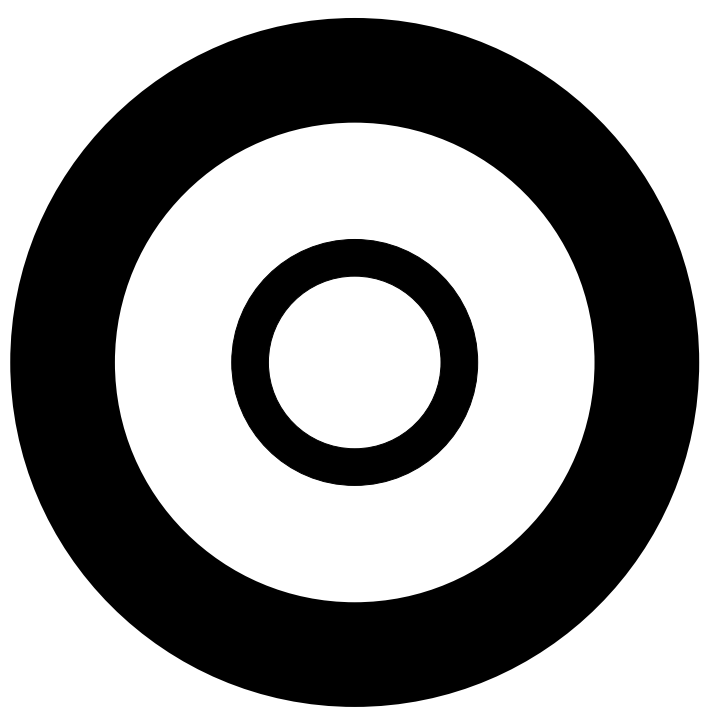

FiguRE 10. A schematic for Lemma 12.1. $W_{k}^{n}$ and $W_{k-1}^{n}$ belong to the same covering $\mathcal{C}_{m}$, and $W_{k-1}^{n}$ is contained inside of the bounded complementary component of $W_{k}^{n}$. When $R$ is large and $k \geq 1$, both components are approximately round and the diameter of $W_{k-1}^{n}$ is controlled in terms of the diameter of $W_{k}^{n}$.

Proof. For each $\Omega_{k}, k \leq 0, f: \Omega_{k}^{A} \rightarrow \Omega_{k+1}^{A}$ is a $2^{N}$-to-1 covering map, so each $\Omega_{k}^{A}$ can be decomposed into $2^{N(-k+1)}$ pieces each of which maps conformally onto $\Omega_{1}^{A}$ minus a slit, denoted as $\Omega_{1}^{S}:=\Omega_{1}^{A} \backslash \mathbb{R}^{+}$. This process breaks $\Omega_{k}^{A}$ into $2^{N(-k+1)}$ pieces and we denote this collection by $\mathcal{Q}_{k}$. Define $\mathcal{Q}=\cup_{k \leq 0} \mathcal{Q}_{k}$. We also choose to define $\mathcal{Q}$ by the dynamics of $f$, so that each $Q \in \mathcal{Q}_{k}$ maps onto some $Q^{\prime} \in \mathcal{Q}_{k+1}$. To accomplish this, it suffices to choose an appropriate decomposition of $\Omega_{0}^{A}$, and then define the decomposition of $\Omega_{k}$ for $k<0$ by inverse images, similar to Example 4.4. Also see Figure 9.

With this procedure, it is not necessarily true that each $V_{k}^{n}$ is compactly contained in some $Q \in \mathcal{Q}$. Doing the same procedure above, but with $\Omega_{1}^{A} \backslash \mathbb{R}^{-}$, we obtain a similar but "rotated" collection $i \mathcal{Q}=\bigcup_{k \leq 0} i \mathcal{Q}_{k}$. Then each $V_{k}^{n}$ is compactly contained inside at least one element of $\mathcal{Q}_{k}$ or $i \mathcal{Q}_{k}$.

We claim $\mathcal{Q}$ and $i \mathcal{Q}$ form Whitney-type decompositions of a neighborhood of the complement of the filled Julia set $K_{f}$ of the polynomial-like map $f$. Indeed, $\left(f, \widehat{\Omega}_{0}, \widehat{\Omega}_{1}\right)$ is a degree $2^{N}$ polynomial-like mapping with a connected filled Julia set and is therefore quasiconformally conjugate in $\widehat{\Omega}_{0} \backslash \overline{\mathbb{D}}$ to $z^{2^{N}}$. Moreover, elements of $\mathcal{Q}$ correspond under this conjugacy correspond to a Whitney-type decomposition 
of a neighborhood of the complement of $\overline{B(0,1)}$, as in Example 4.4. The same discussion applies to $i \mathcal{Q}$. It follows from Lemma 4.8 that $\mathcal{Q}$ and $i \mathcal{Q}$ are Whitneytype decompositions of a neighborhood of the complement of $K_{f}$.

Now, fix some $k \leq 0$ and let $Q \in \mathcal{Q}_{k}$ be given. Let $V_{k}^{n} \in \mathcal{V}_{k}$ be compactly contained in $Q$ for some $n \geq 1$. Then $f^{k+1}$ conformally maps $V_{k}^{n}$ onto some $\widehat{W}_{n}^{n} \in \widehat{\mathcal{C}}_{1}$, and by Lemma 3.2

$$
\frac{\operatorname{diam}\left(V_{k}^{n}\right)^{\alpha}}{\operatorname{diam}(Q)^{\alpha}} \leq C \frac{\operatorname{diam}\left(\widehat{W}_{n}^{n}\right)^{\alpha}}{\operatorname{diam}\left(\Omega_{1}\right)^{\alpha}} .
$$

Next, for this fixed $k \leq 0$, we sum over all components of the form $V_{k}^{n}$ for any $n \geq 1$ that are compactly contained inside of $Q$. We denote this collection by $V_{k}^{n} \subset \subset Q$. We obtain

$$
\sum_{V_{k}^{n} \subset \subset Q} \frac{\operatorname{diam}\left(V_{k}^{n}\right)^{\alpha}}{\operatorname{diam}(Q)^{\alpha}} \leq C \sum_{\widehat{W}_{n}^{n} \in \widehat{\mathcal{C}}_{1}} \frac{\operatorname{diam}\left(\widehat{W}_{n}^{n}\right)^{\alpha}}{\operatorname{diam}\left(\Omega_{1}\right)^{\alpha}} \leq \frac{C}{R_{1}^{\alpha}} \sum_{\widehat{W}_{n}^{n} \in \widehat{\mathcal{C}}_{1}} \operatorname{diam}\left(\widehat{W}_{n}^{n}\right)^{\alpha} .
$$

For this fixed $k \geq 0$, we now sum over all components of the form $V_{k}^{n}$ for any $n \geq 1$ that are compactly contained in some $Q \in \mathcal{Q}_{k}$. Then

$$
\sum_{Q \in \mathcal{Q}_{k}} \sum_{V_{k}^{n} \subset \subset Q} \operatorname{diam}\left(V_{k}^{n}\right)^{\alpha} \leq \frac{C}{R_{1}^{\alpha}} \sum_{\widehat{W}_{n}^{n} \in \widehat{\mathcal{C}}_{1}} \operatorname{diam}\left(\widehat{W}_{n}^{n}\right)^{\alpha} \cdot \sum_{Q \in \mathcal{Q}_{k}} \operatorname{diam}(Q)^{\alpha} .
$$

For this fixed $k \leq 0$, if we did the procedure above, except this time using each $Q^{\prime} \in i \mathcal{Q}_{k}$ we obtain in a similar way

$$
\sum_{Q^{\prime} \in i \mathcal{Q}_{k}} \sum_{V_{k}^{n} \subset \subset Q^{\prime}} \operatorname{diam}\left(V_{n}^{n}\right)^{\alpha} \leq \frac{C}{R_{1}^{\alpha}} \sum_{\widehat{W}_{n}^{n} \in \widehat{\mathcal{C}}_{1}} \operatorname{diam}\left(\widehat{W}_{n}^{n}\right)^{\alpha} \cdot \sum_{Q^{\prime} \in i \mathcal{Q}_{k}} \operatorname{diam}\left(Q^{\prime}\right)^{\alpha} .
$$

Note that every $V_{k}^{n}$ is compactly contained in at least one element of $\mathcal{Q}$ or $i \mathcal{Q}$. So for this fixed $k \leq 0$, we have

$$
\sum_{V_{k}^{n} \in \mathcal{V}_{k}} \operatorname{diam}\left(V_{n}^{n}\right)^{\alpha} \leq \frac{C}{R_{1}^{\alpha}}\left(\sum_{Q \in \mathcal{Q}_{k}} \operatorname{diam}(Q)^{\alpha}+\sum_{Q^{\prime} \in i \mathcal{Q}_{k}} \operatorname{diam}\left(Q^{\prime}\right)^{\alpha}\right) \sum_{\widehat{W}_{n}^{n} \in \widehat{\mathcal{C}}_{1}} \operatorname{diam}\left(\widehat{W}_{n}^{n}\right)^{\alpha} .
$$

Finally, this estimate is true for all $k \leq 0$, so summing over all such $k$ gives us

$$
\sum_{V_{k}^{n} \in \mathcal{V}} \operatorname{diam}\left(V_{k}^{n}\right)^{\alpha} \leq \frac{C}{R_{1}^{\alpha}} \sum_{\widehat{W}_{n}^{n} \in \widehat{\mathcal{C}}_{1}} \operatorname{diam}\left(\widehat{W}_{n}^{n}\right)^{\alpha} \cdot\left(\sum_{Q \in \mathcal{Q}} \operatorname{diam}(Q)^{\alpha}+\sum_{Q^{\prime} \in i \mathcal{Q}} \operatorname{diam}\left(Q^{\prime}\right)^{\alpha}\right) .
$$

Recall that $\mathcal{Q}$ and $i \mathcal{Q}$ are Whitney-type decompositions of a neighborhood of the complement of $K_{f}$. Since $\operatorname{dim}_{H}\left(J_{f}\right)=\overline{\operatorname{dim}_{M}}\left(J_{f}\right)=s$, and since $\alpha \geq s+\epsilon_{0}$, by Lemma 4.7. the $\alpha$-sum of elements of $\mathcal{Q}$ and $i \mathcal{Q}$ converges, and the $\alpha$-sum of elements in $\mathcal{Q}$ and $i \mathcal{Q}$ is comparable to the $\alpha$-sum of elements in $\mathcal{Q}_{0}$. By these observations and Lemma 12.2, for all sufficiently large $R$, we have

$$
\sum_{V_{k}^{n} \in \mathcal{V}} \operatorname{diam}\left(V_{n}^{n}\right)^{\alpha} \leq \frac{C \cdot 2^{N} R_{0}^{\alpha}}{R_{1}^{\alpha}} \cdot \sum_{\widehat{W}_{n}^{n} \in \mathcal{C}_{1}} \operatorname{diam}\left(\widehat{W}_{n}^{n}\right)^{\alpha} \leq C 2^{N} R_{0}^{\alpha}
$$

This is exactly what we wanted. 
If $\widehat{W}_{k}^{n} \in \widehat{\mathcal{C}}_{m}$, then some iterate of $f$ maps $\widehat{W}_{k}^{n}$ conformally onto $\widehat{A}_{k}$. The components of $\widehat{\mathcal{C}}_{m+1}$ that are contained inside of $W_{j}^{n} \in \mathcal{C}_{m}$ with $W_{j}^{n} \subset \widehat{W}_{k}^{n}$ for $j<1$ get conformally mapped onto the elements of $\mathcal{V}$. This allows us to prove the following more general lemma.

Lemma 13.2. Fix $\epsilon_{0}>0$ and $\alpha \geq s+\epsilon_{0}$. Consider an element of the form $\widehat{W}_{k}^{n} \in \widehat{\mathcal{C}}_{m}$ for some $m$. Let $W_{j}^{n}$ for $j \leq 1$ be the elements of $\mathcal{C}_{m}$ which are contained in $\widehat{W}_{k}^{n}$. Let $W_{j}^{n}(q)$ denote the components of the form $\widehat{W}_{j+q-1}^{n+q}$ in $\widehat{\mathcal{C}}_{m+1}$ which are contained in $W_{j}^{n}$ (we define $W_{j}^{n}(q)$ to be empty if $j+q-1 \leq 0$ ). Then there exists a sufficiently large $R$ so that the $\alpha$-sum of all the components $\widehat{W}_{j+q-1}^{n+q} \in \widehat{\mathcal{C}}_{m+1}$ contained in $W_{j}^{n}$ for $j<1$ satisfies

$$
\sum_{j \leq 0} \sum_{q=1}^{\infty} \sum_{W_{j}^{n}(q)} \operatorname{diam}\left(\widehat{W}_{j+q-1}^{n+q}\right)^{\alpha} \leq \frac{1}{8} \operatorname{diam}\left(W_{1}^{n}\right) .
$$

Proof. Choose some $\widehat{W}_{k}^{n} \in \widehat{\mathcal{C}}_{m}$. Then $f^{n}\left(\widehat{W}_{k}^{n}\right)=\widehat{A}_{k}$, and this mapping is conformal. The elements being summed in (13.1) are mapped conformally onto $\mathcal{V}$. Therefore, by Lemma 3.2 .

$$
\begin{aligned}
\sum_{j \leq 0} \sum_{q=1}^{\infty} \sum_{W_{j}^{n}(q)} \operatorname{diam}\left(\widehat{W}_{j+q-1}^{n+q}\right)^{\alpha} & \leq C \frac{\operatorname{diam}\left(W_{1}^{n}\right)^{\alpha}}{\operatorname{diam}\left(A_{1}\right)^{\alpha}} \sum_{V_{k}^{n} \in \mathcal{V}} \operatorname{diam}\left(V_{k}^{n}\right)^{\alpha} \\
& \leq \frac{C \cdot 2^{N} \cdot R_{0}^{\alpha}}{R_{1}^{\alpha}} \cdot \operatorname{diam}\left(W_{1}^{n}\right)^{\alpha} \\
& \leq \frac{1}{8} \operatorname{diam}\left(W_{1}^{n}\right)^{\alpha}
\end{aligned}
$$

whenever $R$ is large enough, as desired.

We now have everything we need to prove Theorem 10.1.

Proof of Theorem 10.1. It is sufficient to show that the $\left(s+\epsilon_{0}\right)$-sum of the elements in $\widehat{\mathcal{C}}_{m+1}$ is at most half the $\left(s+\epsilon_{0}\right)$-sum of the elements in $\widehat{\mathcal{C}}_{m}$, because then the $\left(s+\epsilon_{0}\right)$-sum in (10.1) is geometric. To accomplish this, it suffices to show that for any $\widehat{W}_{k}^{n} \in \widehat{\mathcal{C}}_{m}$, the $\left(s+\epsilon_{0}\right)$-sum of all the elements of $\widehat{\mathcal{C}}_{m+1}$ contained in $\widehat{W}_{k}^{n}$ is at most half of $\operatorname{diam}\left(W_{k}^{n}\right)^{s+\epsilon_{0}}$.

To that end, let $\widehat{W}_{k}^{n} \in \widehat{\mathcal{C}}_{m}$ be given. Using the notation of the previous lemmas, the $\left(s+\epsilon_{0}\right)$-sum of all the elements of $\widehat{\mathcal{C}}_{m+1}$ contained in $\widehat{W}_{k}^{n}$ is represented by

$$
\begin{aligned}
I=\sum_{j \leq k} \sum_{q \geq 1} \sum_{W_{j}^{n}(q)} \operatorname{diam}\left(\widehat{W}_{j+q-1}^{n+q}\right)^{s+\epsilon_{0}}= & \sum_{j=1}^{k} \sum_{q \geq 1} \sum_{W_{j}^{n}(q)} \operatorname{diam}\left(\widehat{W}_{j+q-1}^{n+q}\right)^{s+\epsilon_{0}} \\
& +\sum_{j \leq 0} \sum_{q \geq 1} \sum_{W_{j}^{n}(q)} \operatorname{diam}\left(\widehat{W}_{j+q-1}^{n+q}\right)^{s+\epsilon_{0}} .
\end{aligned}
$$

By Lemma 13.2 we have

$$
\sum_{j \leq 0} \sum_{q \geq 1} \sum_{W_{j}^{n}(q)} \operatorname{diam}\left(\widehat{W}_{j+q-1}^{n+q}\right)^{s+\epsilon_{0}} \leq \frac{1}{8} \operatorname{diam}\left(W_{1}^{n}\right)^{s+\epsilon_{0}} .
$$


Combining this with Lemma 12.2 to estimate the other sum, we have

$$
I \leq \frac{1}{4} \sum_{j=1}^{k} \operatorname{diam}\left(W_{j}^{n}\right)^{s+\epsilon_{0}} .
$$

Then repeatedly using Lemma 12.1 we can conclude that $I \leq \frac{1}{2} \operatorname{diam}\left(W_{k}^{n}\right)^{s+\epsilon_{0}}$.

Corollary 13.3. Let $\epsilon_{0}>0$. Then for sufficiently large $R$, we have $\operatorname{dim}_{\mathrm{H}}(Y) \leq$ $s+\epsilon_{0}$.

Proof. Each $\widehat{\mathcal{C}}_{m}$ is a covering of $Y \cap A_{1}$. The proof of Theorem 10.1 shows that the $\left(s+\epsilon_{0}\right)$-sum of all components of all $\widehat{\mathcal{C}}_{m}$ 's converges, and therefore the $\left(s+\epsilon_{0}\right)$-sum of the elements in $\widehat{\mathcal{C}}_{m}$ tends to 0 as $m \rightarrow \infty$. Therefore $\operatorname{dim}_{\mathrm{H}}\left(Y \cap A_{1}\right) \leq s+\epsilon_{0}$. The same arguments in these sections can be modified to show that $\operatorname{dim}_{\mathrm{H}}\left(Y \cap A_{k}\right) \leq s+\epsilon_{0}$ for all $k \in \mathbb{Z}$. Therefore $\operatorname{dim}_{\mathrm{H}}(Y) \leq s+\epsilon_{0}$.

\section{Estimating the PACKING Dimension}

In this section, we prove that the packing dimension of $\mathcal{J}(f)$ can be taken to be arbitrarily close to its Hausdorff dimension. We do this by estimating the local upper Minkowski dimension using Theorem 4.7. To do this, we will need to show the $\left(s+\epsilon_{0}\right)$-sum of a Whitney-type decomposition of the complement of the Julia set contained in the neighborhood $\Omega_{1}^{A}$ is finite. This will require separating the components into wiggly, round, and basin components, and performing some useful decompositions of these components.

The following result follows from the results of Sullivan in Sul83. (see Theorems 3 and 4$)$. Recall that if $f$ is polynomial-like, $K_{f}$ denotes its filled Julia set.

Theorem 14.1. Let $f: U \rightarrow V$ be a hyperbolic polynomial-like map. Then we have $\operatorname{dim}_{\mathrm{P}}\left(\partial K_{f}\right)=\operatorname{dim}_{\mathrm{H}}\left(\partial K_{f}\right)=\overline{\operatorname{dim}_{\mathrm{M}}}\left(\partial K_{f}\right)$.

In particular, this result applies to $f$ when viewed as a polynomial-like map. So this result applies to $J_{f}$, the quasicircle Julia set of $f$.

In order to apply Lemma 4.7, we need to decompose the Fatou components of $f$ into simpler pieces. First we collect the following lemmas proved in Section 20 of Bis18. The first lemma will allow us to break the infinitely connected Fatou components into simpler, annular regions and still conclude the convergence of a $t$-sum of a Whitney-type decomposition.

Lemma 14.2. Let $\Omega$ be a bounded open set containing disjoint open subsets $\left\{\Omega_{j}\right\}_{j=1}^{\infty}$ so that $\Omega \backslash \bigcup_{j=1}^{\infty} \Omega_{j}$ has zero Lebesgue measure. Let $W(\Omega)$ be a Whitney-type decomposition of $\Omega$ and for each $j \geq 1$, let $W\left(\Omega_{j}\right)$ be a Whitney-type decomposition for $\Omega_{j}$. Then for any $t \in(1,2]$ we have

$$
\sum_{Q \in W(\Omega)} \operatorname{diam}(Q)^{t} \leq \sum_{j=1}^{\infty} \sum_{Q \in W\left(\Omega_{j}\right)} \operatorname{diam}(Q)^{t} .
$$

Figure 11 illustrates how Lemma 14.2 will be implemented. By Theorem 10.9. the complementary components of the Fatou component $\Omega_{1}$ contained in $\Omega_{1}^{A}$ are arranged in approximately circular rings, which can be connected by an approximate circle. Doing this for every ring of complementary components, we obtain a countable union of Jordan annuli. This can be done for every Fatou component $\omega \subset \Omega_{1}^{A}$. We call this procedure necklacing the Fatou component. 


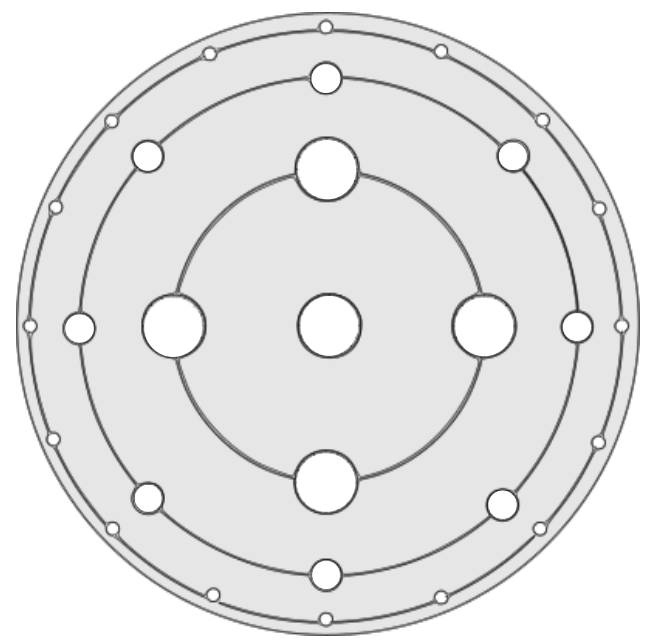

Figure 11. A schematic for the necklacing construction. Holes of $\Omega_{k}$ in the same circular ring are connected via approximate circles, and this construction can be repeated for all $\omega \subset \Omega_{k}^{A}$ by pulling back this construction. The result is the multiply connected Fatou components are now decomposed into topological annuli, which can be straightened into round annuli by a biLipshitz map. Lemmas 14.2 says that it suffices to estimate the critical exponent for a Whitney-type decomposition of the complement of the "necklaced" Julia set of $f$.

Lemma 14.3. Suppose $f: \Omega_{1} \rightarrow \Omega_{2}$ is L-biLipschitz, and let $W\left(\Omega_{1}\right)$ and $W\left(\Omega_{2}\right)$ be Whitney-type decompositions for $\Omega_{1}$ and $\Omega_{2}$. Then for any $t \in(0,2]$, there is a constant $C$ depending only on $L$ and the constants defining the Whitney-type decompositions so that we have

$$
\frac{1}{C} \sum_{Q^{\prime} \in W\left(\Omega_{2}\right)} \operatorname{diam}\left(Q^{\prime}\right)^{t} \leq \sum_{Q \in W\left(\Omega_{1}\right)} \operatorname{diam}(Q)^{t} \leq C \sum_{Q^{\prime} \in W\left(\Omega_{2}\right)} \operatorname{diam}\left(Q^{\prime}\right)^{t} .
$$

Proof. The image of $W\left(\Omega_{1}\right)$ under $f$ is a Whitney-type decomposition by Lemma 4.8, indeed, $L$-BiLipschitz maps are always $L^{2}$-quasiconformal. So by Corollary 4.10, the $t$-sums of $W\left(f\left(\Omega_{1}\right)\right)$ and $W\left(\Omega_{2}\right)$ are comparable depending on $L$ and the constants defining the Whitney-type decompositions. If $Q \in W\left(\Omega_{1}\right)$ then by the biLipschitz condition, $L^{-1} \operatorname{diam}(Q) \leq \operatorname{diam}(f(Q)) \leq L \operatorname{diam}(Q)$. Therefore the $t$-sums of $W\left(f\left(\Omega_{1}\right)\right)$ and $W\left(\Omega_{1}\right)$ are comparable depending on $L$, and the result follows.

We will use Lemma 14.3 to map the decomposed round components onto round annuli, where we can estimate the $t$-sum directly.

Lemma 14.4. Let $A=A(\rho, \rho(1+\delta))=\{z: \rho \leq|z| \leq \rho(1+\delta)\}$ be a round annulus with $\delta, \rho>0$. Let $W(A)$ denote a Whitney-type decomposition of $A$. Then for $t>1$,

$$
\sum_{Q \in W(A)} \operatorname{diam}(Q)^{t} \leq O\left(\frac{1}{1-2^{t-1}} \delta^{t-1} \rho^{t}\right)
$$


Proof. We first construct a suitable Whitney-type decomposition of $A(1,1+\delta)$; see Figure 12, The result will follow from the observations in Section 4 and applying the map $z \mapsto \rho z$. For the given $\delta>0$ there exists $N$ so that $\delta \in\left[2^{-N}, 2^{-N+1}\right)$. Let

$$
S=\left\{z=r e^{2 \pi i \theta}: r \in(1+\delta / 4,1+3 \delta / 4), \theta \in\left(0,2^{-N}\right)\right\} .
$$

$\operatorname{Both} \operatorname{diam}(S)$ and $\operatorname{dist}(S, \partial A)$ are comparable to $2^{-N}$. Then rotating $S$ exactly $2^{N}$ many times gives the first layer of the Whitney-type decomposition which we denote by $\mathcal{S}_{1}$. To get subsequent layers, we define

$$
\begin{gathered}
S_{1}=\left\{z=r e^{i \theta}: R \in(R+\delta / 8, R+\delta / 4), \theta \in\left(0,2^{-N-1}\right)\right\} . \\
S_{1}^{\prime}=\left\{z=r e^{2 \pi i \theta}: R \in(R+3 \delta / 4, R+7 \delta / 8), \theta \in\left(0,2^{-N-1}\right)\right\} .
\end{gathered}
$$

Then $\operatorname{diam}\left(S_{1}\right)$ and $\operatorname{dist}\left(S_{1}, \partial A\right)$ are both comparable to $2^{-N-1}$, and the same is true for $S_{1}^{\prime}$. Then rotating $S_{1}$ and $S_{1}^{\prime} 2^{N+1}$ many times generates $\mathcal{S}_{2}$, the second layer of the Whitney-type decomposition. Proceeding inductively, we obtain a Whitney-type decomposition $W(A)$.

The $t$-sum for the elements in $\mathcal{S}_{1}$ is comparable to $2^{N(1-t)}$, and the $t$-sum for the elements in $\mathcal{S}_{n+1}$ is comparable to $2^{(N+n)(1-t)}$. Therefore, the $t$-sum of $W(A)$ is geometric and comparable to the quantity

$$
\frac{2^{N(1-t)}}{1-2^{1-t}}
$$

$2^{N}$ and $1 / \delta$ are both comparable so we obtain

$$
\sum_{Q \in W(A)} \operatorname{diam}(Q)^{t}=O\left(\frac{1}{1-2^{1-t}} \delta_{j}^{t-1}\right),
$$

as desired.

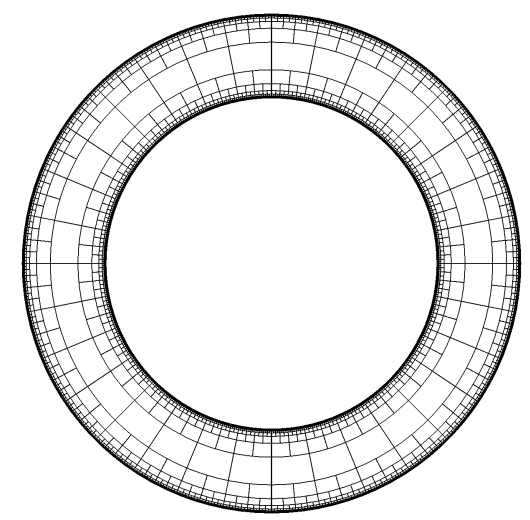

Figure 12. An illustration of a Whitney-type decomposition of the annulus described in Lemma 14.4

The following is the fundamental estimate for round Fatou components. See Theorem 20.3 in [Bis18]; we sketch the proof below. 
Theorem 14.5. $W\left(\omega_{k}\right)$ be a Whitney-type decomposition for a Fatou component $\omega_{k}$ of $k$-type, $k \geq 1$, and let $t \geq s+\epsilon_{0}$. Then

$$
\sum_{Q \in W\left(\omega_{k}\right)} \operatorname{diam}(Q)^{t}=O\left(\frac{1}{1-2^{1-t}} \operatorname{diam}\left(\omega_{k}\right)^{t}\right) .
$$

Proof. First, perform the necklacing decomposition on $\omega_{k}$, decomposing $\omega_{k}$ into countably many Jordan annuli. By Lemma 14.2, it suffices to estimate the $t$-sum of a Whitney-type decomposition of all of these Jordan annuli. By Lemma 10.11 and Lemma 3.2, these annuli are biLipschitz equivalent to round annuli $A\left(r, r\left(1+\delta_{j}\right)\right)$, where $r$ is the diameter of $\omega_{k}$, and $\left\{\delta_{j}\right\}_{j=1}^{\infty}$ tends superexponentially to 0 . Therefore, by Lemmas 14.3 and 14.4 , we have,

$\sum_{Q \in W\left(\omega_{k}\right)} \operatorname{diam}(Q)^{t}=O\left(\frac{1}{1-2^{1-t}} \sum_{j=1}^{\infty} \delta_{j}^{t-1} \operatorname{diam}\left(\omega_{k}\right)^{t}\right)=O\left(\frac{1}{1-2^{1-t}} \operatorname{diam}\left(\omega_{k}\right)^{t}\right)$, as desired.

Next we show how to control the critical exponent for the boundaries of the central series of Fatou components.

Lemma 14.6. Fix some $n \geq 1$. Let $W(\mathcal{J}(f))$ be a Whitney-type decomposition of the complement of $\mathcal{J}(f)$, and let $W$ denote the elements of $W(\mathcal{J}(f))$ contained in $B_{f} \cup\left(\cup_{k \leq n} \Omega_{k}\right)$. Then

$$
\sum_{Q \in W} \operatorname{diam}(Q)^{s+\epsilon_{0}} \leq M_{n}<\infty
$$

Proof. We split the $\left(s+\epsilon_{0}\right)$-sum into two pieces

$$
\sum_{Q \in W} \operatorname{diam}(Q)^{s+\epsilon_{0}}=I+I I+I I I .
$$

$I$ is the sum of the $Q \in W$ contained in $B_{f}, I I$ is the sum of the $Q \in W$ contained in round $\Omega_{k}$, and $I I I$ is the sum of the $Q \in W$ contained in wiggly $\Omega_{k}$.

$I$ converges by definition of the critical exponent. The $Q \in W$ which satisfy $Q \in$ $B_{f}$ form a Whitney-type decomposition of the bounded complementary component of $J_{f}$. Since $\overline{\operatorname{dim}_{\mathrm{M}}}\left(J_{f}\right)=s, I$ converges.

II converges by Theorem 14.5 .

III requires some work. The necklacing construction pulls back to $\Omega_{k}$ for $k \leq 0$. Let $\mathcal{Q}=\bigcup_{k \leq 0} \mathcal{Q}_{k}$ be the collection of pieces from Lemma 13.1. The boundaries of the elements $Q \in \mathcal{Q}$ further decompose the necklaced versions of $\Omega_{k}$ into necklaced quadrilateral-type pieces. By Lemma 14.2, it is sufficient to estimate the $\left(s+\epsilon_{0}\right)$ sum $I I I$ by estimating a $\left(s+\epsilon_{0}\right)$-sum for a Whitney-type decomposition $W$ of the complement of all of the necklaced quadrilateral-type pieces of $\Omega_{k}$.

Choose some $Q \in \mathcal{Q}_{k}$ (note that $k \leq 0$ ). Lemma 3.2 implies that for all $S \in W$, $S \subset Q$, we have

$$
\frac{\operatorname{diam}(S)^{s+\epsilon_{0}}}{\operatorname{diam}(Q)^{s+\epsilon_{0}}} \leq C \frac{\operatorname{diam}\left(f^{-k+1}(S)\right)^{s+\epsilon_{0}}}{\operatorname{diam}\left(\Omega_{1}\right)^{s+\epsilon_{0}}} .
$$

Since $f$ is conformal on $Q$, the Whitney-type decomposition for the necklaced pieces of $\Omega_{k}$ get mapped to a Whitney-type decomposition for the necklaced $\Omega_{1}^{S}$, where $\Omega_{1}^{S}=\Omega_{1} \backslash \mathbb{R}^{\geq 0}$ is the slit version of $\Omega_{1}$. Similar to the proofs of Theorem 14.5 and Theorem 14.4 the $\left(s+\epsilon_{0}\right)$-sum of a Whitney-type decomposition for the necklaced 
$\Omega_{1}^{S}$ is finite and comparable to $\operatorname{diam}\left(\Omega_{1}\right)^{s+\epsilon_{0}}$. Summing over all $S \subset Q$ and applying Corollary 4.10, we have

$$
\sum_{S \in W, S \subset Q} \operatorname{diam}(S)^{s+\epsilon_{0}} \leq \frac{C}{R_{1}^{s+\epsilon_{0}}} \operatorname{diam}(Q)^{s+\epsilon_{0}} .
$$

Therefore, when summing over all $S \in W$, we sum over all $Q \in \mathcal{Q}$ and obtain

$$
I I I \leq \sum_{S \in W} \operatorname{diam}(S)^{s+\epsilon_{0}} \leq \frac{C}{R_{1}^{s+\epsilon_{0}}} \sum_{Q \in \mathcal{Q}} \operatorname{diam}(Q)^{s+\epsilon_{0}}<\infty,
$$

where the sum converges by Lemma 4.7 .

Let $W\left(\Omega_{1}^{A}\right)$ denote a Whitney-type decomposition for $\Omega_{1}^{A} \backslash \mathcal{J}(f)$.

Theorem 14.7. The $\left(s+\epsilon_{0}\right)$-sum of the Whitney-type decomposition $W\left(\Omega_{1}^{A}\right)$ converges.

Proof. Similar to Lemma 14.6, the Fatou components have three types, and we decompose the $\left(s+\epsilon_{0}\right)$-sum into three pieces. We write

$$
\sum_{Q \subset W\left(\Omega_{1}^{A}\right)} \operatorname{diam}(Q)^{s+\epsilon_{0}}=I+I I+I I I .
$$

Recalling Definition 10.7, $I$ represents the $\left(s+\epsilon_{0}\right)$-sum in basin Fatou components, $I I$ represents the $\left(s+\epsilon_{0}\right)$-sum in round components, and $I I I$ represents the $\left(s+\epsilon_{0}\right)$ sum in wiggly components.

First we estimate $I$. Let $B_{f}^{\prime} \subset \Omega_{1}^{A}$ be a basin. Then there exists a positive integer $m$ and a unique Fatou component $\omega_{1}$ of 1 -type so that $B_{f}^{\prime} \subset \widehat{\omega}_{1}$ and $f^{m}: \widehat{\omega}_{1} \rightarrow \widehat{\Omega}_{1}$ is conformal. So for all $Q \subset B_{f}^{\prime}$, by Lemma 3.2

$$
\sum_{Q \in B_{f}^{\prime}} \operatorname{diam}(Q)^{s+\epsilon_{0}} \leq C \operatorname{diam}\left(\omega_{1}\right)^{s+\epsilon_{0}} \sum_{f^{m}(Q) \in B_{f}} \operatorname{diam}\left(f^{m}(Q)\right)^{s+\epsilon_{0}} .
$$

By Corollary 4.10, the sum above is comparable to an $\left(s+\epsilon_{0}\right)$-sum of a fixed Whitney-type decomposition for $B_{f}$, which we discussed converges in the proof of Lemma 14.6. Therefore, by summing over every basin $B_{f}^{\prime} \subset \Omega_{1}$, we can apply Theorem 10.1 and conclude that

$$
I \leq C \sum_{\omega_{1} \subset \Omega_{1}^{A}} \operatorname{diam}\left(\omega_{1}\right)^{s+\epsilon_{0}}<\infty .
$$

II converges by Theorem [14.5] and Theorem 10.1 .

Finally we estimate $I I I$. For every component $\omega$ of 1-type contained in $\Omega_{1}^{A}, \widehat{\omega}$ contains a unique sequence of components $\omega_{k}$ of $k$-type for $k \leq 0$ so that there exists a positive integer $m$ so that $f^{m}(\omega)=\Omega_{1}, f^{m}\left(\omega_{k}\right)=\Omega_{k}$, and $f^{m}$ is conformal on $\widehat{\omega}$. Let $\omega$ be of 1-type and $\omega_{k}$ be its associated sequence of wiggly components. By Lemma 14.6, for some fixed choice of Whitney-type decomposition, the convergent sum in (14.2) is bounded above by some constant $M_{1}$, defined in (14.1). Therefore, we may apply Corollary 4.10 and Lemma 3.2 to conclude that

$$
\sum_{k \leq 0} \sum_{Q \subset \omega_{k}} \operatorname{diam}(Q)^{s+\epsilon_{0}} \leq C \cdot M_{1} \cdot \operatorname{diam}(\omega)^{s+\epsilon_{0}} .
$$

Again, Theorem 10.1 allows us to use (14.3) to conclude that $I I I<\infty$.

Corollary 14.8. The packing dimension of $\mathcal{J}(f)$ is at most $s+\epsilon_{0}$. 
Proof. By Theorem 14.7 the critical exponent for $\mathcal{J}(f) \cap \Omega_{1}^{A}$ is less than or equal to $s+\epsilon_{0}$. Since $\mathcal{J}(f)$ has zero Lebesgue measure, Theorem 4.7 says that the upper Minkowski dimension of $\mathcal{J}(f) \cap \Omega_{1}^{A}$ is also less than or equal to $s+\epsilon_{0}$. By Theorem 1.2 of RS05, since $f$ has no exceptional values, the upper Minkowski dimension of $\mathcal{J}(f) \cap \Omega_{1}^{A}$ coincides with the packing dimension of $\mathcal{J}(f)$, so that $\operatorname{dim}_{\mathrm{P}}(\mathcal{J}(f)) \leq s+\epsilon_{0}$.

\section{ACKNOWLEDGMENTS}

The author would like to thank Chris Bishop for suggesting this problem, offering many useful suggestions, and for reading and offering detailed feedback on earlier drafts. David Sixsmith found many mistakes and typos and offered suggestions that greatly improved the exposition of this paper. The author would also like to thank the referee for detailed and helpful reports that found many places to improve this paper, and would also like to recognize Misha Lyubich, Lasse Rempe-Gillen, Gwyneth Stallard, and Phil Rippon for helpful discussions.

\section{REFERENCES}

[Bak75] I. N. Baker, The domains of normality of an entire function, Ann. Acad. Sci. Fenn. Ser. A I Math. 1 (1975), no. 2, 277-283. MR0402044

[Bis18] Christopher J. Bishop, A transcendental Julia set of dimension 1, Invent. Math. 212 (2018), no. 2, 407-460, DOI 10.1007/s00222-017-0770-0. MR3787831

[BP17] Christopher J. Bishop and Yuval Peres, Fractals in probability and analysis, Cambridge Studies in Advanced Mathematics, vol. 162, Cambridge University Press, Cambridge, 2017, DOI 10.1017/9781316460238. MR3616046

[BR86] Lipman Bers and H. L. Royden, Holomorphic families of injections, Acta Math. 157 (1986), no. 3-4, 259-286, DOI 10.1007/BF02392595. MR857675

[BRS13] Walter Bergweiler, Philip J. Rippon, and Gwyneth M. Stallard, Multiply connected wandering domains of entire functions, Proc. Lond. Math. Soc. (3) 107 (2013), no. 6, 1261-1301, DOI 10.1112/plms/pdt010. MR.3149847

[CG93] Lennart Carleson and Theodore W. Gamelin, Complex dynamics, Universitext: Tracts in Mathematics, Springer-Verlag, New York, 1993, DOI 10.1007/978-1-4612-4364-9. MR.1230383

[DH85] Adrien Douady and John Hamal Hubbard, On the dynamics of polynomial-like mappings, Ann. Sci. École Norm. Sup. (4) 18 (1985), no. 2, 287-343. MR816367

[Dom97] P. Domínguez, Connectedness properties of Julia sets of transcendental entire functions, Complex Variables Theory Appl. 32 (1997), no. 3, 199-215, DOI 10.1080/17476939708814991. MR 1457686

[GM05] John B. Garnett and Donald E. Marshall, Harmonic measure, New Mathematical Monographs, vol. 2, Cambridge University Press, Cambridge, 2005, DOI 10.1017/CBO9780511546617. MR 2150803

[Hei01] Juha Heinonen, Lectures on analysis on metric spaces, Universitext, Springer-Verlag, New York, 2001, DOI 10.1007/978-1-4613-0131-8. MR.1800917

[McM87] Curt McMullen, Area and Hausdorff dimension of Julia sets of entire functions, Trans. Amer. Math. Soc. 300 (1987), no. 1, 329-342, DOI 10.2307/2000602. MR871679

[McM94] Curtis T. McMullen, Complex dynamics and renormalization, Annals of Mathematics Studies, vol. 135, Princeton University Press, Princeton, NJ, 1994. MR1312365

[Mis81] Michał Misiurewicz, On iterates of $e^{z}$, Ergodic Theory Dynam. Systems 1 (1981), no. 1, 103-106, DOI 10.1017/s014338570000119x. MR627790

[PU10] Feliks Przytycki and Mariusz Urbański, Conformal fractals: ergodic theory methods, London Mathematical Society Lecture Note Series, vol. 371, Cambridge University Press, Cambridge, 2010, DOI 10.1017/CBO9781139193184. MR2656475

[RGS17] Lasse Rempe-Gillen and Dave Sixsmith, Hyperbolic entire functions and the EremenkoLyubich class: class $\mathcal{B}$ or not class $\mathcal{B}$ ?, Math. Z. 286 (2017), no. 3-4, 783-800, DOI 10.1007/s00209-016-1784-9. MR.3671560 
[RS05] P. J. Rippon and G. M. Stallard, Dimensions of Julia sets of meromorphic functions, J. London Math. Soc. (2) 71 (2005), no. 3, 669-683, DOI 10.1112/S0024610705006393. MR2132377

[RS12] P. J. Rippon and G. M. Stallard, Fast escaping points of entire functions, Proc. Lond. Math. Soc. (3) 105 (2012), no. 4, 787-820, DOI 10.1112/plms/pds001. MR2989804

[Sch10] Dierk Schleicher, Dynamics of entire functions, Holomorphic dynamical systems, Lecture Notes in Math., vol. 1998, Springer, Berlin, 2010, pp. 295-339, DOI 10.1007/9783-642-13171-4_5. MR2648691

[Shi98] Mitsuhiro Shishikura, The Hausdorff dimension of the boundary of the Mandelbrot set and Julia sets, Ann. of Math. (2) 147 (1998), no. 2, 225-267, DOI 10.2307/121009. MR.1626737

[Sta91] Gwyneth M. Stallard, The Hausdorff dimension of Julia sets of entire functions, Ergodic Theory Dynam. Systems 11 (1991), no. 4, 769-777, DOI 10.1017/S0143385700006477. MR 1145621

[Sta96] Gwyneth M. Stallard, The Hausdorff dimension of Julia sets of entire functions. II, Math. Proc. Cambridge Philos. Soc. 119 (1996), no. 3, 513-536, DOI 10.1017/S0305004100074387. MR1357062

[Sta97] Gwyneth M. Stallard, The Hausdorff dimension of Julia sets of entire functions. III, Math. Proc. Cambridge Philos. Soc. 122 (1997), no. 2, 223-244, DOI 10.1017/S0305004197001722. MR1458228

[Sta00] Gwyneth M. Stallard, The Hausdorff dimension of Julia sets of entire functions. IV, J. London Math. Soc. (2) 61 (2000), no. 2, 471-488, DOI 10.1112/S0024610799008601. MR:1760674

[Sta08] Gwyneth M. Stallard, Dimensions of Julia sets of transcendental meromorphic functions, Transcendental dynamics and complex analysis, London Math. Soc. Lecture Note Ser., vol. 348, Cambridge Univ. Press, Cambridge, 2008, pp. 425-446, DOI 10.1017/CBO9780511735233.017. MR2458811

[Ste70] Elias M. Stein, Singular integrals and differentiability properties of functions, Princeton Mathematical Series, No. 30, Princeton University Press, Princeton, N.J., 1970. MR0290095

[Sul83] Dennis Sullivan, Conformal dynamical systems, Geometric dynamics (Rio de Janeiro, 1981), Lecture Notes in Math., vol. 1007, Springer, Berlin, 1983, pp. 725-752, DOI 10.1007/BFb0061443. MR730296

[Var11] Dror Varolin, Riemann surfaces by way of complex analytic geometry, Graduate Studies in Mathematics, vol. 125, American Mathematical Society, Providence, RI, 2011, DOI 10.1090/gsm/125. MR2798295

[Zhe06] Jian-Hua Zheng, On multiply-connected Fatou components in iteration of meromorphic functions, J. Math. Anal. Appl. 313 (2006), no. 1, 24-37, DOI 10.1016/j.jmaa.2005.05.038. MR2178719

Department of Mathematics, University of Wisconsin, 480 Lincoln Drive, Madison, WisCONSIN 53706

Email address: burkart2@wisc.edu 